\title{
MARC Reborn: Migrating MARC Fixed Field Metadata into the Variable Fields
}

\section{Appendix}

Conversion Table for Map

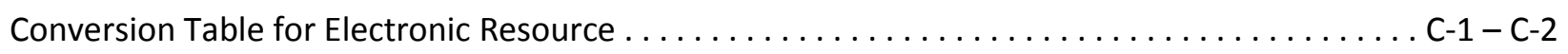

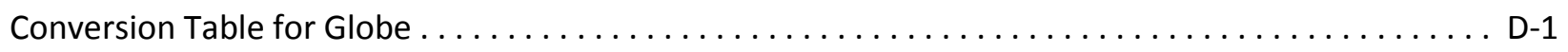

Conversion Table for Tactile Material $\ldots \ldots \ldots \ldots \ldots \ldots \ldots \ldots \ldots \ldots \ldots \ldots \ldots \ldots \ldots \ldots \ldots \ldots \ldots \ldots \ldots \ldots$

Conversion Table for Projected Graphic $\ldots \ldots \ldots \ldots \ldots \ldots \ldots \ldots \ldots \ldots \ldots \ldots \ldots \ldots \ldots \ldots \ldots \ldots \ldots \ldots \ldots$

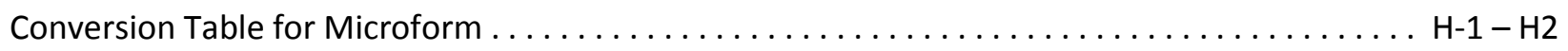

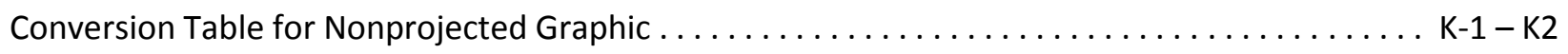

Conversion Table for Motion Picture $\ldots \ldots \ldots \ldots \ldots \ldots \ldots \ldots \ldots \ldots \ldots \ldots \ldots \ldots \ldots \ldots \ldots \ldots \ldots$

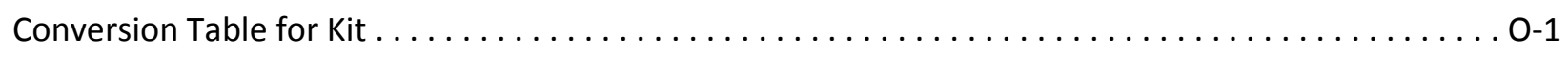

Conversion Table for Notated Music $\ldots \ldots \ldots \ldots \ldots \ldots \ldots \ldots \ldots \ldots \ldots \ldots \ldots \ldots \ldots \ldots \ldots \ldots \ldots \ldots \ldots \ldots \ldots$

Conversion Table for Remote-sensing Image $\ldots \ldots \ldots \ldots \ldots \ldots \ldots \ldots \ldots \ldots \ldots \ldots \ldots \ldots \ldots \ldots \ldots$

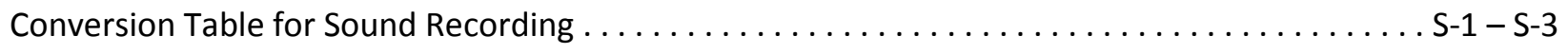

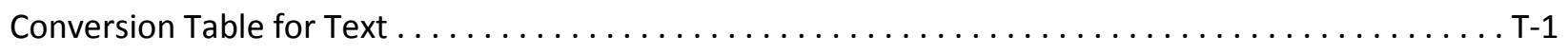

Conversion Table for Videorecording $\ldots \ldots \ldots \ldots \ldots \ldots \ldots \ldots \ldots \ldots \ldots \ldots \ldots \ldots \ldots \ldots \ldots \ldots \ldots \ldots \ldots$

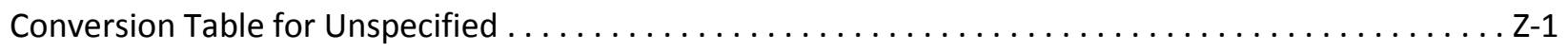

Conversion Table for the Fixed Field $\ldots \ldots \ldots \ldots \ldots \ldots \ldots \ldots \ldots \ldots \ldots \ldots \ldots \ldots \ldots \ldots \ldots \ldots \ldots \ldots \ldots \ldots$ 
MARC Reborn: Migrating MARC Fixed Field Metadata into the Variable Fields

Conversion Table for Map (007 $\ddagger a=a)$

\begin{tabular}{|c|c|c|c|c|}
\hline 007 Subfield & Value & Variable Field & Value & Notes \\
\hline$b$ & $d$ & $300 \neq f$ & atlas & \\
\hline$b$ & g & $300 \ddagger f$ & diagram & \\
\hline b & j & $300 \neq f$ & map & \\
\hline$b$ & k & $300 \ddagger f$ & profile & \\
\hline$b$ & $q$ & $300 \neq f$ & model & \\
\hline$b$ & $r$ & $300 \ddagger f$ & remote-sensing image & \\
\hline$b$ & s & $300 \neq f$ & section & \\
\hline$b$ & $\mathrm{u}$ & $300 \ddagger f$ & unspecified & \\
\hline$b$ & y & $300 \neq f$ & view & \\
\hline $\mathrm{b}$ & z & $300 \neq f$ & other & \\
\hline$d$ & a & $300 \neq b$ & black and white & Concatenation required \\
\hline d & $\mathrm{c}$ & $300 \ddagger b$ & color & Concatenation required \\
\hline$\overline{\mathrm{e}}$ & $a$ & $340 \neq a$ & paper & \\
\hline e & $\mathrm{b}$ & $340 \neq a$ & wood & \\
\hline e & c & $340 \neq a$ & stone & \\
\hline e & $d$ & $340 \neq a$ & metal & \\
\hline e & e & $340 \neq a$ & synthetic & \\
\hline e & $f$ & $340 \neq a$ & skin & \\
\hline e & g & $340 \neq a$ & textile & \\
\hline e & $\mathrm{i}$ & $340 \neq a$ & plastic & \\
\hline e & j & $340 \neq a$ & glass & \\
\hline e & 1 & $340 \neq a$ & vinyl & \\
\hline e & $\mathrm{n}$ & $340 \neq a$ & vellum & \\
\hline e & $\mathrm{p}$ & $340 \neq a$ & plaster & \\
\hline e & $q$ & $340 \neq a$ & flexible base photographic medium, positive & Proposed RDA value \\
\hline e & r & $340 \neq a$ & flexible base photographic medium, negative & Proposed RDA value \\
\hline e & $\mathrm{s}$ & $340 \neq a$ & non-flexible base photographic medium, positive & Proposed RDA value \\
\hline e & $\mathrm{t}$ & $340 \ddagger a$ & non-flexible base photographic medium, negative & Proposed RDA value \\
\hline e & u & & & No equivalent value \\
\hline e & $\mathrm{v}$ & $340 \ddagger a$ & leather & \\
\hline e & w & $340 \neq a$ & parchment & \\
\hline e & y & $340 \ddagger a$ & & No equivalent value \\
\hline $\mathrm{e}$ & z & $340 \neq a$ & & No equivalent value \\
\hline$f$ & $f$ & $340 \neq j$ & facsimile & Proposed RDA value \\
\hline f & $\mathrm{n}$ & & & No equivalent value \\
\hline f & $\mathrm{u}$ & & & No equivalent value \\
\hline f & z & & & No equivalent value \\
\hline g & a & $340 \neq d$ & photocopy, blueline & \\
\hline g & $\mathrm{b}$ & $340 \neq d$ & photocopy & \\
\hline g & c & $340 \neq d$ & pre-production & Proposed RDA value \\
\hline g & $d$ & $340 \neq d$ & film & Proposed RDA value \\
\hline g & u & & & No equivalent value \\
\hline g & $z$ & & & No equivalent value \\
\hline $\mathrm{h}$ & $a$ & $340 \ddagger 0$ & positive & \\
\hline $\mathrm{h}$ & $b$ & 340 ‡о & negative & \\
\hline $\mathrm{h}$ & $\mathrm{m}$ & 340 ‡o & mixed polarity & \\
\hline $\mathrm{h}$ & $\mathrm{n}$ & & & No equivalent value \\
\hline
\end{tabular}


Conversion Table for Electronic Resource (007 $¥ \mathrm{a}=\mathrm{c}$ )

\begin{tabular}{|c|c|c|c|c|}
\hline 007 Subfield & Value & Variable Field & Value & Notes \\
\hline $\mathrm{b}$ & $\mathrm{a}$ & $338 \neq a$ & computer tape cartridge & \\
\hline $\mathrm{b}$ & $b$ & $338 \neq a$ & computer chip cartridge & \\
\hline $\mathrm{b}$ & c & $338 \neq a$ & computer disc cartridge & \\
\hline $\mathrm{b}$ & $d$ & $338 \neq a$ & computer disc & \\
\hline$b$ & $\mathrm{e}$ & $338 \neq a$ & computer disc cartridge & \\
\hline$b$ & $f$ & $338 \neq a$ & computer tape cassette & \\
\hline$b$ & $\mathrm{~h}$ & $338 \neq a$ & computer tape reel & \\
\hline $\mathrm{b}$ & j & $338 \neq a$ & computer disc & \\
\hline$b$ & $\mathrm{k}$ & $338 \neq a$ & computer card & \\
\hline $\mathrm{b}$ & $\mathrm{m}$ & $338 \neq a$ & computer disc & \\
\hline$b$ & 0 & $338 \neq a$ & computer disc & \\
\hline $\mathrm{b}$ & $r$ & $338 \neq a$ & online resource & \\
\hline$b$ & $\mathrm{u}$ & $338 \neq a$ & unspecified & \\
\hline$\underline{b}$ & $\mathrm{z}$ & $338 \neq a$ & other & \\
\hline $\bar{d}$ & $a$ & $300 \neq b$ & one color & Proposed RDA value; Concatenation required \\
\hline$d$ & $\mathrm{~b}$ & $300 \neq b$ & black and white & Concatenation required \\
\hline$d$ & c & $300 \neq b$ & color & Concatenation required \\
\hline$d$ & $\mathrm{~g}$ & $300 \neq b$ & gray scale & Proposed RDA value; Concatenation required \\
\hline$d$ & $\mathrm{~m}$ & $300 \neq b$ & mixed & Proposed RDA value; Concatenation required \\
\hline$d$ & $\mathrm{n}$ & & & No equivalent value \\
\hline$d$ & u & & & No equivalent value \\
\hline d & z & & & No equivalent value \\
\hline$\overline{\mathrm{e}}$ & $\mathrm{a}$ & $340 \neq b$ & $31 / 2$ in. & LC-PCC-PS (3.5.1.4.4) \\
\hline e & $\mathrm{e}$ & $340 \neq b$ & $12 \mathrm{in.}$ & LC-PCC-PS (3.5.1.4.4) \\
\hline e & g & $340 \neq b$ & $43 / 4$ in. & LC-PCC-PS (3.5.1.4.4) \\
\hline e & $\mathrm{i}$ & $340 \neq b$ & $11 / 8 \times 23 / 8 \mathrm{in}$ & Proposed RDA value \\
\hline e & j & $340 \neq b$ & $37 / 8 \times 2$ 1/2 in. & Proposed RDA value \\
\hline e & $\mathrm{n}$ & & & No equivalent value \\
\hline e & 0 & $340 \neq b$ & 5 1/4 in. & LC-PCC-PS (3.5.1.4.4) \\
\hline e & $\mathrm{u}$ & & & No equivalent value \\
\hline e & v & $340 \neq b$ & 8 in. & LC-PCC-PS (3.5.1.4.4) \\
\hline $\mathrm{e}$ & $\mathrm{z}$ & & & No equivalent value \\
\hline $\bar{f}$ & $\langle$ blank $>$ & & & \\
\hline$f$ & $\mathrm{a}$ & $300 \neq b$ & sound & Concatenation required \\
\hline$f$ & $\mathrm{u}$ & & & \\
\hline$\overline{\mathrm{g}}$ & \#\#\# & & & No equivalent element \\
\hline g & $\mathrm{mmm}$ & & & No equivalent element \\
\hline g & $\mathrm{nnn}$ & & & No equivalent element \\
\hline $\mathrm{g}$ & --- & & & No equivalent element \\
\hline $\bar{h}$ & $\mathrm{a}$ & & & No equivalent element \\
\hline $\mathrm{h}$ & $\mathrm{m}$ & & & No equivalent element \\
\hline $\mathrm{h}$ & $\mathrm{u}$ & & & No equivalent element \\
\hline$\overline{\mathrm{i}}$ & $a$ & & & No equivalent element \\
\hline i & $\mathrm{n}$ & & & No equivalent element \\
\hline i & $\mathrm{p}$ & & & No equivalent element \\
\hline $\mathrm{i}$ & $\mathrm{u}$ & & & No equivalent element \\
\hline $\bar{j}$ & $a$ & & & No equivalent element \\
\hline j & $\mathrm{b}$ & & & No equivalent element \\
\hline
\end{tabular}


MARC Reborn: Migrating MARC Fixed Field Metadata into the Variable Fields

Conversion Table for Electronic Resource (007 $¥ a=c)$

\begin{tabular}{lll} 
007 Subfield & Value & Variable Field Value \\
$\mathrm{j}$ & $\mathrm{c}$ & Notes \\
$\mathrm{j}$ & $\mathrm{d}$ & No equivalent element \\
$\mathrm{j}$ & $\mathrm{m}$ & No equivalent element \\
$\mathrm{j}$ & $\mathrm{n}$ & No equivalent element \\
$\mathrm{j}$ & $\mathrm{u}$ & No equivalent element \\
\hline $\mathrm{k}$ & $\mathrm{a}$ & No equivalent element \\
$\mathrm{k}$ & $\mathrm{b}$ & No equivalent element \\
$\mathrm{k}$ & $\mathrm{d}$ & No equivalent element \\
$\mathrm{k}$ & $\mathrm{m}$ & No equivalent element \\
$\mathrm{k}$ & $\mathrm{u}$ & No equivalent element \\
\hline $\mathrm{l}$ & $\mathrm{a}$ & No equivalent element \\
$\mathrm{l}$ & $\mathrm{n}$ & No equivalent element \\
$\mathrm{l}$ & $\mathrm{p}$ & No equivalent element \\
$\mathrm{l}$ & $\mathrm{r}$ & No equivalent element \\
$\mathrm{l}$ & $\mathrm{u}$ & No equivalent element
\end{tabular}


MARC Reborn: Migrating MARC Fixed Field Metadata into the Variable Fields

Conversion Table for Globe (007 $\ddagger \mathrm{a}=\mathrm{d}$ )

\begin{tabular}{|c|c|c|c|c|}
\hline 007 Subfield & Value & Variable Field & Value & Notes \\
\hline$b$ & $\mathrm{a}$ & $655 \ddagger a$ & Celestial globes & \\
\hline b & $\mathrm{b}$ & $655 \ddagger a$ & Planetary or lunar globes & Uncontrolled value... requires 2 nd indicator ' 4 ' \\
\hline b & c & $655 \neq a$ & Terrestrial globes & Uncontrolled value... requires 2 nd indicator ' 4 ' \\
\hline$b$ & $\mathrm{e}$ & $655 \neq a$ & Lunar globes & Uncontrolled value... requires 2 nd indicator ' 4 ' \\
\hline$b$ & $\mathrm{u}$ & & & No equivalent value \\
\hline $\mathrm{b}$ & z & & & No equivalent value \\
\hline $\bar{d}$ & a & $300 \mp b$ & one color & Proposed RDA value; Concatenation required \\
\hline d & c & $300 \neq b$ & color & Concatenation required \\
\hline$\overline{\mathrm{e}}$ & $\mathrm{a}$ & $340 \neq a$ & paper & \\
\hline e & $\mathrm{b}$ & $340 \neq a$ & wood & \\
\hline e & c & $340 \neq a$ & stone & \\
\hline e & $d$ & $340 \neq a$ & metal & \\
\hline e & $\mathrm{e}$ & $340 \neq a$ & synthetic & \\
\hline e & $f$ & $340 \neq a$ & skin & \\
\hline e & g & $340 \neq a$ & textile & \\
\hline e & $\mathrm{i}$ & $340 \neq a$ & plastic & \\
\hline e & I & $340 \neq a$ & vinyl & \\
\hline e & $\mathrm{n}$ & $340 \neq a$ & vellum & \\
\hline e & $\mathrm{p}$ & $340 \neq a$ & plaster & \\
\hline e & $\mathrm{u}$ & & & No equivalent value \\
\hline e & v & $340 \neq a$ & leather & \\
\hline e & w & $340 \neq a$ & parchment & \\
\hline e & $z$ & & & No equivalent value \\
\hline$f$ & $f$ & $340 \neq j$ & facsimile & Proposed RDA value \\
\hline$f$ & $\mathrm{n}$ & & & No equivalent value \\
\hline$f$ & $\mathrm{u}$ & & & No equivalent value \\
\hline$f$ & $z$ & & & No equivalent value \\
\hline
\end{tabular}


MARC Reborn: Migrating MARC Fixed Field Metadata into the Variable Fields

Conversion Table for Tactile Material (007 $\ddagger \mathrm{a}=\mathrm{f}$ )

\begin{tabular}{|c|c|c|c|c|}
\hline 007 Subfield & Value & Variable Field & Value & Notes \\
\hline$b$ & a & $546 \neq b$ & Moon code & \\
\hline$b$ & $\mathrm{~b}$ & $546 \neq b$ & braille code & \\
\hline b & c & & & No equivalent value \\
\hline$b$ & $d$ & $546 \neq b$ & tactile graphic & \\
\hline$b$ & $\mathrm{u}$ & & & No equivalent value \\
\hline$\underline{b}$ & z & & & No equivalent value \\
\hline $\bar{d}$ & $a$ & $546 \neq b$ & literary braille & Proposed RDA value \\
\hline$d$ & $\mathrm{~b}$ & $546 \neq b$ & format braille code & Proposed RDA value \\
\hline$d$ & c & $546 \neq b$ & mathematics braille code & \\
\hline$d$ & $d$ & $546 \neq b$ & computing braille code & \\
\hline$d$ & e & $546 \mp b$ & music braille code & \\
\hline$d$ & $\mathrm{~m}$ & & & No equivalent value \\
\hline$d$ & $\mathrm{n}$ & & & No equivalent value \\
\hline$d$ & $\mathrm{u}$ & & & No equivalent value \\
\hline d & $z$ & & & No equivalent value \\
\hline $\mathrm{e}$ & $a$ & & & No equivalent element \\
\hline e & $\mathrm{b}$ & & & No equivalent element \\
\hline e & $\mathrm{m}$ & & & No equivalent element \\
\hline e & $\mathrm{n}$ & & & No equivalent element \\
\hline e & $\mathrm{u}$ & & & No equivalent element \\
\hline $\mathrm{e}$ & $z$ & & & No equivalent element \\
\hline$f$ & $a$ & $340 \ddagger k$ & bar over bar & \\
\hline f & $\mathrm{b}$ & $340 \ddagger \mathrm{k}$ & bar by bar & \\
\hline f & c & $340 \ddagger k$ & line over line & \\
\hline f & $d$ & $340 \ddagger k$ & paragraph & \\
\hline$f$ & $\mathrm{e}$ & $340 \neq k$ & single line & \\
\hline f & $f$ & $340 \ddagger k$ & section by section & \\
\hline f & $\mathrm{g}$ & $340 \ddagger k$ & line by line & \\
\hline f & $\mathrm{h}$ & $340 \ddagger k$ & open score & \\
\hline f & $\mathrm{i}$ & $340 \ddagger k$ & melody chord system & \\
\hline f & j & $340 \ddagger k$ & short form scoring & \\
\hline$f$ & k & $340 \ddagger k$ & outline & \\
\hline f & I & $340 \ddagger \mathrm{k}$ & vertical score & \\
\hline$f$ & $\mathrm{n}$ & & & No equivalent value \\
\hline f & $\mathrm{u}$ & & & No equivalent value \\
\hline$f$ & $z$ & & & No equivalent value \\
\hline $\bar{g}$ & $a$ & $340 \ddagger n$ & Print/braille & Proposed RDA value \\
\hline g & $\mathrm{b}$ & $340 \neq n$ & jumbo braille & \\
\hline g & $\mathrm{n}$ & & & No equivalent value \\
\hline g & $\mathrm{u}$ & & & No equivalent value \\
\hline g & $z$ & & & No equivalent value \\
\hline
\end{tabular}


Conversion Table for Projected Graphic (007 $¥ \mathrm{a}=\mathrm{g}$ )

\begin{tabular}{|c|c|c|c|c|}
\hline 007 Subfield & Value & Variable Field & Value & Notes \\
\hline $\mathrm{b}$ & c & $338 \neq a$ & filmstrip cartridge & \\
\hline $\mathrm{b}$ & $d$ & $338 \neq a$ & filmslip & \\
\hline $\mathrm{b}$ & $f$ & $338 \neq a$ & filmstrip & \\
\hline $\mathrm{b}$ & 0 & $338 \neq a$ & film roll & \\
\hline $\mathrm{b}$ & s & $338 \neq a$ & slide & \\
\hline $\mathrm{b}$ & $\mathrm{t}$ & $338 \neq a$ & overhead transparency & \\
\hline $\mathrm{b}$ & $\mathrm{u}$ & $338 \neq a$ & unspecified & \\
\hline $\mathrm{b}$ & z & $338 \neq a$ & other & \\
\hline $\bar{d}$ & $\mathrm{a}$ & $300 \neq b$ & one color & Proposed RDA value; Concatenation required \\
\hline$d$ & $\mathrm{~b}$ & $300 \neq b$ & black and white & Concatenation required \\
\hline$d$ & c & $300 \neq b$ & color & Concatenation required \\
\hline$d$ & $\mathrm{~h}$ & $300 \mp b$ & hand-colored & Proposed RDA value; Concatenation required \\
\hline$d$ & $\mathrm{~m}$ & & & No equivalent value \\
\hline$d$ & $\mathrm{n}$ & & & No equivalent value \\
\hline$d$ & $\mathrm{u}$ & & & No equivalent value \\
\hline$d$ & z & & & No equivalent value \\
\hline$\overline{\mathrm{e}}$ & $\mathrm{d}$ & & & No equivalent element \\
\hline $\mathrm{e}$ & e & & & No equivalent element \\
\hline $\mathrm{e}$ & j & & & No equivalent element \\
\hline e & $\mathrm{k}$ & & & No equivalent element \\
\hline $\mathrm{e}$ & $\mathrm{m}$ & & & No equivalent element \\
\hline $\mathrm{e}$ & 0 & & & No equivalent element \\
\hline $\mathrm{e}$ & $\mathrm{u}$ & & & No equivalent element \\
\hline$\underline{\mathrm{e}}$ & $z$ & & & No equivalent element \\
\hline $\bar{f}$ & $\mathrm{a}$ & & & No equivalent element \\
\hline$f$ & $\mathrm{~b}$ & & & No equivalent element \\
\hline$f$ & $\mathrm{u}$ & & & No equivalent element \\
\hline$\overline{\mathrm{g}}$ & $a$ & & & No equivalent element \\
\hline g & $\mathrm{b}$ & & & No equivalent element \\
\hline $\mathrm{g}$ & c & & & No equivalent element \\
\hline $\mathrm{g}$ & $d$ & & & No equivalent element \\
\hline $\mathrm{g}$ & $\mathrm{e}$ & & & No equivalent element \\
\hline $\mathrm{g}$ & $f$ & & & No equivalent element \\
\hline $\mathrm{g}$ & g & & & No equivalent element \\
\hline $\mathrm{g}$ & $\mathrm{h}$ & & & No equivalent element \\
\hline g & $\mathrm{i}$ & & & No equivalent element \\
\hline g & $\mathrm{u}$ & & & No equivalent element \\
\hline$\underline{\mathrm{g}}$ & $z$ & & & No equivalent element \\
\hline $\bar{h}$ & $a$ & $340 \neq b$ & standard $8 \mathrm{~mm}$ & \\
\hline $\mathrm{h}$ & $\mathrm{b}$ & $340 \neq b$ & Super $8 \mathrm{~mm} /$ single $8 \mathrm{~mm}$ & Split Value \\
\hline $\mathrm{h}$ & c & $340 \ddagger b$ & $9.5 \mathrm{~mm}$ & \\
\hline $\mathrm{h}$ & $d$ & $340 \mp b$ & $16 \mathrm{~mm}$ & \\
\hline $\mathrm{h}$ & $\mathrm{e}$ & $340 \neq b$ & $28 \mathrm{~mm}$ & \\
\hline $\mathrm{h}$ & $f$ & $340 \neq b$ & $35 \mathrm{~mm}$ & \\
\hline $\mathrm{h}$ & $\mathrm{g}$ & $340 \ddagger b$ & $70 \mathrm{~mm}$ & \\
\hline $\mathrm{h}$ & j & $340 \neq b$ & $5 \times 5 \mathrm{~cm}$ & \\
\hline $\mathrm{h}$ & $\mathrm{k}$ & $340 \neq b$ & $6 \times 6 \mathrm{~cm}$ & \\
\hline $\mathrm{h}$ & $s$ & $340 \mp b$ & $10 \times 13 \mathrm{~cm}$ & \\
\hline
\end{tabular}


MARC Reborn: Migrating MARC Fixed Field Metadata into the Variable Fields

Conversion Table for Projected Graphic (007 $¥ \mathrm{a}=\mathrm{g})$

\begin{tabular}{lllll} 
007 Subfield & Value & Variable Field & Value & Notes \\
h & t & $340 \neq b$ & $13 \times 18 \mathrm{~cm}$ & \\
h & v & $340 \neq b$ & $21 \times 26 \mathrm{~cm}$ & \\
$\mathrm{~h}$ & $\mathrm{w}$ & $340 \neq \mathrm{b}$ & $23 \times 23 \mathrm{~cm}$ & \\
$\mathrm{~h}$ & $\mathrm{x}$ & $340 \neq \mathrm{b}$ & $26 \times 26 \mathrm{~cm}$ & No equivalent value \\
$\mathrm{h}$ & $\mathrm{y}$ & $340 \neq \mathrm{b}$ & $18 \times 18 \mathrm{~cm}$ & No equivalent value \\
$\mathrm{h}$ & $\mathrm{u}$ & & & \\
$\mathrm{h}$ & $\mathrm{z}$ & & & \\
\hline $\mathrm{i}$ & $\mathrm{c}$ & $340 \neq \mathrm{e}$ & cardboard & \\
$\mathrm{i}$ & $\mathrm{d}$ & $340 \neq \mathrm{e}$ & glass & \\
$\mathrm{i}$ & $\mathrm{e}$ & $340 \neq \mathrm{e}$ & synthetic & No equivalent value \\
$\mathrm{i}$ & $\mathrm{h}$ & $340 \neq \mathrm{e}$ & metal & No equivalent value \\
$\mathrm{i}$ & $\mathrm{j}$ & $340 \neq \mathrm{e}$ & metal, glass & No equivalent value \\
$\mathrm{i}$ & $\mathrm{k}$ & $340 \neq \mathrm{e}$ & synthetic, glass &
\end{tabular}


Conversion Table for Microform (007 $¥ a=h$ )

\begin{tabular}{|c|c|c|c|c|}
\hline 007 Subfield & Value & Variable Field & Value & Notes \\
\hline$b$ & $\mathrm{a}$ & 338 ‡a & aperture card & \\
\hline$b$ & $\mathrm{~b}$ & $338 \neq a$ & microfilm cartridge & \\
\hline$b$ & c & 338 ‡a & microfilm cassette & \\
\hline b & $d$ & $338 \neq a$ & microfilm reel & \\
\hline$b$ & $\mathrm{e}$ & 338 ‡a & microfiche & \\
\hline$b$ & $f$ & $338 \neq a$ & microfiche cassette & \\
\hline$b$ & $\mathrm{~g}$ & $338 \neq a$ & microopaque & \\
\hline$b$ & $\mathrm{~h}$ & $338 \neq a$ & microfilm slip & \\
\hline$b$ & j & $338 \neq a$ & microfilm roll & \\
\hline$b$ & $\mathrm{u}$ & $338 \neq a$ & unspecified & \\
\hline $\mathrm{b}$ & $\mathrm{z}$ & $338 \neq a$ & other & \\
\hline $\bar{d}$ & $\mathrm{a}$ & 340 ‡o & positive & \\
\hline$d$ & $\mathrm{~b}$ & 340 ‡o & negative & \\
\hline$d$ & $\mathrm{~m}$ & 340 ‡o & mixed polarity & \\
\hline d & $\mathrm{u}$ & & & No equivalent value \\
\hline$\overline{\mathrm{e}}$ & $a$ & $340 \neq b$ & $8 \mathrm{~mm}$ & \\
\hline e & $d$ & $340 \ddagger b$ & $16 \mathrm{~mm}$ & \\
\hline e & $f$ & $340 \neq b$ & $35 \mathrm{~mm}$ & \\
\hline e & $\mathrm{g}$ & $340 \ddagger b$ & $70 \mathrm{~mm}$ & \\
\hline e & $\mathrm{h}$ & $340 \ddagger b$ & $105 \mathrm{~mm}$ & \\
\hline e & I & $340 \mp b$ & $8 \times 13 \mathrm{~cm}$ & \\
\hline e & $\mathrm{m}$ & $340 \neq b$ & $11 \times 15 \mathrm{~cm}$ & \\
\hline e & 0 & $340 \ddagger b$ & $16 \times 23 \mathrm{~cm}$ & \\
\hline e & $\mathrm{p}$ & $340 \ddagger b$ & $9 \times 19 \mathrm{~cm}$ & \\
\hline e & $\mathrm{u}$ & & & No equivalent value \\
\hline $\mathrm{e}$ & $\mathrm{z}$ & & & No equivalent value \\
\hline$f$ & $a$ & $340 \neq f$ & low reduction & \\
\hline$f$ & $\mathrm{~b}$ & $340 \ddagger f$ & normal reduction & \\
\hline$f$ & c & $340 \neq f$ & high reduction & \\
\hline$f$ & $d$ & $340 \neq f$ & very high reduction & \\
\hline$f$ & $\mathrm{e}$ & $340 \neq f$ & ultra high reduction & \\
\hline$f$ & $\mathrm{u}$ & & & No equivalent value \\
\hline$f$ & $\mathrm{v}$ & $340 \neq f$ & reduction ratio varies & \\
\hline$\overline{\mathrm{g}}$ & $\mathrm{b}$ & $300 \neq b$ & black and white & Concatenation required \\
\hline g & c & $300 \ddagger b$ & color & Concatenation required \\
\hline g & $\mathrm{m}$ & & & No equivalent value \\
\hline g & $\mathrm{u}$ & & & No equivalent value \\
\hline g & $\mathrm{z}$ & & & No equivalent value \\
\hline $\mathrm{h}$ & $a$ & $340 \neq c$ & silver halide & \\
\hline $\mathrm{h}$ & $\mathrm{b}$ & $340 \neq c$ & diazo & \\
\hline $\mathrm{h}$ & c & $340 \neq c$ & vesicular & \\
\hline $\mathrm{h}$ & $\mathrm{m}$ & 340 ¥c & mixed & \\
\hline $\mathrm{h}$ & $\mathrm{n}$ & $340 \neq c$ & & No equivalent value \\
\hline $\mathrm{h}$ & $\mathrm{u}$ & $340 \neq c$ & & No equivalent value \\
\hline $\mathrm{h}$ & $\mathrm{z}$ & $340 \neq c$ & & No equivalent value \\
\hline $\bar{i}$ & $a$ & $340 \neq j$ & first generation & \\
\hline
\end{tabular}


MARC Reborn: Migrating MARC Fixed Field Metadata into the Variable Fields

Conversion Table for Microform (007 $¥ a=h$ )

\begin{tabular}{|c|c|c|c|c|}
\hline 007 Subfield & Value & Variable Field & Value & Notes \\
\hline $\mathrm{i}$ & $\mathrm{b}$ & $340 \neq j$ & printing master & \\
\hline $\mathrm{i}$ & c & 340 ‡j & service copy & \\
\hline $\mathrm{i}$ & $\mathrm{m}$ & $340 \neq j$ & mixed generation & \\
\hline$\underline{\mathrm{i}}$ & $\mathrm{u}$ & $340 \neq j$ & & No equivalent value \\
\hline $\bar{j}$ & $a$ & $340 \neq a$ & safety base & \\
\hline j & c & $340 \neq a$ & safety base, acetate & \\
\hline j & $d$ & $340 \neq a$ & safety base, diacetate & \\
\hline j & $\mathrm{i}$ & $340 \neq a$ & nitrate & \\
\hline j & $\mathrm{m}$ & $340 \neq a$ & nitrate, safety base & \\
\hline j & $\mathrm{n}$ & & & No equivalent value \\
\hline j & $\mathrm{p}$ & $340 \neq a$ & safety base, polyester & \\
\hline j & $r$ & $340 \neq a$ & safety base & \\
\hline j & $\mathrm{t}$ & $340 \neq a$ & safety base, triacetate & \\
\hline j & $\mathrm{u}$ & & & No equivalent value \\
\hline j & $z$ & & & No equivalent value \\
\hline
\end{tabular}


Conversion Table for Nonprojected Graphic (007 $¥ \mathrm{a}=\mathrm{k}$ )

\begin{tabular}{|c|c|c|c|c|}
\hline 007 Subfield & Value & Variable Field & Value & Notes \\
\hline$b$ & $a$ & $300 \neq f$ & activity card & \\
\hline$b$ & C & $300 \neq f$ & collage & \\
\hline$b$ & $d$ & $300 \neq f$ & drawing & \\
\hline$b$ & e & $300 \neq f$ & painting & \\
\hline$b$ & $f$ & & & No equivalent value \\
\hline$b$ & g & & & No equivalent value \\
\hline$b$ & $\mathrm{~h}$ & & & No equivalent value \\
\hline$b$ & $\mathrm{i}$ & $300 \neq f$ & picture & \\
\hline$b$ & j & $300 \ddagger f$ & print & \\
\hline$b$ & $\mathrm{k}$ & $300 \neq f$ & poster & \\
\hline$b$ & 1 & $300 \ddagger f$ & technical drawing & \\
\hline$b$ & $\mathrm{n}$ & $300 \neq f$ & chart & \\
\hline$b$ & 0 & $300 \neq f$ & flash card & \\
\hline$b$ & $\mathrm{p}$ & $300 \neq f$ & postcard & \\
\hline$b$ & $q$ & $300 \ddagger f$ & icon & \\
\hline$b$ & $r$ & $300 \neq f$ & radiograph & \\
\hline$b$ & s & $300 \ddagger f$ & study print & \\
\hline$b$ & $\mathrm{u}$ & & & No equivalent value \\
\hline$b$ & v & $300 \neq f$ & photograph & \\
\hline b & $z$ & & & No equivalent value \\
\hline $\bar{d}$ & $a$ & $300 \neq b$ & one color & Proposed RDA value \\
\hline$d$ & $b$ & $300 \neq b$ & black and white & \\
\hline$d$ & C & $300 \neq b$ & color & \\
\hline$d$ & $\mathrm{~h}$ & $300 \neq b$ & hand-colored & Proposed RDA value \\
\hline$d$ & $\mathrm{~m}$ & & & No equivalent value \\
\hline$d$ & $\mathrm{u}$ & & & No equivalent value \\
\hline$d$ & $z$ & & & No equivalent value \\
\hline$\overline{\mathrm{e}}$ & $a$ & $340 \neq a$ & canvas & \\
\hline e & $\mathrm{b}$ & $340 \neq a$ & bristol board & \\
\hline e & C & $340 \neq a$ & cardboard/illustration board & Split values \\
\hline e & $d$ & $340 \neq a$ & glass & \\
\hline e & $\mathrm{e}$ & $340 \neq a$ & synthetic & \\
\hline e & $f$ & $340 \neq a$ & skin & \\
\hline e & g & $340 \neq a$ & textile & \\
\hline e & $\mathrm{h}$ & $340 \neq a$ & metal & \\
\hline e & $\mathrm{i}$ & $340 \neq a$ & plastic & \\
\hline e & I & $340 \neq a$ & vinyl & \\
\hline e & $\mathrm{m}$ & & & No equivalent value \\
\hline e & $\mathrm{n}$ & $340 \neq a$ & vellum & \\
\hline e & 0 & $340 \neq a$ & paper & \\
\hline e & $\mathrm{p}$ & $340 \neq a$ & plaster & \\
\hline e & $q$ & $340 \neq a$ & hardboard & \\
\hline e & $r$ & $340 \neq a$ & porcelain & \\
\hline e & s & $340 \neq a$ & stone & \\
\hline e & $\mathrm{t}$ & $340 \neq a$ & wood & \\
\hline e & $\mathrm{u}$ & & & No equivalent value \\
\hline e & v & $340 \neq a$ & leather & \\
\hline e & w & $340 \neq a$ & parchment & \\
\hline
\end{tabular}


Conversion Table for Nonprojected Graphic (007 $¥ \mathrm{a}=\mathrm{k}$ )

\begin{tabular}{|c|c|c|c|c|}
\hline 007 Subfield & Value & Variable Field & Value & Notes \\
\hline e & $z$ & & & No equivalent value \\
\hline $\bar{f}$ & $\bar{a}$ & 340 ‡e & canvas & \\
\hline$f$ & $b$ & $340 \ddagger e$ & bristol board & \\
\hline$f$ & c & 340 ‡e & cardboard/illustration board & Split values \\
\hline$f$ & $d$ & $340 \ddagger e$ & glass & \\
\hline$f$ & e & 340 ‡e & synthetic & \\
\hline$f$ & $f$ & $340 \ddagger e$ & skin & \\
\hline$f$ & g & 340 ‡e & textile & \\
\hline$f$ & $\mathrm{~h}$ & $340 \ddagger e$ & metal & \\
\hline$f$ & $\mathrm{i}$ & 340 ‡e & plastic & \\
\hline$f$ & I & 340 ‡e & vinyl & \\
\hline$f$ & $\mathrm{~m}$ & & & No equivalent value \\
\hline$f$ & $\mathrm{n}$ & $340 \ddagger e$ & vellum & \\
\hline$f$ & o & $340 \ddagger e$ & paper & \\
\hline$f$ & $\mathrm{p}$ & $340 \ddagger e$ & plaster & \\
\hline$f$ & $q$ & 340 ‡e & hardboard & \\
\hline$f$ & $r$ & $340 \ddagger e$ & porcelain & \\
\hline$f$ & s & 340 ‡e & stone & \\
\hline$f$ & $\mathrm{t}$ & 340 ‡e & wood & \\
\hline$f$ & $\mathrm{u}$ & & & No equivalent value \\
\hline$f$ & $v$ & 340 ‡e & leather & \\
\hline$f$ & w & 340 ‡e & parchment & \\
\hline$f$ & $z$ & & & No equivalent value \\
\hline
\end{tabular}


Conversion Table for Motion Picture (007 $¥ \mathrm{a}=\mathrm{m})$

\begin{tabular}{|c|c|c|c|c|}
\hline 007 Subfield & Value & Variable Field & Value & Notes \\
\hline $\mathrm{b}$ & c & $338 \neq a$ & film cartridge & \\
\hline $\mathrm{b}$ & $f$ & $338 \neq a$ & film cassette & \\
\hline $\mathrm{b}$ & 0 & $338 \neq a$ & film roll & \\
\hline $\mathrm{b}$ & r & $338 \neq a$ & film reel & \\
\hline $\mathrm{b}$ & $\mathrm{u}$ & $338 \neq a$ & unspecified & \\
\hline $\mathrm{b}$ & z & $338 \neq a$ & other & \\
\hline $\bar{d}$ & $\mathrm{~b}$ & $300 \neq b$ & black and white & Concatenation required \\
\hline$d$ & c & $300 \neq b$ & color & Concatenation required \\
\hline$d$ & $\mathrm{~h}$ & $300 \mp b$ & hand-colored & Proposed RDA value; concatenation required \\
\hline$d$ & $\mathrm{~m}$ & & & No equivalent value \\
\hline$d$ & $\mathrm{n}$ & & & No equivalent value \\
\hline$d$ & u & & & No equivalent value \\
\hline $\mathrm{d}$ & z & & & No equivalent value \\
\hline$\overline{\mathrm{e}}$ & $a$ & $345 \neq a$ & standard sound aperture & \\
\hline $\mathrm{e}$ & $b$ & & & No equivalent value \\
\hline $\mathrm{e}$ & c & $345 \neq a$ & $3 D$ & \\
\hline $\mathrm{e}$ & $d$ & $345 \neq a$ & Techniscope/Panavision & Split values \\
\hline $\mathrm{e}$ & $\mathrm{e}$ & $345 \neq a$ & Cinerama/Cinemiracle/Cincarama & Split values \\
\hline e & $f$ & $345 \neq a$ & standard silent aperture & \\
\hline $\mathrm{e}$ & u & & & No equivalent value \\
\hline $\mathrm{e}$ & $z$ & & & No equivalent value \\
\hline $\bar{f}$ & $\mathrm{a}$ & & & No equivalent element \\
\hline$f$ & $b$ & & & No equivalent element \\
\hline$f$ & $\mathrm{u}$ & & & No equivalent element \\
\hline$\overline{\mathrm{g}}$ & 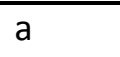 & & & No equivalent element \\
\hline g & $\mathrm{b}$ & & & No equivalent element \\
\hline g & c & & & No equivalent element \\
\hline g & $d$ & & & No equivalent element \\
\hline g & $\mathrm{e}$ & & & No equivalent element \\
\hline $\mathrm{g}$ & $f$ & & & No equivalent element \\
\hline $\mathrm{g}$ & $\mathrm{g}$ & & & No equivalent element \\
\hline g & $\mathrm{h}$ & & & No equivalent element \\
\hline g & $\mathrm{i}$ & & & No equivalent element \\
\hline g & $\mathrm{u}$ & & & No equivalent element \\
\hline$\underline{\mathrm{g}}$ & $z$ & & & No equivalent element \\
\hline $\bar{h}$ & $\mathrm{a}$ & $340 \neq b$ & standard $8 \mathrm{~mm}$ & \\
\hline $\mathrm{h}$ & $\mathrm{b}$ & $340 \neq b$ & Super $8 \mathrm{~mm} /$ single $8 \mathrm{~mm}$ & Split values \\
\hline $\mathrm{h}$ & c & $340 \ddagger b$ & $9.5 \mathrm{~mm}$ & \\
\hline $\mathrm{h}$ & $d$ & $340 \neq b$ & $16 \mathrm{~mm}$ & \\
\hline $\mathrm{h}$ & e & $340 \neq b$ & $28 \mathrm{~mm}$ & \\
\hline $\mathrm{h}$ & $f$ & $340 \neq b$ & $35 \mathrm{~mm}$ & \\
\hline $\mathrm{h}$ & g & $340 \neq b$ & $70 \mathrm{~mm}$ & \\
\hline $\mathrm{h}$ & $\mathrm{u}$ & & & No equivalent value \\
\hline $\mathrm{h}$ & $z$ & & & No equivalent value \\
\hline $\bar{i}$ & $\mathrm{k}$ & & & No equivalent element \\
\hline $\mathrm{i}$ & $\mathrm{m}$ & & & No equivalent element \\
\hline $\mathrm{i}$ & $q$ & & & No equivalent element \\
\hline $\mathrm{i}$ & s & & & No equivalent element \\
\hline
\end{tabular}


Conversion Table for Motion Picture (007 $¥ \mathrm{a}=\mathrm{m})$

\begin{tabular}{|c|c|c|c|c|}
\hline 007 Subfield & Value & Variable Field & Value & Notes \\
\hline $\mathrm{i}$ & $\mathrm{u}$ & & & No equivalent element \\
\hline$\underline{\mathrm{i}}$ & $z$ & & & No equivalent element \\
\hline $\bar{j}$ & $a$ & & & No equivalent element \\
\hline j & $b$ & & & No equivalent element \\
\hline j & c & & & No equivalent element \\
\hline j & $d$ & & & No equivalent element \\
\hline j & e & & & No equivalent element \\
\hline j & $f$ & & & No equivalent element \\
\hline j & g & & & No equivalent element \\
\hline j & $\mathrm{n}$ & & & No equivalent element \\
\hline j & z & & & No equivalent element \\
\hline $\bar{k}$ & $a$ & 340 ‡o & positive & \\
\hline$k$ & $b$ & 340 ‡o & negative & \\
\hline$k$ & $\mathrm{n}$ & & & No equivalent value \\
\hline $\mathrm{k}$ & u & & & No equivalent value \\
\hline $\mathrm{k}$ & z & & & No equivalent value \\
\hline $\bar{I}$ & $d$ & $340 \neq j$ & duplicate & \\
\hline I & e & $340 \neq j$ & master & \\
\hline I & o & $340 \neq j$ & original & \\
\hline I & $r$ & $340 \neq j$ & reference print/viewing copy & Split values \\
\hline I & u & & & No equivalent value \\
\hline$\underline{I}$ & z & & & No equivalent value \\
\hline$\overline{\mathrm{m}}$ & 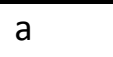 & $340 \neq a$ & safety base & \\
\hline $\mathrm{m}$ & c & $340 \neq a$ & safety base, acetate & \\
\hline $\mathrm{m}$ & $d$ & $340 \neq a$ & safety base, diacetate & \\
\hline $\mathrm{m}$ & $\mathrm{i}$ & $340 \neq a$ & nitrate & \\
\hline $\mathrm{m}$ & $\mathrm{m}$ & $340 \neq a$ & nitrate, safety base & \\
\hline $\mathrm{m}$ & $\mathrm{n}$ & & & No equivalent value \\
\hline $\mathrm{m}$ & $\mathrm{p}$ & 340 ‡a & safety base, polyester & \\
\hline $\mathrm{m}$ & r & $340 \neq a$ & safety base & \\
\hline $\mathrm{m}$ & $\mathrm{t}$ & $340 \neq a$ & safety base, triacetate & \\
\hline $\mathrm{m}$ & u & & & No equivalent value \\
\hline $\mathrm{m}$ & z & & & No equivalent value \\
\hline $\bar{n}$ & $a$ & & & No equivalent element \\
\hline $\mathrm{n}$ & $b$ & & & No equivalent element \\
\hline $\mathrm{n}$ & c & & & No equivalent element \\
\hline $\mathrm{n}$ & $d$ & & & No equivalent element \\
\hline $\mathrm{n}$ & e & & & No equivalent element \\
\hline $\mathrm{n}$ & $f$ & & & No equivalent element \\
\hline $\mathrm{n}$ & g & & & No equivalent element \\
\hline $\mathrm{n}$ & $\mathrm{h}$ & & & No equivalent element \\
\hline $\mathrm{n}$ & $\mathrm{i}$ & & & No equivalent element \\
\hline $\mathrm{n}$ & j & & & No equivalent element \\
\hline $\mathrm{n}$ & k & & & No equivalent element \\
\hline $\mathrm{n}$ & I & & & No equivalent element \\
\hline $\mathrm{n}$ & $\mathrm{m}$ & & & No equivalent element \\
\hline $\mathrm{n}$ & $\mathrm{n}$ & & & No equivalent element \\
\hline $\mathrm{n}$ & $\mathrm{p}$ & & & No equivalent element \\
\hline
\end{tabular}


Conversion Table for Motion Picture (007 $\neq \mathrm{a}=\mathrm{m})$

\begin{tabular}{|c|c|c|}
\hline 007 Subfield & Value Variable Field Value & Notes \\
\hline$n$ & $q$ & No equivalent element \\
\hline$n$ & $r$ & No equivalent element \\
\hline$n$ & s & No equivalent element \\
\hline$n$ & $\mathrm{t}$ & No equivalent element \\
\hline$n$ & $\mathrm{u}$ & No equivalent element \\
\hline$n$ & $\mathrm{v}$ & No equivalent element \\
\hline $\mathrm{n}$ & z & No equivalent element \\
\hline 0 & $a$ & No equivalent element \\
\hline o & $\mathrm{b}$ & No equivalent element \\
\hline 0 & c & No equivalent element \\
\hline 0 & $d$ & No equivalent element \\
\hline 0 & $\mathrm{n}$ & No equivalent element \\
\hline o & $\mathrm{u}$ & No equivalent element \\
\hline 0 & z & No equivalent element \\
\hline $\mathrm{p}$ & $\mathrm{a}$ & No equivalent element \\
\hline $\mathrm{p}$ & $\mathrm{b}$ & No equivalent element \\
\hline $\mathrm{p}$ & c & No equivalent element \\
\hline $\mathrm{p}$ & $d$ & No equivalent element \\
\hline $\mathrm{p}$ & e & No equivalent element \\
\hline $\mathrm{p}$ & $f$ & No equivalent element \\
\hline $\mathrm{p}$ & g & No equivalent element \\
\hline $\mathrm{p}$ & $\mathrm{h}$ & No equivalent element \\
\hline $\mathrm{p}$ & $\mathrm{k}$ & No equivalent element \\
\hline $\mathrm{p}$ & I & No equivalent element \\
\hline $\mathrm{p}$ & $\mathrm{m}$ & No equivalent element \\
\hline$q$ & C & No equivalent element \\
\hline$q$ & $\mathrm{i}$ & No equivalent element \\
\hline q & $\mathrm{n}$ & No equivalent element \\
\hline$q$ & $\mathrm{u}$ & No equivalent element \\
\hline$r$ & YYYYMM & No equivalent element \\
\hline
\end{tabular}


MARC Reborn: Migrating MARC Fixed Field Metadata into the Variable Fields

Conversion Table for Kit $(007 \ddagger a=0)$

007 Subfield Value Variable Field Value Notes

b

u $\quad 338 \neq a$

unspecified 
MARC Reborn: Migrating MARC Fixed Field Metadata into the Variable Fields

Conversion Table for Notated Music (007 $¥ \mathrm{a}=$ q)

007 Subfield Value Variable Field Value Notes

unspecified 
Conversion Table for Remote-sensing Image (007 $\neq a=r)$

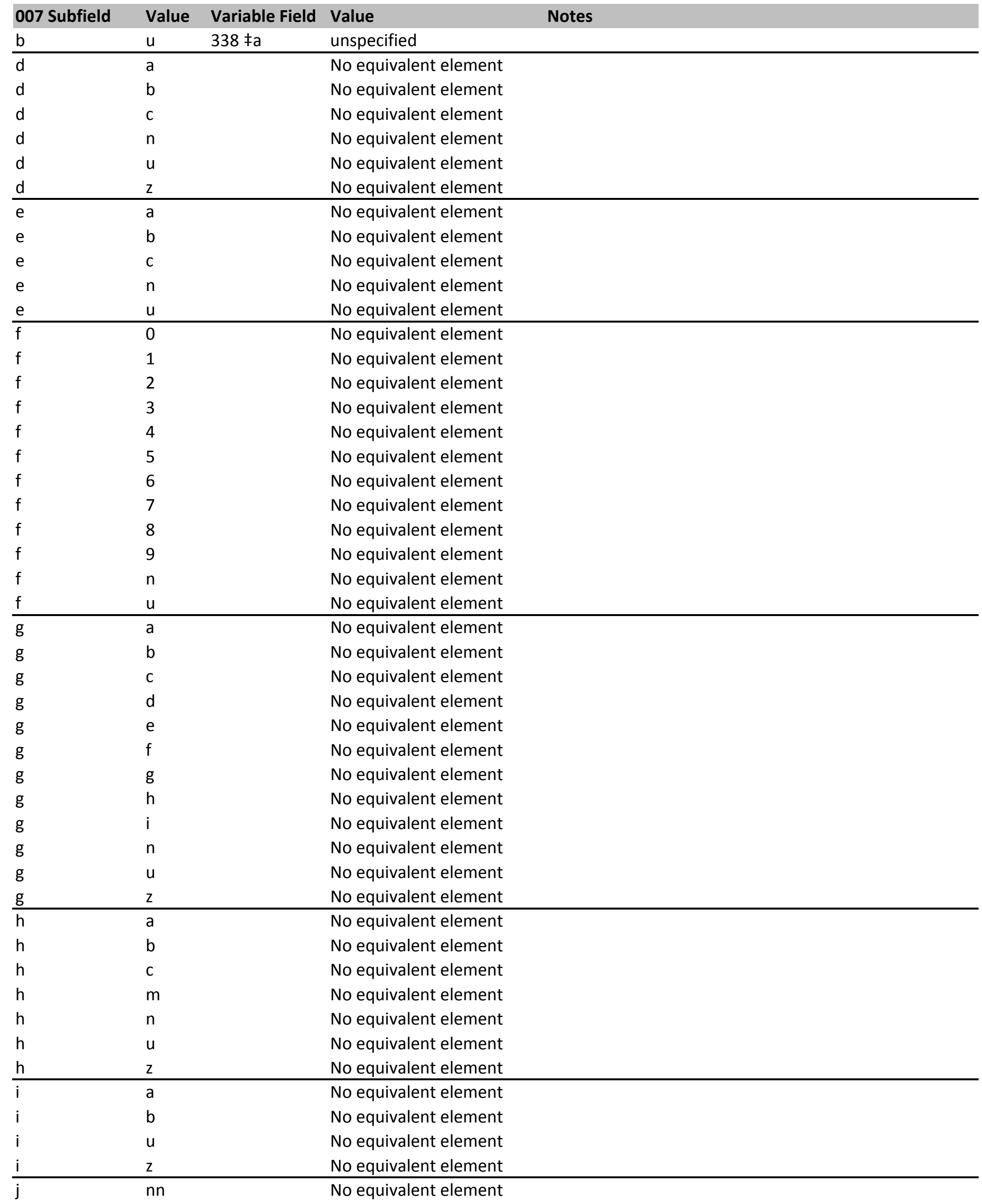


Conversion Table for Remote-sensing Image (007 $\neq a=r)$

\begin{tabular}{|c|c|c|c|}
\hline 007 Subfield & Value & Variable Field & Value \\
\hline j & uu & & No equivalent element \\
\hline j & $\mathrm{zz}$ & & No equivalent element \\
\hline j & aa & & No equivalent elemen \\
\hline j & da & & No equivalent elemer \\
\hline j & $\mathrm{db}$ & & No equivalent elemer \\
\hline j & dc & & No equivalent elemer \\
\hline j & dd & & No equivalent elemeı \\
\hline j & de & & No equivalent elemer \\
\hline j & $d f$ & & No equivalent elemer \\
\hline j & $\mathrm{dv}$ & & No equivalent eleme \\
\hline j & $\mathrm{dz}$ & & No equivalent eleme \\
\hline j & ga & & No equivalent eleme \\
\hline j & $\mathrm{gb}$ & & No equivalent eleme \\
\hline j & gc & & No equivalent eleme \\
\hline j & gd & & No equivalent eleme \\
\hline j & ge & & No equivalent elemer \\
\hline j & gf & & No equivalent elemer \\
\hline j & gg & & No equivalent eleme \\
\hline j & gu & & No equivalent eleme \\
\hline j & gz & & No equivalent eleme \\
\hline j & ja & & No equivalent eleme \\
\hline j & $\mathrm{jb}$ & & No equivalent eleme \\
\hline j & jc & & No equivalent eleme \\
\hline j & jv & & No equivalent elemer \\
\hline j & jz & & No equivalent elemeı \\
\hline j & ma & & No equivalent eleme \\
\hline j & $\mathrm{mb}$ & & No equivalent eleme \\
\hline j & $\mathrm{mm}$ & & No equivalent eleme \\
\hline j & pa & & No equivalent eleme \\
\hline j & $\mathrm{pb}$ & & No equivalent eleme \\
\hline j & $\mathrm{pc}$ & & No equivalent eleme \\
\hline j & $\mathrm{pd}$ & & No equivalent eleme \\
\hline j & pe & & No equivalent elemer \\
\hline j & $\mathrm{pz}$ & & No equivalent elemen \\
\hline j & ra & & No equivalent eleme \\
\hline j & $\mathrm{rb}$ & & No equivalent eleme \\
\hline j & rc & & No equivalent eleme \\
\hline j & rd & & No equivalent eleme \\
\hline j & sa & & No equivalent element \\
\hline j & ta & & No equivalent eleme \\
\hline
\end{tabular}


Conversion Table for Sound Recording (007 $¥ \mathrm{a}=\mathrm{s}$ )

\begin{tabular}{|c|c|c|c|c|}
\hline 007 Subfield & Value & Variable Field & Value & Notes \\
\hline$b$ & c & $338 \neq a$ & audio cylinder & \\
\hline$b$ & $d$ & $338 \neq a$ & audio disc & \\
\hline$b$ & e & $338 \neq a$ & audio cylinder & \\
\hline$b$ & $f$ & $338 \neq a$ & sound-track reel & \\
\hline$b$ & g & $338 \neq a$ & audio cartridge & \\
\hline$b$ & $\mathrm{i}$ & $338 \neq a$ & sound-track reel & \\
\hline$b$ & $q$ & $338 \neq a$ & audio roll & \\
\hline$b$ & $r$ & $338 \neq a$ & audiocassette & \\
\hline$b$ & s & $338 \neq a$ & audiocassette & \\
\hline$b$ & $\mathrm{t}$ & $338 \neq a$ & audiotape reel & \\
\hline$b$ & u & $338 \neq a$ & unspecified & \\
\hline$b$ & w & $338 \neq a$ & wire recording & Non-RDA value \\
\hline $\mathrm{b}$ & $z$ & $338 \neq a$ & other & \\
\hline $\bar{d}$ & $a$ & $344 \neq c$ & $16 \mathrm{rpm}$ & \\
\hline$d$ & $b$ & $344 \neq c$ & $331 / 3 \mathrm{rpm}$ & \\
\hline$d$ & c & $344 \neq c$ & $45 \mathrm{rpm}$ & \\
\hline$d$ & $d$ & $344 \neq c$ & $78 \mathrm{rpm}$ & \\
\hline$d$ & e & $344 \neq c$ & $8 \mathrm{rpm}$ & \\
\hline$d$ & $f$ & $344 \neq c$ & $1.4 \mathrm{~m} / \mathrm{s}$ & \\
\hline$d$ & $\mathrm{~h}$ & $344 \neq c$ & 120 rpm & \\
\hline$d$ & $\mathrm{i}$ & $344 \neq c$ & 160 rpm & \\
\hline$d$ & $k$ & $344 \neq c$ & $15 / 16$ ips & LC-PCC PS (3.16.4.3) \\
\hline$d$ & I & $344 \neq c$ & $17 / 8$ ips & LC-PCC PS (3.16.4.3) \\
\hline$d$ & $\mathrm{~m}$ & $344 \neq c$ & $33 / 4$ ips & LC-PCC PS (3.16.4.3) \\
\hline$d$ & 0 & $344 \neq c$ & 7 1/2 ips & LC-PCC PS (3.16.4.3) \\
\hline$d$ & $\mathrm{p}$ & $344 \neq c$ & $15 \mathrm{ips}$ & LC-PCC PS (3.16.4.3) \\
\hline$d$ & $r$ & $344 \neq c$ & 30 ips & LC-PCC PS (3.16.4.3) \\
\hline$d$ & $\mathrm{u}$ & & & No equivalent value \\
\hline d & $z$ & & & No equivalent value \\
\hline$\overline{\mathrm{e}}$ & $m$ & $344 \neq g$ & mono & \\
\hline e & $q$ & $344 \neq g$ & quadraphonic & \\
\hline e & s & $344 \neq g$ & stereo & \\
\hline e & $\mathrm{u}$ & & & No equivalent value \\
\hline $\mathrm{e}$ & $z$ & & & No equivalent value \\
\hline \multirow[t]{2}{*}{$f$} & $m$ & $344 \neq d$ & microgroove & If $007 \neq b$ d \\
\hline & & $344 \neq d$ & fine & If $007 \ddagger b$ c or e... \\
\hline$f$ & $\mathrm{n}$ & & & No equivalent value \\
\hline$f$ & s & $344 \neq d$ & coarse groove & If $007 \neq b d$ \\
\hline & & $344 \mp d$ & standard & If $007 \ddagger b$ c or $e . .$. \\
\hline$f$ & u & & & No equivalent value \\
\hline$f$ & z & & & No equivalent value \\
\hline$\overline{\mathrm{g}}$ & $a$ & $340 \neq b$ & 3 in. & LC-PCC PS (3.5.1.3); Concatenation required \\
\hline g & $\mathrm{b}$ & $340 \neq b$ & 5 in. & LC-PCC PS (3.5.1.3); Concatenation required \\
\hline g & c & $340 \neq b$ & $7 \mathrm{in.}$ & LC-PCC PS (3.5.1.3); Concatenation required \\
\hline g & $d$ & $340 \ddagger b$ & $10 \mathrm{in.}$ & LC-PCC PS (3.5.1.3); Concatenation required \\
\hline g & $\mathrm{e}$ & $340 \neq b$ & $12 \mathrm{in.}$ & LC-PCC PS (3.5.1.3); Concatenation required \\
\hline g & $f$ & $340 \ddagger b$ & $16 \mathrm{in.}$ & LC-PCC PS (3.5.1.3); Concatenation required \\
\hline g & g & $340 \neq b$ & $43 / 4$ in. $(12 \mathrm{~cm})$. & LC-PCC PS (3.5.1.3); Concatenation required \\
\hline
\end{tabular}




\section{Conversion Table for Sound Recording (007 $¥ \mathrm{a}=\mathrm{s}$ )}

\begin{tabular}{|c|c|c|c|c|}
\hline 007 Subfield & Value & Variable Field & Value & Notes \\
\hline g & j & $340 \neq b$ & $37 / 8 \times 21 / 2 \mathrm{in}$ & LC-PCC PS (3.5.1.3); Concatenation required \\
\hline $\mathrm{g}$ & 0 & $340 \mp b$ & $51 / 4 \times 37 / 8 \mathrm{in}$. & LC-PCC PS (3.5.1.3); Concatenation required \\
\hline $\mathrm{g}$ & s & $340 \mp b$ & $23 / 4 \times 4$ in. & LC-PCC PS (3.5.1.3); Concatenation required \\
\hline g & $\mathrm{n}$ & & & No equivalent value \\
\hline $\mathrm{g}$ & $\mathrm{u}$ & & & No equivalent value \\
\hline$\underline{\mathrm{g}}$ & z & & & No equivalent value \\
\hline $\mathrm{h}$ & $\mathrm{I}$ & $340 \mp b$ & $1 / 8$ in. & LC-PCC PS (3.5.1.3); Concatenation required \\
\hline $\mathrm{h}$ & $\mathrm{m}$ & $340 \neq b$ & $1 / 4$ in. & LC-PCC PS (3.5.1.3); Concatenation required \\
\hline $\mathrm{h}$ & $\mathrm{n}$ & & & No equivalent value \\
\hline $\mathrm{h}$ & 0 & $340 \neq b$ & $1 / 2$ in. & LC-PCC PS (3.5.1.3); Concatenation required \\
\hline $\mathrm{h}$ & $\mathrm{p}$ & $340 \mp b$ & $1 \mathrm{in.}$ & LC-PCC PS (3.5.1.3); Concatenation required \\
\hline $\mathrm{h}$ & $\mathrm{u}$ & & & No equivalent value \\
\hline$\underline{\mathrm{h}}$ & z & & & No equivalent value \\
\hline $\bar{i}$ & $\mathrm{a}$ & $344 \neq f$ & full (1) track & \\
\hline $\mathrm{i}$ & $\mathrm{b}$ & $344 \neq f$ & half (2) track & \\
\hline $\mathrm{i}$ & c & $344 \ddagger f$ & quarter (4) track & \\
\hline $\mathrm{i}$ & $d$ & $344 \ddagger f$ & 8 track & \\
\hline $\mathrm{i}$ & $\mathrm{e}$ & $344 \neq f$ & 12 track & \\
\hline $\mathrm{i}$ & $f$ & $344 \ddagger f$ & 16 track & \\
\hline $\mathrm{i}$ & $\mathrm{n}$ & & & No equivalent value \\
\hline $\mathrm{i}$ & $\mathrm{u}$ & & & No equivalent value \\
\hline$\underline{i}$ & $z$ & & & No equivalent value \\
\hline j & $\mathrm{a}$ & $340 \ddagger j$ & master tape & \\
\hline j & $\mathrm{b}$ & $340 \neq j$ & tape duplication master & \\
\hline j & $d$ & $340 \neq j$ & disc master & \\
\hline j & $\mathrm{i}$ & $340 \ddagger j$ & instantaneous & Non-RDA value \\
\hline j & $\mathrm{m}$ & $340 \ddagger j$ & mass produced & Non-RDA value \\
\hline $\mathrm{j}$ & $\mathrm{n}$ & & & No equivalent value \\
\hline j & $r$ & $340 \ddagger j$ & mother & \\
\hline j & s & $340 \neq j$ & stamper & \\
\hline j & $\mathrm{t}$ & $340 \neq j$ & test pressing & \\
\hline j & $\mathrm{u}$ & & & No equivalent value \\
\hline j & $z$ & & & No equivalent value \\
\hline$\overline{\mathrm{k}}$ & $\mathrm{a}$ & $340 \neq a$ & lacquer coating & Non-RDA value \\
\hline $\mathrm{k}$ & $\mathrm{b}$ & $340 \neq a$ & cellulose nitrate & Non-RDA value \\
\hline $\mathrm{k}$ & $\mathrm{C}$ & $340 \neq a$ & acetate tape with ferrous oxide & Non-RDA value \\
\hline k & g & $340 \neq a$ & glass with lacquer & Non-RDA value \\
\hline k & $\mathrm{i}$ & $340 \neq a$ & aluminum with lacquer & Non-RDA value \\
\hline k & 1 & $340 \neq a$ & metal & \\
\hline k & $\mathrm{m}$ & $340 \neq a$ & plastic with metal & Non-RDA value \\
\hline k & $\mathrm{p}$ & $340 \neq a$ & plastic & \\
\hline k & r & $340 \neq a$ & paper with lacquer or ferrous oxide & Non-RDA value \\
\hline k & s & $340 \ddagger a$ & shellac & \\
\hline k & $\mathrm{u}$ & & & No equivalent value \\
\hline k & w & $340 \neq a$ & wax & \\
\hline $\mathrm{k}$ & $\mathrm{z}$ & & & No equivalent value \\
\hline 1 & $\mathrm{~h}$ & & & No equivalent element \\
\hline 1 & I & & & No equivalent element \\
\hline
\end{tabular}


MARC Reborn: Migrating MARC Fixed Field Metadata into the Variable Fields

Conversion Table for Sound Recording (007 $¥ \mathrm{a}=\mathrm{s}$ )

\begin{tabular}{|c|c|c|c|c|}
\hline 007 Subfield & Value & Variable Field & Value & Notes \\
\hline I & $\mathrm{n}$ & & & No equivalent element \\
\hline I & $\mathrm{u}$ & & & No equivalent element \\
\hline$m$ & $\mathrm{a}$ & $344 \mp \mathrm{h}$ & NAB standard & \\
\hline $\mathrm{m}$ & $\mathrm{b}$ & $344 \neq h$ & CCIR standard & \\
\hline $\mathrm{m}$ & c & $344 \mp \mathrm{h}$ & Dolby-B encoded & \\
\hline $\mathrm{m}$ & $d$ & $344 \neq h$ & $\mathrm{dbx}$ encoded & \\
\hline $\mathrm{m}$ & $\mathrm{e}$ & $344 \ddagger h$ & digital recording & Proposed RDA value \\
\hline $\mathrm{m}$ & $f$ & $344 \neq h$ & Dolby-A encoded & \\
\hline $\mathrm{m}$ & g & $344 \ddagger h$ & Dolby-C encoded & \\
\hline $\mathrm{m}$ & $\mathrm{h}$ & $344 \ddagger h$ & CX encoded & \\
\hline $\mathrm{m}$ & $\mathrm{n}$ & & & No equivalent value \\
\hline $\mathrm{m}$ & $\mathrm{u}$ & & & No equivalent value \\
\hline $\mathrm{m}$ & $z$ & & & No equivalent value \\
\hline$n$ & $a$ & & & No equivalent element \\
\hline$n$ & $\mathrm{~b}$ & & & No equivalent element \\
\hline$n$ & $d$ & & & No equivalent element \\
\hline$n$ & $\mathrm{e}$ & & & No equivalent element \\
\hline $\mathrm{n}$ & $\mathrm{u}$ & & & No equivalent element \\
\hline$n$ & z & & & No equivalent element \\
\hline
\end{tabular}


MARC Reborn: Migrating MARC Fixed Field Metadata into the Variable Fields

Conversion Table for Text $(007 \ddagger \mathrm{a}=\mathrm{t})$

$\begin{array}{lllll}\text { 007 Subfield } & \text { Value } & \text { Variable Field } & \text { Value } & \text { Notes } \\ \text { b } & \text { a } & 340 \neq n & & \text { No equivalent value } \\ \text { b } & \text { b } & 340 \neq n & \text { large print } & \\ \text { b } & \text { c } & 546 \neq b & \text { braille code } & \\ \text { b } & \text { volume (loose-leaf) } & \\ \text { b } & \text { u } & 300 \neq f & & \text { No equivalent value } \\ \text { b } & \text { z } & & & \text { No equivalent value }\end{array}$


Conversion Table for Videorecording (007 $¥ \mathrm{a}=\mathrm{v}$ )

\begin{tabular}{|c|c|c|c|c|}
\hline 007 Subfield & Value & Variable Field & Value & Notes \\
\hline $\mathrm{b}$ & c & $338 \neq a$ & video cartridge & \\
\hline $\mathrm{b}$ & $d$ & $338 \neq a$ & videodisc & \\
\hline $\mathrm{b}$ & $f$ & $338 \neq a$ & videocassette & \\
\hline $\mathrm{b}$ & $\mathrm{n}$ & $338 \neq a$ & other & \\
\hline $\mathrm{b}$ & r & $338 \neq a$ & videotape reel & \\
\hline $\mathrm{b}$ & u & $338 \neq a$ & unspecified & \\
\hline $\mathrm{b}$ & z & $338 \neq a$ & other & \\
\hline $\bar{d}$ & $\mathrm{a}$ & & & No equivalent value \\
\hline$d$ & $b$ & $300 \neq b$ & black and white & Concatenation required \\
\hline$d$ & c & $300 \neq b$ & color & Concatenation required \\
\hline$d$ & $\mathrm{~m}$ & $300 \neq b$ & color with black and white sequences & Concatenation required \\
\hline$d$ & $\mathrm{n}$ & & & No equivalent value \\
\hline$d$ & $\mathrm{u}$ & & & No equivalent value \\
\hline$d$ & $z$ & & & No equivalent value \\
\hline$\overline{\mathrm{e}}$ & $a$ & $346 \neq a$ & Beta & \\
\hline $\mathrm{e}$ & $b$ & $346 \neq a$ & VHS & \\
\hline $\mathrm{e}$ & c & $346 \neq a$ & U-matic & \\
\hline e & $d$ & $346 \neq a$ & EIAJ & \\
\hline $\mathrm{e}$ & e & $346 \neq a$ & Type C & \\
\hline e & $f$ & $346 \neq a$ & Quadruplex & \\
\hline $\mathrm{e}$ & g & $346 \neq a$ & Laser optical & \\
\hline $\mathrm{e}$ & $\mathrm{h}$ & $346 \neq a$ & CED & \\
\hline $\mathrm{e}$ & $\mathrm{i}$ & $346 \neq a$ & Betacam & \\
\hline e & j & $346 \neq a$ & Betacam SP & \\
\hline $\mathrm{e}$ & $\mathrm{k}$ & $346 \neq a$ & Super-VHS & \\
\hline $\mathrm{e}$ & $\mathrm{m}$ & $346 \neq a$ & $M-I I$ & \\
\hline $\mathrm{e}$ & $\mathrm{n}$ & & & No equivalent value \\
\hline $\mathrm{e}$ & 0 & $346 \neq a$ & $D-2$ & \\
\hline $\mathrm{e}$ & $\mathrm{p}$ & $346 \neq a$ & $8 \mathrm{~mm}$ & \\
\hline $\mathrm{e}$ & $q$ & $346 \neq a$ & $\mathrm{Hi}-8 \mathrm{~mm}$ & \\
\hline $\mathrm{e}$ & s & $347 \neq b$ & Blu-ray & \\
\hline e & u & & & No equivalent value \\
\hline $\mathrm{e}$ & $v$ & $347 \neq b$ & DVD video & \\
\hline $\mathrm{e}$ & $z$ & & & No equivalent value \\
\hline $\bar{f}$ & $a$ & & & No equivalent element \\
\hline$f$ & $b$ & & & No equivalent element \\
\hline$f$ & $\mathrm{u}$ & & & No equivalent element \\
\hline$\overline{\mathrm{g}}$ & $a$ & & & No equivalent element \\
\hline g & $b$ & & & No equivalent element \\
\hline g & c & & & No equivalent element \\
\hline $\mathrm{g}$ & $d$ & & & No equivalent element \\
\hline g & e & & & No equivalent element \\
\hline g & $f$ & & & No equivalent element \\
\hline $\mathrm{g}$ & g & & & No equivalent element \\
\hline $\mathrm{g}$ & $\mathrm{h}$ & & & No equivalent element \\
\hline $\mathrm{g}$ & $\mathrm{i}$ & & & No equivalent element \\
\hline g & u & & & No equivalent element \\
\hline $\mathrm{g}$ & $z$ & & & No equivalent element \\
\hline
\end{tabular}


Conversion Table for Videorecording (007 $¥ \mathrm{a}=\mathrm{v}$ )

\begin{tabular}{lllll} 
007 Subfield & Value & Variable Field & Value & Notes \\
h & $\mathrm{a}$ & $340 \neq \mathrm{b}$ & $8 \mathrm{~mm}$ & \\
$\mathrm{~h}$ & $\mathrm{~m}$ & $340 \neq \mathrm{b}$ & $7 \mathrm{~mm}$ & \\
$\mathrm{~h}$ & $\mathrm{o}$ & $340 \neq \mathrm{b}$ & $13 \mathrm{~mm}$ & \\
$\mathrm{~h}$ & $\mathrm{p}$ & $340 \neq \mathrm{b}$ & $26 \mathrm{~mm}$ & \\
$\mathrm{~h}$ & $\mathrm{q}$ & $340 \neq \mathrm{b}$ & $51 \mathrm{~mm}$ & \\
$\mathrm{~h}$ & $\mathrm{r}$ & $340 \neq \mathrm{b}$ & $20 \mathrm{~mm}$ & \\
$\mathrm{~h}$ & $\mathrm{u}$ & $340 \neq \mathrm{b}$ & & No equivalent element \\
$\mathrm{h}$ & $\mathrm{z}$ & $340 \neq \mathrm{b}$ & & No equivalent element \\
\hline $\mathrm{i}$ & $\mathrm{k}$ & & & No equivalent element \\
$\mathrm{i}$ & $\mathrm{m}$ & & & No equivalent element \\
$\mathrm{i}$ & $\mathrm{n}$ & & & No equivalent element \\
$\mathrm{i}$ & $\mathrm{q}$ & & & No equivalent element \\
$\mathrm{i}$ & $\mathrm{s}$ & & & No equivalent element \\
$\mathrm{i}$ & $\mathrm{u}$ & & &
\end{tabular}


MARC Reborn: Migrating MARC Fixed Field Metadata into the Variable Fields

Conversion Table for Unspecified (007 $\neq \mathrm{a}=\mathrm{z}$ )

$\begin{array}{llll}\text { 007 Subfield } & \text { Value } & \text { Variable Field Value } & \text { Notes } \\ \text { b } & \mathrm{m} & & \text { No equivalent element } \\ \mathrm{b} & \mathrm{u} & & \text { No equivalent element } \\ \mathrm{b} & \mathrm{z} & & \text { No equivalent element }\end{array}$


Conversion Table for the Fixed Field

\begin{tabular}{|c|c|c|c|c|}
\hline \multicolumn{2}{|c|}{ Fixed Field Value } & \multicolumn{2}{|c|}{ Variable Field Value } & \multirow{2}{*}{$\begin{array}{l}\text { Notes } \\
\text { No equivalent element }\end{array}$} \\
\hline AccM & $<$ blank> & & & \\
\hline AccM & $a$ & & & No equivalent element \\
\hline AccM & $\mathrm{b}$ & & & No equivalent element \\
\hline AccM & c & & & No equivalent element \\
\hline AccM & $d$ & & & No equivalent element \\
\hline AccM & $\mathrm{e}$ & & & No equivalent element \\
\hline AccM & $f$ & & & No equivalent element \\
\hline AccM & $\mathrm{g}$ & & & No equivalent element \\
\hline AccM & $\mathrm{h}$ & & & No equivalent element \\
\hline AccM & $\mathrm{i}$ & & & No equivalent element \\
\hline AccM & $\mathrm{k}$ & & & No equivalent element \\
\hline AccM & $r$ & & & No equivalent element \\
\hline AccM & $\mathrm{s}$ & & & No equivalent element \\
\hline AccM & $\mathrm{z}$ & & & No equivalent element \\
\hline Alph & \multicolumn{3}{|l|}{$<$ blank $>$} & No equivalent value \\
\hline Alph & $a$ & $546 \neq b$ & Latin & LC-PCC PS (7.13.2.3) \\
\hline Alph & $b$ & $546 \neq b$ & Latin & Merged value; LC-PCC PS (7.13.2.3) \\
\hline Alph & c & $546 \neq b$ & Cyrillic & LC-PCC PS (7.13.2.3) \\
\hline Alph & $d$ & $546 \neq b$ & Japanese & LC-PCC PS (7.13.2.3) \\
\hline Alph & e & $546 \neq b$ & Han & LC-PCC PS (7.13.2.3) \\
\hline Alph & $f$ & $546 \neq b$ & Arabic & LC-PCC PS (7.13.2.3) \\
\hline Alph & $\mathrm{g}$ & $546 \neq b$ & Greek & LC-PCC PS (7.13.2.3) \\
\hline Alph & $\mathrm{h}$ & $546 \neq b$ & Hebrew & LC-PCC PS (7.13.2.3) \\
\hline Alph & $\mathrm{i}$ & $546 \neq b$ & Thai & LC-PCC PS (7.13.2.3) \\
\hline Alph & j & $546 \neq b$ & Devanagari & LC-PCC PS (7.13.2.3) \\
\hline Alph & $\mathrm{k}$ & $546 \neq b$ & Korean & LC-PCC PS (7.13.2.3) \\
\hline Alph & 1 & $546 \neq b$ & Tamil & LC-PCC PS (7.13.2.3) \\
\hline Alph & u & & & No equivalent value \\
\hline Alph & $\mathrm{z}$ & & & No equivalent value \\
\hline Audn & \multicolumn{3}{|l|}{$<$ blank> } & No equivalent value \\
\hline Audn & $a$ & $385 \neq a$ & preschool & Proposed RDA value \\
\hline Audn & $\mathrm{b}$ & $385 \neq a$ & primary & Proposed RDA value \\
\hline Audn & c & $385 \neq a$ & pre-adolescent & Proposed RDA value \\
\hline Audn & $d$ & $385 \neq a$ & adolescent & Proposed RDA value \\
\hline Audn & $\mathrm{e}$ & $385 \neq a$ & adult & Proposed RDA value \\
\hline Audn & $f$ & $385 \neq a$ & specialized & Proposed RDA value \\
\hline Audn & $\mathrm{g}$ & $385 \neq a$ & general & Proposed RDA value \\
\hline Audn & j & $385 \neq a$ & juvenile & Proposed RDA value \\
\hline Biog & \multicolumn{3}{|l|}{$<$ blank $>$} & No equivalent element \\
\hline Biog & \multicolumn{3}{|l|}{$\mathrm{a}$} & No equivalent element \\
\hline Biog & \multicolumn{3}{|l|}{$b$} & No equivalent element \\
\hline Biog & \multicolumn{3}{|l|}{ c } & No equivalent element \\
\hline Biog & \multicolumn{3}{|l|}{ d } & No equivalent element \\
\hline$\overline{\mathrm{BLvl}}$ & \multicolumn{3}{|l|}{$a$} & No equivalent value; No equivalent Field \\
\hline BLvl & \multicolumn{3}{|l|}{$\mathrm{b}$} & No equivalent value; No equivalent Field \\
\hline BLvl & \multicolumn{3}{|l|}{ c } & No equivalent value; No equivalent Field \\
\hline BLvl & \multicolumn{3}{|l|}{$d$} & No equivalent value; No equivalent Field \\
\hline BLvl & $\mathrm{i}$ & & integrating resource & No equivalent Field \\
\hline
\end{tabular}


Conversion Table for the Fixed Field

\begin{tabular}{|c|c|c|c|}
\hline \multicolumn{2}{|c|}{ Fixed Field Value } & \multirow{2}{*}{$\begin{array}{l}\text { Variable Field Value } \\
\text { single unit }\end{array}$} & \multirow{2}{*}{$\begin{array}{l}\text { Notes } \\
\text { No equivalent Field }\end{array}$} \\
\hline BLvl & $\mathrm{m}$ & & \\
\hline BLvl & $\mathrm{s}$ & serial & No equivalent Field \\
\hline Comp & an & & No equivalent element \\
\hline Comp & $\mathrm{bd}$ & & No equivalent element \\
\hline Comp & bt & & No equivalent element \\
\hline Comp & bg & & No equivalent element \\
\hline Comp & bl & & No equivalent element \\
\hline Comp & $\mathrm{cn}$ & & No equivalent element \\
\hline Comp & $\mathrm{ct}$ & & No equivalent element \\
\hline Comp & $\mathrm{Cz}$ & & No equivalent element \\
\hline Comp & $\mathrm{cr}$ & & No equivalent element \\
\hline Comp & $\mathrm{ca}$ & & No equivalent element \\
\hline Comp & CS & & No equivalent element \\
\hline Comp & $\mathrm{cp}$ & & No equivalent element \\
\hline Comp & cc & & No equivalent element \\
\hline Comp & $\mathrm{cb}$ & & No equivalent element \\
\hline Comp & $\mathrm{cl}$ & & No equivalent element \\
\hline Comp & ch & & No equivalent element \\
\hline Comp & $\mathrm{cg}$ & & No equivalent element \\
\hline Comp & co & & No equivalent element \\
\hline Comp & cy & & No equivalent element \\
\hline Comp & $\mathrm{df}$ & & No equivalent element \\
\hline Comp & $d v$ & & No equivalent element \\
\hline Comp & $\mathrm{ft}$ & & No equivalent element \\
\hline Comp & $\mathrm{fl}$ & & No equivalent element \\
\hline Comp & $\mathrm{fm}$ & & No equivalent element \\
\hline Comp & $\mathrm{fg}$ & & No equivalent element \\
\hline Comp & $\mathrm{gm}$ & & No equivalent element \\
\hline Comp & hy & & No equivalent element \\
\hline Comp & jz & & No equivalent element \\
\hline Comp & $\mathrm{md}$ & & No equivalent element \\
\hline Comp & $\mathrm{mr}$ & & No equivalent element \\
\hline Comp & $\mathrm{ms}$ & & No equivalent element \\
\hline Comp & $\mathrm{mz}$ & & No equivalent element \\
\hline Comp & $\mathrm{mi}$ & & No equivalent element \\
\hline Comp & mo & & No equivalent element \\
\hline Comp & $\mathrm{mp}$ & & No equivalent element \\
\hline Comp & $\mathrm{mu}$ & & No equivalent element \\
\hline Comp & $\mathrm{mc}$ & & No equivalent element \\
\hline Comp & nc & & No equivalent element \\
\hline Comp & $\mathrm{nn}$ & & No equivalent element \\
\hline Comp & op & & No equivalent element \\
\hline Comp & or & & No equivalent element \\
\hline Comp & ov & & No equivalent element \\
\hline Comp & $\mathrm{pt}$ & & No equivalent element \\
\hline Comp & ps & & No equivalent element \\
\hline Comp & $\mathrm{pm}$ & & No equivalent element \\
\hline Comp & $\mathrm{pv}$ & & No equivalent element \\
\hline
\end{tabular}


Conversion Table for the Fixed Field

\begin{tabular}{|c|c|c|c|c|}
\hline \multicolumn{2}{|c|}{ Fixed Field Value } & \multicolumn{2}{|c|}{ Variable Field Value } & \multirow{2}{*}{$\begin{array}{l}\text { Notes } \\
\text { No equivalent element }\end{array}$} \\
\hline Comp & po & & & \\
\hline Comp & $\mathrm{pp}$ & & & No equivalent element \\
\hline Comp & $\mathrm{pr}$ & & & No equivalent element \\
\hline Comp & pg & & & No equivalent element \\
\hline Comp & $\mathrm{rg}$ & & & No equivalent element \\
\hline Comp & $\mathrm{rq}$ & & & No equivalent element \\
\hline Comp & $\mathrm{rp}$ & & & No equivalent element \\
\hline Comp & ri & & & No equivalent element \\
\hline Comp & rc & & & No equivalent element \\
\hline Comp & rd & & & No equivalent element \\
\hline Comp & sn & & & No equivalent element \\
\hline Comp & sg & & & No equivalent element \\
\hline Comp & sd & & & No equivalent element \\
\hline Comp & st & & & No equivalent element \\
\hline Comp & su & & & No equivalent element \\
\hline Comp & $\mathrm{sp}$ & & & No equivalent element \\
\hline Comp & sy & & & No equivalent element \\
\hline Comp & $\mathrm{tl}$ & & & No equivalent element \\
\hline Comp & tc & & & No equivalent element \\
\hline Comp & ts & & & No equivalent element \\
\hline Comp & uu & & & No equivalent element \\
\hline Comp & vi & & & No equivalent element \\
\hline Comp & $\mathrm{vr}$ & & & No equivalent element \\
\hline Comp & wz & & & No equivalent element \\
\hline Comp & za & & & No equivalent element \\
\hline Comp & $\mathrm{zz}$ & & & No equivalent element \\
\hline Conf & $\cdot$ & & & No equivalent value \\
\hline Conf & 0 & & & No equivalent value \\
\hline Conf & 1 & $655 \neq a$ & Conference papers and proceedings & Genre Term (LCGFT) \\
\hline Cont & <blank> & & & \\
\hline Cont & $\mathrm{a}$ & $655 \neq a$ & Abstracts & Genre Term (LCGFT) \\
\hline Cont & $\mathrm{b}$ & $655 \neq a$ & Bibliographies & Genre Term (LCGFT) \\
\hline Cont & c & $655 \neq a$ & Catalogs & Genre Term (LCGFT) \\
\hline Cont & $d$ & $655 \neq a$ & Dictionaries & Genre Term (LCGFT) \\
\hline Cont & $\mathrm{e}$ & $655 \neq a$ & Encyclopedias & Genre Term (LCGFT) \\
\hline Cont & $f$ & $655 \neq a$ & Handbooks and manuals & Genre Term (LCGFT) \\
\hline Cont & g & $655 \neq a$ & Law reviews & Genre Term (LCGFT) \\
\hline Cont & $\mathrm{h}$ & $655 \neq a$ & Biographies & Genre Term (LCGFT) \\
\hline Cont & $\mathrm{i}$ & $655 \neq a$ & Indexes & Genre Term (LCGFT) \\
\hline Cont & j & $655 \neq a$ & Patents & Genre Term (LCGFT) \\
\hline Cont & $\mathrm{k}$ & $655 \neq a$ & Discographies & Genre Term (LCGFT) \\
\hline Cont & I & $655 \neq a$ & Statutes and codes & Genre Term (LCGFT) \\
\hline Cont & $\mathrm{m}$ & $655 \neq a$ & Academic theses & Genre Term (LCGFT) \\
\hline Cont & $\mathrm{n}$ & & & No equivalent value \\
\hline Cont & 0 & $655 \neq a$ & Reviews & Genre Term (LCGFT) \\
\hline Cont & $\mathrm{p}$ & $655 \neq a$ & Programmed instructional materials & Genre Term (LCGFT) \\
\hline Cont & $q$ & & & No equivalent value \\
\hline Cont & $r$ & $655 \neq a$ & Directories & Genre Term (LCGFT) \\
\hline
\end{tabular}


Conversion Table for the Fixed Field

\begin{tabular}{|c|c|c|c|c|}
\hline \multicolumn{2}{|c|}{ Fixed Field Value } & \multicolumn{2}{|c|}{ Variable Field Value } & \multirow{2}{*}{$\begin{array}{l}\text { Notes } \\
\text { Genre Term (LCGFT) }\end{array}$} \\
\hline Cont & $\mathrm{s}$ & $655 \neq a$ & Statistics & \\
\hline Cont & $\mathrm{t}$ & & & No equivalent value \\
\hline Cont & u & & & No equivalent value \\
\hline Cont & $\mathrm{v}$ & $655 \neq a$ & Casebooks & Genre Term (LCGFT) \\
\hline Cont & w & $655 \neq a$ & Court decisions and opinions & Genre Term (LCGFT) \\
\hline Cont & $x$ & & & No equivalent value \\
\hline Cont & $\mathrm{y}$ & $655 \neq a$ & Yearbooks & Genre Term (LCGFT) \\
\hline Cont & z & $655 \neq a$ & Treaties & Genre Term (LCGFT) \\
\hline Cont & 2 & & & No equivalent value \\
\hline Cont & 5 & $655 \neq a$ & Calendars & Genre Term (LCGFT) \\
\hline Cont & 6 & $655 \neq a$ & Comics (Graphic works) & Genre Term (LCGFT) \\
\hline$\overline{C r T p}$ & $a$ & $300 \neq f$ & map & \\
\hline CrTp & $b$ & & & No equivalent value \\
\hline CrTp & c & & & No equivalent value \\
\hline CrTp & $d$ & $300 \neq f$ & globe & \\
\hline CrTp & $\mathrm{e}$ & $300 \neq f$ & atlas & \\
\hline CrTp & $f$ & & & No equivalent value \\
\hline CrTp & $\mathrm{g}$ & & & No equivalent value \\
\hline CrTp & u & & & No equivalent value \\
\hline CrTp & $\mathrm{z}$ & & & No equivalent value \\
\hline$\overline{C t r l}$ & <blank> & & & Administrative Metadata \\
\hline Ctrl & $\mathrm{a}$ & & & Administrative Metadata \\
\hline Ctry & $\mathrm{aa}$ & $751 \neq a$ & Albania & Name Term (NAF) \\
\hline Ctry & $a b c$ & 751 ‡a & Alberta & Name Term (NAF) \\
\hline Ctry & $-a c$ & $751 \neq a$ & Ashmore and Cartier Islands & Name Term (NAF) \\
\hline Ctry & aca & 751 ‡a & Australian Capital Territory & Name Term (NAF) \\
\hline Ctry & ae & 751 ‡a & Algeria & Name Term (NAF) \\
\hline Ctry & af & 751 ‡a & Afghanistan & Name Term (NAF) \\
\hline Ctry & ag & 751 ‡a & Argentina & Name Term (NAF) \\
\hline Ctry & -ai & 751 ‡a & Anguilla & Name Term (NAF) \\
\hline Ctry & ai & 751 ‡a & Armenia (Republic) & Name Term (NAF) \\
\hline Ctry & -air & 751 ‡a & Armenian S.S.R. & Name Term (NAF) \\
\hline Ctry & aj & 751 ‡a & Azerbaijan & Name Term (NAF) \\
\hline Ctry & -ajr & 751 ‡a & Azerbaijan S.S.R. & Name Term (NAF) \\
\hline Ctry & aku & 751 ‡a & Alaska & Name Term (NAF) \\
\hline Ctry & alu & $751 \neq a$ & Alabama & Name Term (NAF) \\
\hline Ctry & am & 751 ‡a & Anguilla & Name Term (NAF) \\
\hline Ctry & an & 751 ‡a & Andorra & Name Term (NAF) \\
\hline Ctry & ao & 751 ‡a & Angola & Name Term (NAF) \\
\hline Ctry & $\mathrm{aq}$ & 751 ‡a & Antigua and Barbuda & Name Term (NAF) \\
\hline Ctry & aru & 751 ‡a & Arkansas & Name Term (NAF) \\
\hline Ctry & as & 751 ‡a & American Samoa & Name Term (NAF) \\
\hline Ctry & at & $751 \neq a$ & Australia & Name Term (NAF) \\
\hline Ctry & $\mathrm{au}$ & 751 ‡a & Austria & Name Term (NAF) \\
\hline Ctry & aw & 751 ‡a & Aruba & Name Term (NAF) \\
\hline Ctry & ay & 751 ‡a & Antarctica & Name Term (NAF) \\
\hline Ctry & azu & 751 ‡a & Arizona & Name Term (NAF) \\
\hline Ctry & ba & 751 ‡a & Bahrain & Name Term (NAF) \\
\hline
\end{tabular}


Conversion Table for the Fixed Field

\begin{tabular}{|c|c|c|c|c|}
\hline \multicolumn{2}{|c|}{ Fixed Field Value } & \multicolumn{2}{|c|}{ Variable Field Value } & \multirow{2}{*}{$\begin{array}{l}\text { Notes } \\
\text { Name Term (NAF) }\end{array}$} \\
\hline Ctry & $\mathrm{bb}$ & $751 \neq a$ & Barbados & \\
\hline Ctry & $\mathrm{bcc}$ & 751 ‡a & British Columbia & Name Term (NAF) \\
\hline Ctry & $\mathrm{bd}$ & $751 \neq a$ & Burundi & Name Term (NAF) \\
\hline Ctry & be & $751 \neq a$ & Belgium & Name Term (NAF) \\
\hline Ctry & bf & $751 \neq a$ & Bahamas & Name Term (NAF) \\
\hline Ctry & bg & 751 ‡a & Bangladesh & Name Term (NAF) \\
\hline Ctry & bh & $751 \neq a$ & Belize & Name Term (NAF) \\
\hline Ctry & bi & 751 ‡a & British Indian Ocean Territory & Name Term (NAF) \\
\hline Ctry & bl & $751 \neq a$ & Brazil & Name Term (NAF) \\
\hline Ctry & $\mathrm{bm}$ & 751 ła & Bermuda Islands & Name Term (NAF) \\
\hline Ctry & bn & $751 \neq a$ & Bosnia and Hercegovina & Name Term (NAF) \\
\hline Ctry & bo & $751 \neq a$ & Bolivia & Name Term (NAF) \\
\hline Ctry & $\mathrm{bp}$ & $751 \neq a$ & Solomon Islands & Name Term (NAF) \\
\hline Ctry & $\mathrm{br}$ & 751 ła & Burma & Name Term (NAF) \\
\hline Ctry & bs & 751 ‡a & Botswana & Name Term (NAF) \\
\hline Ctry & bt & 751 ła & Bhutan & Name Term (NAF) \\
\hline Ctry & bu & 751 ‡a & Bulgaria & Name Term (NAF) \\
\hline Ctry & bv & 751 ła & Bouvet Island & Name Term (NAF) \\
\hline Ctry & bw & 751 ‡a & Belarus & Name Term (NAF) \\
\hline Ctry & $-b w r$ & 751 ‡a & Byelorussian S.S.R. & Name Term (NAF) \\
\hline Ctry & bx & $751 \neq a$ & Brunei & Name Term (NAF) \\
\hline Ctry & ca & $751 \neq a$ & Caribbean Netherlands & Name Term (NAF) \\
\hline Ctry & cau & $751 \neq a$ & California & Name Term (NAF) \\
\hline Ctry & $\mathrm{cb}$ & $751 \neq a$ & Cambodia & Name Term (NAF) \\
\hline Ctry & cc & $751 \neq a$ & China & Name Term (NAF) \\
\hline Ctry & $\mathrm{cd}$ & $751 \neq a$ & Chad & Name Term (NAF) \\
\hline Ctry & ce & 751 ‡a & Sri Lanka & Name Term (NAF) \\
\hline Ctry & $\mathrm{cf}$ & $751 \neq a$ & Congo (Brazzaville) & Name Term (NAF) \\
\hline Ctry & $\mathrm{cg}$ & $751 \neq a$ & Congo (Democratic Republic) & Name Term (NAF) \\
\hline Ctry & $\mathrm{ch}$ & $751 \neq a$ & China (Republic : 1949- ) & Name Term (NAF) \\
\hline Ctry & $\mathrm{ci}$ & 751 ‡a & Croatia & Name Term (NAF) \\
\hline Ctry & cj & $751 \neq a$ & Cayman Islands & Name Term (NAF) \\
\hline Ctry & ck & 751 ‡a & Colombia & Name Term (NAF) \\
\hline Ctry & $\mathrm{cl}$ & $751 \neq a$ & Chile & Name Term (NAF) \\
\hline Ctry & $\mathrm{cm}$ & 751 ‡a & Cameroon & Name Term (NAF) \\
\hline Ctry & $-\mathrm{cn}$ & $751 \neq a$ & Canada & Name Term (NAF) \\
\hline Ctry & co & 751 ‡a & Curaçao & Name Term (NAF) \\
\hline Ctry & cou & $751 \neq a$ & Colorado & Name Term (NAF) \\
\hline Ctry & $-c p$ & 751 ła & Canton and Enderbury Islands & Name Term (NAF) \\
\hline Ctry & $\mathrm{cq}$ & $751 \neq a$ & Comoros & Name Term (NAF) \\
\hline Ctry & $\mathrm{cr}$ & 751 ła & Costa Rica & Name Term (NAF) \\
\hline Ctry & $-c s$ & $751 \neq a$ & Czechoslovakia & Name Term (NAF) \\
\hline Ctry & ctu & 751 ‡a & Connecticut & Name Term (NAF) \\
\hline Ctry & $\mathrm{cu}$ & $751 \neq a$ & Cuba & Name Term (NAF) \\
\hline Ctry & $\mathrm{cV}$ & 751 ła & Cape Verde & Name Term (NAF) \\
\hline Ctry & $\mathrm{cW}$ & $751 \neq a$ & Cook Islands & Name Term (NAF) \\
\hline Ctry & $c x$ & 751 ła & Central African Republic & Name Term (NAF) \\
\hline Ctry & cy & 751 ‡а & Cyprus & Name Term (NAF) \\
\hline
\end{tabular}


Conversion Table for the Fixed Field

\begin{tabular}{|c|c|c|c|c|}
\hline \multicolumn{2}{|c|}{ Fixed Field Value } & \multicolumn{2}{|c|}{ Variable Field Value } & \multirow{2}{*}{$\begin{array}{l}\text { Notes } \\
\text { Name Term (NAF) }\end{array}$} \\
\hline Ctry & $-c z$ & 751 ‡a & Canal Zone & \\
\hline Ctry & dcu & $751 \neq a$ & District of Columbia & Name Term (NAF) \\
\hline Ctry & deu & $751 \neq a$ & Delaware & Name Term (NAF) \\
\hline Ctry & $\mathrm{dk}$ & $751 \neq a$ & Denmark & Name Term (NAF) \\
\hline Ctry & $\mathrm{dm}$ & $751 \neq a$ & Benin & Name Term (NAF) \\
\hline Ctry & $\mathrm{dq}$ & $751 \neq a$ & Dominica & Name Term (NAF) \\
\hline Ctry & $\mathrm{dr}$ & $751 \neq a$ & Dominican Republic & Name Term (NAF) \\
\hline Ctry & ea & $751 \neq a$ & Eritrea & Name Term (NAF) \\
\hline Ctry & ec & $751 \neq a$ & Ecuador & Name Term (NAF) \\
\hline Ctry & eg & $751 \neq a$ & Equatorial Guinea & Name Term (NAF) \\
\hline Ctry & em & $751 \neq a$ & Timor-Leste & Name Term (NAF) \\
\hline Ctry & enk & $751 \neq a$ & England & Name Term (NAF) \\
\hline Ctry & er & $751 \neq a$ & Estonia & Name Term (NAF) \\
\hline Ctry & -err & $751 \neq a$ & Estonia & Name Term (NAF) \\
\hline Ctry & es & $751 \neq a$ & El Salvador & Name Term (NAF) \\
\hline Ctry & et & $751 \neq a$ & Ethiopia & Name Term (NAF) \\
\hline Ctry & fa & $751 \neq a$ & Faroe Islands & Name Term (NAF) \\
\hline Ctry & $\mathrm{fg}$ & $751 \neq a$ & French Guiana & Name Term (NAF) \\
\hline Ctry & $\mathrm{fi}$ & $751 \neq a$ & Finland & Name Term (NAF) \\
\hline Ctry & $f j$ & $751 \neq a$ & Fiji & Name Term (NAF) \\
\hline Ctry & $\mathrm{fk}$ & $751 \neq a$ & Falkland Islands & Name Term (NAF) \\
\hline Ctry & flu & $751 \neq a$ & Florida & Name Term (NAF) \\
\hline Ctry & $\mathrm{fm}$ & $751 \neq a$ & Micronesia (Federated States) & Name Term (NAF) \\
\hline Ctry & $\mathrm{fp}$ & $751 \neq a$ & French Polynesia & Name Term (NAF) \\
\hline Ctry & $\mathrm{fr}$ & $751 \neq a$ & France & Name Term (NAF) \\
\hline Ctry & fs & $751 \neq a$ & Terres australes et antarctiques françaises & Name Term (NAF) \\
\hline Ctry & $\mathrm{ft}$ & $751 \neq a$ & Djibouti & Name Term (NAF) \\
\hline Ctry & gau & $751 \neq a$ & Georgia & Name Term (NAF) \\
\hline Ctry & gb & $751 \neq a$ & Kiribati & Name Term (NAF) \\
\hline Ctry & gd & $751 \neq a$ & Grenada & Name Term (NAF) \\
\hline Ctry & -ge & $751 \neq a$ & Germany (East) & Name Term (NAF) \\
\hline Ctry & gh & $751 \neq a$ & Ghana & Name Term (NAF) \\
\hline Ctry & gi & $751 \neq a$ & Gibraltar & Name Term (NAF) \\
\hline Ctry & $\mathrm{gl}$ & $751 \neq a$ & Greenland & Name Term (NAF) \\
\hline Ctry & gm & $751 \neq a$ & Gambia & Name Term (NAF) \\
\hline Ctry & -gn & $751 \neq a$ & Gilbert and Ellice Islands & Name Term (NAF) \\
\hline Ctry & go & $751 \neq a$ & Gabon & Name Term (NAF) \\
\hline Ctry & gp & $751 \neq a$ & Guadeloupe & Name Term (NAF) \\
\hline Ctry & $\mathrm{gr}$ & $751 \neq a$ & Greece & Name Term (NAF) \\
\hline Ctry & gs & $751 \neq a$ & Georgia (Republic) & Name Term (NAF) \\
\hline Ctry & $-g s r$ & $751 \neq a$ & Georgian S.S.R. & Name Term (NAF) \\
\hline Ctry & gt & $751 \neq a$ & Guatemala & Name Term (NAF) \\
\hline Ctry & gu & $751 \neq a$ & Guam & Name Term (NAF) \\
\hline Ctry & gv & $751 \neq a$ & Guinea & Name Term (NAF) \\
\hline Ctry & gw & $751 \neq a$ & Germany & Name Term (NAF) \\
\hline Ctry & gy & $751 \neq a$ & Guyana & Name Term (NAF) \\
\hline Ctry & gz & $751 \neq a$ & Gaza Strip & Name Term (NAF) \\
\hline Ctry & hiu & $751 \neq a$ & Hawaii & Name Term (NAF) \\
\hline
\end{tabular}


Conversion Table for the Fixed Field

\begin{tabular}{|c|c|c|c|c|}
\hline \multicolumn{2}{|c|}{ Fixed Field Value } & \multicolumn{2}{|c|}{ Variable Field Value } & \multirow{2}{*}{$\begin{array}{l}\text { Notes } \\
\text { Name Term (NAF) }\end{array}$} \\
\hline Ctry & $-\mathrm{hk}$ & $751 \neq a$ & Hong Kong & \\
\hline Ctry & $\mathrm{hm}$ & $751 \neq a$ & Heard and McDonald Islands & Name Term (NAF) \\
\hline Ctry & ho & $751 \neq a$ & Honduras & Name Term (NAF) \\
\hline Ctry & ht & $751 \neq a$ & Haiti & Name Term (NAF) \\
\hline Ctry & hu & $751 \neq a$ & Hungary & Name Term (NAF) \\
\hline Ctry & iau & $751 \neq a$ & lowa & Name Term (NAF) \\
\hline Ctry & ic & $751 \neq a$ & Iceland & Name Term (NAF) \\
\hline Ctry & idu & $751 \neq a$ & Idaho & Name Term (NAF) \\
\hline Ctry & ie & $751 \neq a$ & Ireland & Name Term (NAF) \\
\hline Ctry & $\mathrm{ii}$ & $751 \neq a$ & India & Name Term (NAF) \\
\hline Ctry & ilu & $751 \neq a$ & Illinois & Name Term (NAF) \\
\hline Ctry & inu & $751 \neq a$ & Indiana & Name Term (NAF) \\
\hline Ctry & io & $751 \neq a$ & Indonesia & Name Term (NAF) \\
\hline Ctry & iq & $751 \neq a$ & Iraq & Name Term (NAF) \\
\hline Ctry & ir & $751 \neq a$ & Iran & Name Term (NAF) \\
\hline Ctry & is & $751 \neq a$ & Israel & Name Term (NAF) \\
\hline Ctry & it & $751 \neq a$ & Italy & Name Term (NAF) \\
\hline Ctry & -iu & $751 \neq a$ & Israel-Syria Demilitarized Zones & Name Term (NAF) \\
\hline Ctry & iv & $751 \neq a$ & Côte d'Ivoire & Name Term (NAF) \\
\hline Ctry & -iw & $751 \neq a$ & Israel-Jordan Demilitarized Zones & Name Term (NAF) \\
\hline Ctry & iy & $751 \neq a$ & Iraq-Saudi Arabia Neutral Zone & Name Term (NAF) \\
\hline Ctry & ja & $751 \neq a$ & Japan & Name Term (NAF) \\
\hline Ctry & $\mathrm{ji}$ & $751 \neq a$ & Johnston Atoll & Name Term (NAF) \\
\hline Ctry & jm & 751 ‡a & Jamaica & Name Term (NAF) \\
\hline Ctry & -jn & $751 \neq a$ & Jan Mayen & Name Term (NAF) \\
\hline Ctry & jo & 751 ‡a & Jordan & Name Term (NAF) \\
\hline Ctry & ke & $751 \neq a$ & Kenya & Name Term (NAF) \\
\hline Ctry & $\mathrm{kg}$ & $751 \neq a$ & Kyrgyzstan & Name Term (NAF) \\
\hline Ctry & $-k g r$ & $751 \neq a$ & Kirghiz S.S.R. & Name Term (NAF) \\
\hline Ctry & $\mathrm{kn}$ & $751 \neq a$ & Korea (North) & Name Term (NAF) \\
\hline Ctry & ko & $751 \neq a$ & Korea (South) & Name Term (NAF) \\
\hline Ctry & ksu & $751 \neq a$ & Kansas & Name Term (NAF) \\
\hline Ctry & $\mathrm{ku}$ & $751 \neq a$ & Kuwait & Name Term (NAF) \\
\hline Ctry & kv & $751 \neq a$ & Kosovo & Name Term (NAF) \\
\hline Ctry & kyu & $751 \neq a$ & Kentucky & Name Term (NAF) \\
\hline Ctry & $k z$ & $751 \neq a$ & Kazakhstan & Name Term (NAF) \\
\hline Ctry & $-k z r$ & $751 \neq a$ & Kazakh S.S.R. & Name Term (NAF) \\
\hline Ctry & lau & $751 \neq a$ & Louisiana & Name Term (NAF) \\
\hline Ctry & $\mathrm{lb}$ & $751 \neq a$ & Liberia & Name Term (NAF) \\
\hline Ctry & le & $751 \neq a$ & Lebanon & Name Term (NAF) \\
\hline Ctry & lh & $751 \neq a$ & Liechtenstein & Name Term (NAF) \\
\hline Ctry & li & $751 \neq a$ & Lithuania & Name Term (NAF) \\
\hline Ctry & -lir & $751 \neq a$ & Lithuania & Name Term (NAF) \\
\hline Ctry & $-\ln$ & $751 \neq a$ & Central and Southern Line Islands & Name Term (NAF) \\
\hline Ctry & lo & $751 \neq a$ & Lesotho & Name Term (NAF) \\
\hline Ctry & Is & $751 \neq a$ & Laos & Name Term (NAF) \\
\hline Ctry & lu & $751 \neq a$ & Luxembourg & Name Term (NAF) \\
\hline Ctry & Iv & $751 \neq a$ & Latvia & Name Term (NAF) \\
\hline
\end{tabular}


Conversion Table for the Fixed Field

\begin{tabular}{|c|c|c|c|c|}
\hline \multicolumn{2}{|c|}{ Fixed Field Value } & \multicolumn{2}{|c|}{ Variable Field Value } & \multirow{2}{*}{$\begin{array}{l}\text { Notes } \\
\text { Name Term (NAF) }\end{array}$} \\
\hline Ctry & $-\mid v r$ & 751 ‡a & Latvia & \\
\hline Ctry & ly & $751 \neq a$ & Libya & Name Term (NAF) \\
\hline Ctry & mau & $751 \ddagger a$ & Massachusetts & Name Term (NAF) \\
\hline Ctry & $\mathrm{mbc}$ & $751 \neq a$ & Manitoba & Name Term (NAF) \\
\hline Ctry & $\mathrm{mc}$ & $751 \ddagger a$ & Monaco & Name Term (NAF) \\
\hline Ctry & mdu & $751 \neq a$ & Maryland & Name Term (NAF) \\
\hline Ctry & meu & $751 \neq a$ & Maine & Name Term (NAF) \\
\hline Ctry & $\mathrm{mf}$ & $751 \ddagger a$ & Mauritius & Name Term (NAF) \\
\hline Ctry & $\mathrm{mg}$ & $751 \neq a$ & Madagascar & Name Term (NAF) \\
\hline Ctry & $-m h$ & 751 ‡a & Macao & Name Term (NAF) \\
\hline Ctry & miu & $751 \neq a$ & Michigan & Name Term (NAF) \\
\hline Ctry & $\mathrm{mj}$ & $751 \neq a$ & Montserrat & Name Term (NAF) \\
\hline Ctry & $\mathrm{mk}$ & $751 \neq a$ & Oman & Name Term (NAF) \\
\hline Ctry & $\mathrm{ml}$ & $751 \neq a$ & Mali & Name Term (NAF) \\
\hline Ctry & $\mathrm{mm}$ & $751 \neq a$ & Malta & Name Term (NAF) \\
\hline Ctry & $\mathrm{mnu}$ & $751 \neq a$ & Minnesota & Name Term (NAF) \\
\hline Ctry & mo & 751 ‡a & Montenegro & Name Term (NAF) \\
\hline Ctry & mou & 751 ‡a & Missouri & Name Term (NAF) \\
\hline Ctry & $\mathrm{mp}$ & $751 \neq a$ & Mongolia & Name Term (NAF) \\
\hline Ctry & $\mathrm{mq}$ & 751 ‡a & Martinique & Name Term (NAF) \\
\hline Ctry & $\mathrm{mr}$ & $751 \neq a$ & Morocco & Name Term (NAF) \\
\hline Ctry & $\mathrm{msu}$ & $751 \neq a$ & Mississippi & Name Term (NAF) \\
\hline Ctry & mtu & $751 \neq a$ & Montana & Name Term (NAF) \\
\hline Ctry & $\mathrm{mu}$ & $751 \neq a$ & Mauritania & Name Term (NAF) \\
\hline Ctry & $\mathrm{mv}$ & 751 ‡a & Moldova & Name Term (NAF) \\
\hline Ctry & $-m v r$ & 751 ‡a & Moldavian S.S.R. & Name Term (NAF) \\
\hline Ctry & $\mathrm{mw}$ & $751 \neq a$ & Malawi & Name Term (NAF) \\
\hline Ctry & $\mathrm{mx}$ & $751 \neq a$ & Mexico & Name Term (NAF) \\
\hline Ctry & my & $751 \ddagger a$ & Malaysia & Name Term (NAF) \\
\hline Ctry & $\mathrm{mz}$ & $751 \neq a$ & Mozambique & Name Term (NAF) \\
\hline Ctry & - na & $751 \ddagger a$ & Netherlands Antilles & Name Term (NAF) \\
\hline Ctry & $\mathrm{nbu}$ & $751 \neq a$ & Nebraska & Name Term (NAF) \\
\hline Ctry & ncu & $751 \neq a$ & North Carolina & Name Term (NAF) \\
\hline Ctry & ndu & $751 \neq a$ & North Dakota & Name Term (NAF) \\
\hline Ctry & ne & $751 \neq a$ & Netherlands & Name Term (NAF) \\
\hline Ctry & $\mathrm{nfc}$ & $751 \neq a$ & Newfoundland and Labrador & Name Term (NAF) \\
\hline Ctry & ng & $751 \neq a$ & Niger & Name Term (NAF) \\
\hline Ctry & nhu & $751 \neq a$ & New Hampshire & Name Term (NAF) \\
\hline Ctry & nik & $751 \neq a$ & Northern Ireland & Name Term (NAF) \\
\hline Ctry & nju & $751 \neq a$ & New Jersey & Name Term (NAF) \\
\hline Ctry & nkc & $751 \neq a$ & New Brunswick & Name Term (NAF) \\
\hline Ctry & $\mathrm{nl}$ & $751 \neq a$ & New Caledonia & Name Term (NAF) \\
\hline Ctry & $-\mathrm{nm}$ & $751 \neq a$ & Northern Mariana Islands & Name Term (NAF) \\
\hline Ctry & $\mathrm{nmu}$ & $751 \neq a$ & New Mexico & Name Term (NAF) \\
\hline Ctry & $\mathrm{nn}$ & $751 \neq a$ & Vanuatu & Name Term (NAF) \\
\hline Ctry & no & $751 \neq a$ & Norway & Name Term (NAF) \\
\hline Ctry & $\mathrm{np}$ & $751 \neq a$ & Nepal & Name Term (NAF) \\
\hline Ctry & $\mathrm{nq}$ & $751 \ddagger a$ & Nicaragua & Name Term (NAF) \\
\hline
\end{tabular}


Conversion Table for the Fixed Field

\begin{tabular}{|c|c|c|c|c|}
\hline \multicolumn{2}{|c|}{ Fixed Field Value } & \multicolumn{2}{|c|}{ Variable Field Value } & \multirow{2}{*}{$\begin{array}{l}\text { Notes } \\
\text { Name Term (NAF) }\end{array}$} \\
\hline Ctry & $\mathrm{nr}$ & $751 \neq a$ & Nigeria & \\
\hline Ctry & nsc & 751 ‡a & Nova Scotia & Name Term (NAF) \\
\hline Ctry & ntc & $751 \neq a$ & Northwest Territories & Name Term (NAF) \\
\hline Ctry & nu & $751 \neq a$ & Nauru & Name Term (NAF) \\
\hline Ctry & nuc & $751 \neq a$ & Nunavut & Name Term (NAF) \\
\hline Ctry & nvu & 751 ‡a & Nevada & Name Term (NAF) \\
\hline Ctry & nw & $751 \neq a$ & Northern Mariana Islands & Name Term (NAF) \\
\hline Ctry & $\mathrm{nx}$ & $751 \neq a$ & Norfolk Island & Name Term (NAF) \\
\hline Ctry & nyu & $751 \neq a$ & New York (State) & Name Term (NAF) \\
\hline Ctry & $\mathrm{nz}$ & 751 ‡a & New Zealand & Name Term (NAF) \\
\hline Ctry & ohu & $751 \neq a$ & Ohio & Name Term (NAF) \\
\hline Ctry & oku & 751 ła & Oklahoma & Name Term (NAF) \\
\hline Ctry & onc & 751 ‡a & Ontario & Name Term (NAF) \\
\hline Ctry & oru & 751 ła & Oregon & Name Term (NAF) \\
\hline Ctry & ot & 751 ‡a & Mayotte & Name Term (NAF) \\
\hline Ctry & pau & 751 ła & Pennsylvania & Name Term (NAF) \\
\hline Ctry & $\mathrm{pc}$ & $751 \neq a$ & Pitcairn Island & Name Term (NAF) \\
\hline Ctry & pe & 751 ła & Peru & Name Term (NAF) \\
\hline Ctry & $\mathrm{pf}$ & 751 ‡a & Paracel Islands & Name Term (NAF) \\
\hline Ctry & pg & $751 \neq a$ & Guinea-Bissau & Name Term (NAF) \\
\hline Ctry & $\mathrm{ph}$ & $751 \neq a$ & Philippines & Name Term (NAF) \\
\hline Ctry & pic & $751 \neq a$ & Prince Edward Island & Name Term (NAF) \\
\hline Ctry & pk & $751 \neq a$ & Pakistan & Name Term (NAF) \\
\hline Ctry & $\mathrm{pl}$ & $751 \neq a$ & Poland & Name Term (NAF) \\
\hline Ctry & $\mathrm{pn}$ & $751 \neq a$ & Panama & Name Term (NAF) \\
\hline Ctry & po & $751 \neq a$ & Portugal & Name Term (NAF) \\
\hline Ctry & $\mathrm{pp}$ & 751 ‡a & Papua New Guinea & Name Term (NAF) \\
\hline Ctry & $\mathrm{pr}$ & $751 \neq a$ & Puerto Rico & Name Term (NAF) \\
\hline Ctry & $-p t$ & $751 \neq a$ & Portuguese Timor & Name Term (NAF) \\
\hline Ctry & pw & $751 \neq a$ & Palau & Name Term (NAF) \\
\hline Ctry & py & 751 ‡a & Paraguay & Name Term (NAF) \\
\hline Ctry & qa & $751 \neq a$ & Qatar & Name Term (NAF) \\
\hline Ctry & qea & 751 ‡a & Queensland & Name Term (NAF) \\
\hline Ctry & quc & $751 \neq a$ & Québec (Province) & Name Term (NAF) \\
\hline Ctry & $\mathrm{rb}$ & 751 ‡a & Serbia & Name Term (NAF) \\
\hline Ctry & re & $751 \neq a$ & Réunion & Name Term (NAF) \\
\hline Ctry & rh & 751 ‡a & Zimbabwe & Name Term (NAF) \\
\hline Ctry & riu & $751 \neq a$ & Rhode Island & Name Term (NAF) \\
\hline Ctry & $\mathrm{rm}$ & 751 ła & Romania & Name Term (NAF) \\
\hline Ctry & ru & $751 \neq a$ & Russia (Federation) & Name Term (NAF) \\
\hline Ctry & -rur & 751 ła & Russian S.F.S.R. & Name Term (NAF) \\
\hline Ctry & rw & $751 \neq a$ & Rwanda & Name Term (NAF) \\
\hline Ctry & -ry & 751 ‡a & Ryukyu Islands, Southern & Name Term (NAF) \\
\hline Ctry & sa & $751 \neq a$ & South Africa & Name Term (NAF) \\
\hline Ctry & $-s b$ & 751 ła & Svalbard & Name Term (NAF) \\
\hline Ctry & sc & $751 \neq a$ & Saint-Barthélemy & Name Term (NAF) \\
\hline Ctry & scu & 751 ła & South Carolina & Name Term (NAF) \\
\hline Ctry & sd & 751 ‡а & South Sudan & Name Term (NAF) \\
\hline
\end{tabular}


Conversion Table for the Fixed Field

\begin{tabular}{|c|c|c|c|c|}
\hline \multicolumn{2}{|c|}{ Fixed Field Value } & \multicolumn{2}{|c|}{ Variable Field Value } & \multirow{2}{*}{$\begin{array}{l}\text { Notes } \\
\text { Name Term (NAF) }\end{array}$} \\
\hline Ctry & sdu & $751 \neq a$ & South Dakota & \\
\hline Ctry & se & $751 \neq a$ & Seychelles & Name Term (NAF) \\
\hline Ctry & sf & $751 \neq a$ & Sao Tome and Principe & Name Term (NAF) \\
\hline Ctry & sg & $751 \neq a$ & Senegal & Name Term (NAF) \\
\hline Ctry & sh & $751 \neq a$ & Spanish North Africa & Name Term (NAF) \\
\hline Ctry & si & $751 \neq a$ & Singapore & Name Term (NAF) \\
\hline Ctry & sj & $751 \neq a$ & Sudan & Name Term (NAF) \\
\hline Ctry & -sk & $751 \neq a$ & Sikkim & Name Term (NAF) \\
\hline Ctry & sl & $751 \neq a$ & Sierra Leone & Name Term (NAF) \\
\hline Ctry & $\mathrm{sm}$ & $751 \neq a$ & San Marino & Name Term (NAF) \\
\hline Ctry & sn & $751 \neq a$ & Sint Maarten & Name Term (NAF) \\
\hline Ctry & snc & $751 \neq a$ & Saskatchewan & Name Term (NAF) \\
\hline Ctry & so & $751 \neq a$ & Somalia & Name Term (NAF) \\
\hline Ctry & $s p$ & $751 \neq a$ & Spain & Name Term (NAF) \\
\hline Ctry & sq & $751 \neq a$ & Swaziland & Name Term (NAF) \\
\hline Ctry & $s r$ & $751 \neq a$ & Surinam & Name Term (NAF) \\
\hline Ctry & ss & $751 \neq a$ & Western Sahara & Name Term (NAF) \\
\hline Ctry & st & $751 \neq a$ & Saint-Martin & Name Term (NAF) \\
\hline Ctry & stk & $751 \neq a$ & Scotland & Name Term (NAF) \\
\hline Ctry & su & $751 \neq a$ & Saudi Arabia & Name Term (NAF) \\
\hline Ctry & -sv & $751 \neq a$ & Swan Islands & Name Term (NAF) \\
\hline Ctry & sw & $751 \neq a$ & Sweden & Name Term (NAF) \\
\hline Ctry & sx & $751 \neq a$ & Namibia & Name Term (NAF) \\
\hline Ctry & sy & 751 ‡a & Syria & Name Term (NAF) \\
\hline Ctry & sz & $751 \neq a$ & Switzerland & Name Term (NAF) \\
\hline Ctry & ta & $751 \neq a$ & Tajikistan & Name Term (NAF) \\
\hline Ctry & $-\operatorname{tar}$ & $751 \neq a$ & Tajik S.S.R. & Name Term (NAF) \\
\hline Ctry & tc & $751 \neq a$ & Turks and Caicos Islands & Name Term (NAF) \\
\hline Ctry & $\operatorname{tg}$ & $751 \neq a$ & Togo & Name Term (NAF) \\
\hline Ctry & th & $751 \neq a$ & Thailand & Name Term (NAF) \\
\hline Ctry & $\mathrm{ti}$ & $751 \neq a$ & Tunisia & Name Term (NAF) \\
\hline Ctry & tk & $751 \neq a$ & Turkmenistan & Name Term (NAF) \\
\hline Ctry & $-\mathrm{tkr}$ & $751 \neq a$ & Turkmen S.S.R. & Name Term (NAF) \\
\hline Ctry & $\mathrm{tl}$ & $751 \neq a$ & Tokelau & Name Term (NAF) \\
\hline Ctry & tma & $751 \neq a$ & Tasmania & Name Term (NAF) \\
\hline Ctry & tnu & $751 \neq a$ & Tennessee & Name Term (NAF) \\
\hline Ctry & to & $751 \neq a$ & Tonga & Name Term (NAF) \\
\hline Ctry & $\operatorname{tr}$ & $751 \neq a$ & Trinidad and Tobago & Name Term (NAF) \\
\hline Ctry & ts & $751 \neq a$ & United Arab Emirates & Name Term (NAF) \\
\hline Ctry & $-\mathrm{tt}$ & $751 \neq a$ & Trust Territory of the Pacific Islands & Name Term (NAF) \\
\hline Ctry & tu & $751 \neq a$ & Turkey & Name Term (NAF) \\
\hline Ctry & tv & $751 \neq a$ & Tuvalu & Name Term (NAF) \\
\hline Ctry & txu & $751 \neq a$ & Texas & Name Term (NAF) \\
\hline Ctry & $\mathrm{tz}$ & $751 \neq a$ & Tanzania & Name Term (NAF) \\
\hline Ctry & ua & $751 \neq a$ & Egypt & Name Term (NAF) \\
\hline Ctry & uc & $751 \neq a$ & United States Misc. Caribbean Islands & Name Term (NAF) \\
\hline Ctry & ug & $751 \neq a$ & Uganda & Name Term (NAF) \\
\hline Ctry & -ui & $751 \neq a$ & United Kingdom Misc. Islands & Name Term (NAF) \\
\hline
\end{tabular}


Conversion Table for the Fixed Field

\begin{tabular}{|c|c|c|c|c|}
\hline \multicolumn{2}{|c|}{ Fixed Field Value } & \multicolumn{2}{|c|}{ Variable Field Value } & \multirow{2}{*}{$\begin{array}{l}\text { Notes } \\
\text { Name Term (NAF) }\end{array}$} \\
\hline Ctry & uik & $751 \neq a$ & United Kingdom Misc. Islands & \\
\hline Ctry & -uk & $751 \neq a$ & United Kingdom & Name Term (NAF) \\
\hline Ctry & un & $751 \neq a$ & Ukraine & Name Term (NAF) \\
\hline Ctry & -unr & $751 \neq a$ & Ukraine & Name Term (NAF) \\
\hline Ctry & up & $751 \neq a$ & United States Misc. Pacific Islands & Name Term (NAF) \\
\hline Ctry & -ur & 751 ‡a & Soviet Union & Name Term (NAF) \\
\hline Ctry & -us & $751 \neq a$ & United States & Name Term (NAF) \\
\hline Ctry & utu & 751 ‡a & Utah & Name Term (NAF) \\
\hline Ctry & uv & $751 \neq a$ & Burkina Faso & Name Term (NAF) \\
\hline Ctry & uy & $751 \neq a$ & Uruguay & Name Term (NAF) \\
\hline Ctry & uz & $751 \neq a$ & Uzbekistan & Name Term (NAF) \\
\hline Ctry & $-u z r$ & $751 \neq a$ & Uzbek S.S.R. & Name Term (NAF) \\
\hline Ctry & vau & $751 \neq a$ & Virginia & Name Term (NAF) \\
\hline Ctry & $\mathrm{vb}$ & $751 \neq a$ & British Virgin Islands & Name Term (NAF) \\
\hline Ctry & vc & $751 \neq a$ & Vatican City & Name Term (NAF) \\
\hline Ctry & ve & $751 \neq a$ & Venezuela & Name Term (NAF) \\
\hline Ctry & vi & $751 \neq a$ & Virgin Islands of the United States & Name Term (NAF) \\
\hline Ctry & $\mathrm{vm}$ & $751 \neq a$ & Vietnam & Name Term (NAF) \\
\hline Ctry & $-\mathrm{vn}$ & $751 \neq a$ & Vietnam, North & Name Term (NAF) \\
\hline Ctry & $\mathrm{vp}$ & $751 \neq a$ & Various places & Name Term (NAF) \\
\hline Ctry & vra & $751 \neq a$ & Victoria & Name Term (NAF) \\
\hline Ctry & -vs & $751 \neq a$ & Vietnam, South & Name Term (NAF) \\
\hline Ctry & vtu & $751 \neq a$ & Vermont & Name Term (NAF) \\
\hline Ctry & wau & $751 \neq a$ & Washington (State) & Name Term (NAF) \\
\hline Ctry & $-w b$ & $751 \neq a$ & West Berlin & Name Term (NAF) \\
\hline Ctry & wea & $751 \neq a$ & Western Australia & Name Term (NAF) \\
\hline Ctry & $w f$ & $751 \neq a$ & Wallis and Futuna & Name Term (NAF) \\
\hline Ctry & wiu & $751 \neq a$ & Wisconsin & Name Term (NAF) \\
\hline Ctry & wj & $751 \neq a$ & West Bank of the Jordan River & Name Term (NAF) \\
\hline Ctry & wk & $751 \neq a$ & Wake Island & Name Term (NAF) \\
\hline Ctry & wlk & 751 ‡a & Wales & Name Term (NAF) \\
\hline Ctry & ws & $751 \neq a$ & Samoa & Name Term (NAF) \\
\hline Ctry & wvu & 751 ‡a & West Virginia & Name Term (NAF) \\
\hline Ctry & wyu & $751 \neq a$ & Wyoming & Name Term (NAF) \\
\hline Ctry & xa & $751 \neq a$ & Christmas Island (Indian Ocean) & Name Term (NAF) \\
\hline Ctry & $x b$ & $751 \neq a$ & Cocos (Keeling) Islands & Name Term (NAF) \\
\hline Ctry & $x c$ & $751 \neq a$ & Maldives & Name Term (NAF) \\
\hline Ctry & $x d$ & $751 \neq a$ & Saint Kitts-Nevis & Name Term (NAF) \\
\hline Ctry & xe & $751 \neq a$ & Marshall Islands & Name Term (NAF) \\
\hline Ctry & $x f$ & $751 \neq a$ & Midway Islands & Name Term (NAF) \\
\hline Ctry & xga & $751 \neq a$ & Coral Sea Islands Territory & Name Term (NAF) \\
\hline Ctry & $\mathrm{xh}$ & $751 \neq a$ & Niue & Name Term (NAF) \\
\hline Ctry & $-x i$ & $751 \neq a$ & Saint Kitts-Nevis-Anguilla & Name Term (NAF) \\
\hline Ctry & $x j$ & $751 \neq a$ & Saint Helena & Name Term (NAF) \\
\hline Ctry & $\mathrm{xk}$ & $751 \neq a$ & Saint Lucia & Name Term (NAF) \\
\hline Ctry & $\mathrm{xl}$ & $751 \neq a$ & Saint Pierre and Miquelon & Name Term (NAF) \\
\hline Ctry & $\mathrm{xm}$ & $751 \neq a$ & Saint Vincent and the Grenadines & Name Term (NAF) \\
\hline Ctry & $\mathrm{xn}$ & $751 \neq a$ & Macedonia & Name Term (NAF) \\
\hline
\end{tabular}


Conversion Table for the Fixed Field

\begin{tabular}{|c|c|c|c|c|}
\hline \multicolumn{2}{|c|}{ Fixed Field Value } & \multicolumn{2}{|c|}{ Variable Field Value } & \multirow{2}{*}{$\begin{array}{l}\text { Notes } \\
\text { Name Term (NAF) }\end{array}$} \\
\hline Ctry & xna & 751 ‡a & New South Wales & \\
\hline Ctry & xo & 751 ‡a & Slovakia & Name Term (NAF) \\
\hline Ctry & xoa & $751 \neq a$ & Northern Territory & Name Term (NAF) \\
\hline Ctry & $x p$ & $751 \neq a$ & Spratly Island & Name Term (NAF) \\
\hline Ctry & $\mathrm{xr}$ & $751 \neq a$ & Czech Republic & Name Term (NAF) \\
\hline Ctry & xra & $751 \neq a$ & South Australia & Name Term (NAF) \\
\hline Ctry & xs & $751 \neq a$ & South Georgia and the South Sandwich & Name Term (NAF) \\
\hline Ctry & $x v$ & $751 \neq a$ & Slovenia & Name Term (NAF) \\
\hline Ctry & $x x$ & & & \\
\hline Ctry & $x x c$ & $751 \neq a$ & Canada & Name Term (NAF) \\
\hline Ctry & $\mathrm{xxk}$ & $751 \neq a$ & United Kingdom & Name Term (NAF) \\
\hline Ctry & $-\mathrm{xxr}$ & $751 \neq a$ & Soviet Union & Name Term (NAF) \\
\hline Ctry & $\mathrm{xxu}$ & $751 \neq a$ & United States & Name Term (NAF) \\
\hline Ctry & ye & $751 \neq a$ & Yemen & Name Term (NAF) \\
\hline Ctry & ykc & $751 \neq a$ & Yukon Territory & Name Term (NAF) \\
\hline Ctry & -ys & $751 \neq a$ & Yemen (People's Democratic Republic) & Name Term (NAF) \\
\hline Ctry & $-\mathrm{yu}$ & $751 \neq a$ & Serbia and Montenegro & Name Term (NAF) \\
\hline Ctry & za & $751 \neq a$ & Zambia & Name Term (NAF) \\
\hline Date 1 & <yуyy> & & & No equivalent element \\
\hline Date 2 & <yyyy> & & & No equivalent element \\
\hline Desc & <blank> & & & Administrative Metadata \\
\hline Desc & $\mathrm{a}$ & & & Administrative Metadata \\
\hline Desc & C & & & Administrative Metadata \\
\hline Desc & $\mathrm{i}$ & & & Administrative Metadata \\
\hline Desc & $\mathrm{u}$ & & & Administrative Metadata \\
\hline$\overline{\mathrm{DtSt}}$ & $<$ blank> & & & No equivalent element \\
\hline DtSt & $\mathrm{b}$ & & & No equivalent element \\
\hline DtSt & c & & & No equivalent element \\
\hline DtSt & $d$ & & & No equivalent element \\
\hline DtSt & $\mathrm{e}$ & & & No equivalent element \\
\hline DtSt & $\mathrm{i}$ & & & No equivalent element \\
\hline DtSt & $\mathrm{k}$ & & & No equivalent element \\
\hline DtSt & $\mathrm{m}$ & & & No equivalent element \\
\hline DtSt & $\mathrm{n}$ & & & No equivalent element \\
\hline DtSt & $\mathrm{p}$ & & & No equivalent element \\
\hline DtSt & $q$ & & & No equivalent element \\
\hline DtSt & $r$ & & & No equivalent element \\
\hline DtSt & $\mathrm{s}$ & & & No equivalent element \\
\hline DtSt & $\mathrm{t}$ & & & No equivalent element \\
\hline DtSt & $\mathrm{u}$ & & & No equivalent element \\
\hline Elvl & <blank> & & & Administrative Metadata \\
\hline Elvl & 1 & & & Administrative Metadata \\
\hline Elvl & 2 & & & Administrative Metadata \\
\hline Elvl & 3 & & & Administrative Metadata \\
\hline Elvl & 4 & & & Administrative Metadata \\
\hline Elvl & 5 & & & Administrative Metadata \\
\hline Elvl & 7 & & & Administrative Metadata \\
\hline Elvl & 8 & & & Administrative Metadata \\
\hline
\end{tabular}


Conversion Table for the Fixed Field

\begin{tabular}{|c|c|c|c|c|}
\hline \multicolumn{2}{|c|}{ Fixed Field Value } & \multicolumn{2}{|c|}{ Variable Field Value } & \multirow{2}{*}{$\begin{array}{l}\text { Notes } \\
\text { Administrative Metadata }\end{array}$} \\
\hline Elvl & $\mathrm{i}$ & & & \\
\hline Elvl & $\mathrm{k}$ & & & Administrative Metadata \\
\hline Elvl & I & & & Administrative Metadata \\
\hline Elvl & $\mathrm{m}$ & & & Administrative Metadata \\
\hline Elvl & $\mathrm{e}$ & & & Administrative Metadata \\
\hline Elvl & j & & & Administrative Metadata \\
\hline Entered & \multicolumn{3}{|c|}{ <yyyymmdd> } & Administrative Metadata \\
\hline EntW & \multicolumn{3}{|c|}{$<$ blank> } & No equivalent value \\
\hline EntW & $\mathrm{a}$ & 655 ‡a & Abstracts & Genre Term (LCGFT) \\
\hline Entw & $\mathrm{b}$ & $655 \neq a$ & Bibliographies & Genre Term (LCGFT) \\
\hline EntW & c & $655 \neq a$ & Catalogs & Genre Term (LCGFT) \\
\hline Entw & $d$ & $655 \neq a$ & Dictionaries & Genre Term (LCGFT) \\
\hline EntW & $\mathrm{e}$ & $655 \neq a$ & Encyclopedias & Genre Term (LCGFT) \\
\hline EntW & $f$ & $655 \neq a$ & Handbooks and manuals & Genre Term (LCGFT) \\
\hline EntW & $\mathrm{g}$ & 655 ‡a & Law reviews & Genre Term (LCGFT) \\
\hline EntW & $\mathrm{h}$ & $655 \neq a$ & Biographies & Genre Term (LCGFT) \\
\hline EntW & $\mathrm{i}$ & 655 ‡a & Indexes & Genre Term (LCGFT) \\
\hline EntW & $\mathrm{k}$ & $655 \neq a$ & Discographies & Genre Term (LCGFT) \\
\hline EntW & I & 655 ‡a & Statutes and codes & Genre Term (LCGFT) \\
\hline EntW & $\mathrm{m}$ & $655 \neq a$ & Academic theses & Genre Term (LCGFT) \\
\hline Entw & $\mathrm{n}$ & & & No equivalent value \\
\hline EntW & 0 & $655 \neq a$ & Reviews & Genre Term (LCGFT) \\
\hline EntW & $\mathrm{p}$ & $655 \neq a$ & Programmed instructional materials & Genre Term (LCGFT) \\
\hline EntW & $\mathrm{q}$ & & & No equivalent value \\
\hline EntW & $r$ & $655 \neq a$ & Directories & Genre Term (LCGFT) \\
\hline EntW & $\mathrm{s}$ & $655 \neq a$ & Statistics & Genre Term (LCGFT) \\
\hline EntW & $\mathrm{t}$ & & & No equivalent value \\
\hline EntW & u & & & No equivalent value \\
\hline EntW & $\mathrm{v}$ & $655 \neq a$ & Casebooks & Genre Term (LCGFT) \\
\hline EntW & w & $655 \neq a$ & Court decisions and opinions & Genre Term (LCGFT) \\
\hline EntW & $\mathrm{y}$ & 655 ‡a & Yearbooks & Genre Term (LCGFT) \\
\hline EntW & z & $655 \neq a$ & Treaties & Genre Term (LCGFT) \\
\hline EntW & 5 & $655 \neq a$ & Calendars & Genre Term (LCGFT) \\
\hline EntW & 6 & $655 \neq a$ & Comics (Graphic works) & Genre Term (LCGFT) \\
\hline Fest & 0 & & & No equivalent value \\
\hline Fest & 1 & $700 \ddagger \mathrm{e}$ & honouree & Must be done manually \\
\hline$\overline{\text { File }}$ & $a$ & $347 \neq a$ & data file & \\
\hline File & $b$ & $347 \ddagger a$ & program file & \\
\hline File & c & $347 \neq a$ & image file & \\
\hline File & $d$ & $347 \neq a$ & text file & \\
\hline \multirow[t]{2}{*}{ File } & $\mathrm{e}$ & 347 ‡a & data file & \\
\hline & \multicolumn{2}{|c|}{ and... 655 ‡a } & Bibliographies & Genre Term (LCGFT) \\
\hline File & $f$ & $347 \ddagger a$ & typography file & Proposed RDA value \\
\hline \multirow[t]{2}{*}{ File } & \multirow[t]{2}{*}{$\mathrm{g}$} & $347 \ddagger a$ & program file & \\
\hline & & $6 X X \mp v$ & Computer games & Subject Term (LCSH) \\
\hline File & $\mathrm{h}$ & $347 \ddagger a$ & audio file & \\
\hline File & $\mathrm{i}$ & & & No equivalent value \\
\hline File & j & $338 \neq a$ & online resource & Do not migrate \\
\hline
\end{tabular}


Conversion Table for the Fixed Field

\begin{tabular}{|c|c|c|c|c|}
\hline \multicolumn{2}{|c|}{ Fixed Field Value } & \multicolumn{2}{|c|}{ Variable Field Value } & \multirow{2}{*}{$\begin{array}{l}\text { Notes } \\
\text { No equivalent value }\end{array}$} \\
\hline File & $\mathrm{m}$ & & & \\
\hline File & u & & & No equivalent value \\
\hline File & $z$ & & & No equivalent value \\
\hline Fmus & $\mathrm{a}$ & $254 \ddagger a$ & Score & \\
\hline Fmus & $\mathrm{b}$ & $254 \neq a$ & Full score, miniature or study size & Proposed RDA value \\
\hline Fmus & c & $254 \neq a$ & Accompaniment reduced for keyboard & Proposed RDA value \\
\hline Fmus & $d$ & $254 \neq a$ & Voice score with accompaniment omitted & Proposed RDA value \\
\hline Fmus & e & $254 \neq a$ & Condensed score or piano-conductor score & Proposed RDA value \\
\hline Fmus & g & $254 \neq a$ & Close score & Proposed RDA value \\
\hline Fmus & $\mathrm{h}$ & $254 \ddagger a$ & Chorus score & \\
\hline Fmus & $\mathrm{i}$ & $254 \neq a$ & Condensed score & \\
\hline Fmus & j & $254 \neq a$ & Performer-conductor part & Proposed RDA value \\
\hline Fmus & $\mathrm{k}$ & $254 \neq a$ & Vocal score & \\
\hline Fmus & $\mathrm{m}$ & & & No equivalent value \\
\hline Fmus & $\mathrm{n}$ & & & No equivalent value \\
\hline Fmus & $\mathrm{u}$ & & & No equivalent value \\
\hline Fmus & z & & & No equivalent value \\
\hline Form & <blank> & & & No equivalent value \\
\hline Form & a & $338 \neq a$ & $\begin{array}{l}\text { microfilm cartridge/microfilm } \\
\text { cassette/microfilm reel/microfilm } \\
\text { roll/microfilm slip }\end{array}$ & Split values; Do not migrate \\
\hline Form & $\mathrm{b}$ & $338 \mp a$ & microfiche & Do not migrate \\
\hline Form & c & $338 \neq a$ & microopaque & Do not migrate \\
\hline Form & $d$ & $340 \mp n$ & large print & \\
\hline Form & $f$ & $546 \neq b$ & braille code & \\
\hline Form & 0 & $338 \neq a$ & online resource & Do not migrate \\
\hline Form & $q$ & $338 \neq a$ & $\begin{array}{l}\text { computer card/computer chip } \\
\text { cartridge/computer disc/computer disc } \\
\text { cartridge/computer tape cartridge/computer } \\
\text { tape cassette/computer tape reel }\end{array}$ & Split values; Do not migrate \\
\hline
\end{tabular}

\begin{tabular}{|c|c|c|c|c|}
\hline \multirow{2}{*}{$\begin{array}{l}\text { Form } \\
\text { Form }\end{array}$} & \multicolumn{3}{|l|}{$r$} & \multirow[t]{2}{*}{ No equivalent value } \\
\hline & $s$ & $337 \ddagger a$ & computer & \\
\hline Freq & $\overline{-}$ & & & No equivalent value \\
\hline Freq & $<$ blank> & $310 \neq a$ & irregular & \\
\hline Freq & $\mathrm{a}$ & $310 \neq a$ & annual & \\
\hline Freq & $\mathrm{b}$ & $310 \neq a$ & bimonthly & \\
\hline Freq & c & $310 \neq a$ & semiweekly & \\
\hline Freq & $d$ & $310 \neq a$ & daily & \\
\hline Freq & $\mathrm{e}$ & $310 \neq a$ & biweekly & \\
\hline Freq & $f$ & $310 \neq a$ & semiannual & \\
\hline Freq & g & $310 \neq a$ & biennial & \\
\hline Freq & $\mathrm{h}$ & $310 \neq a$ & triennial & \\
\hline Freq & $\mathrm{i}$ & $310 \neq a$ & three times a week & \\
\hline Freq & j & $310 \neq a$ & three times a month & \\
\hline Freq & $\mathrm{k}$ & $310 \ddagger a$ & continuously updated & Proposed RDA value \\
\hline Freq & $\mathrm{m}$ & $310 \neq a$ & monthly & \\
\hline Freq & $q$ & $310 \neq a$ & quarterly & \\
\hline
\end{tabular}


Conversion Table for the Fixed Field

\begin{tabular}{|c|c|c|c|c|}
\hline \multicolumn{2}{|c|}{ Fixed Field Value } & \multicolumn{2}{|c|}{ Variable Field Value } & \multirow[t]{2}{*}{ Notes } \\
\hline Freq & s & $310 \neq a$ & semimonthly & \\
\hline Freq & $\mathrm{t}$ & 310 ‡a & three times a year & \\
\hline Freq & $\mathrm{u}$ & & & No equivalent value \\
\hline Freq & w & $310 \neq a$ & weekly & \\
\hline Freq & z & & & No equivalent value \\
\hline Gpub & $<$ blank> & & & No equivalent element \\
\hline Gpub & $\mathrm{a}$ & & & No equivalent element \\
\hline Gpub & c & & & No equivalent element \\
\hline Gpub & $f$ & & & No equivalent element \\
\hline Gpub & $\mathrm{i}$ & & & No equivalent element \\
\hline Gpub & I & & & No equivalent element \\
\hline Gpub & $\mathrm{m}$ & & & No equivalent element \\
\hline Gpub & 0 & & & No equivalent element \\
\hline Gpub & s & & & No equivalent element \\
\hline Gpub & $\mathrm{u}$ & & & No equivalent element \\
\hline Gpub & z & & & No equivalent element \\
\hline Ills 1 & <blank> & & & No equivalent value \\
\hline Ills 1 & $\mathrm{a}$ & $300 \ddagger b$ & illustrations & Concatenation required \\
\hline Ills 1 & $\mathrm{~b}$ & $300 \ddagger b$ & maps & Concatenation required \\
\hline Ills 1 & c & $300 \ddagger b$ & portraits & Concatenation required \\
\hline Ills 1 & $d$ & $300 \ddagger b$ & charts & Concatenation required \\
\hline Ills 1 & $\mathrm{e}$ & $300 \ddagger b$ & plans & Concatenation required \\
\hline Ills 1 & $f$ & $300 \ddagger b$ & plates & Proposed RDA value; Concatenation required \\
\hline Ills 1 & g & $300 \neq b$ & music & Concatenation required \\
\hline Ills 1 & $\mathrm{~h}$ & $300 \ddagger b$ & facsimiles & Concatenation required \\
\hline Ills 1 & $\mathrm{i}$ & $300 \ddagger b$ & coats of arms & Concatenation required \\
\hline Ills 1 & j & $300 \ddagger b$ & genealogical tables & Concatenation required \\
\hline Ills 1 & $\mathrm{k}$ & $300 \ddagger b$ & forms & Concatenation required \\
\hline Ills 1 & I & $300 \ddagger b$ & samples & Concatenation required \\
\hline Ills 1 & $\mathrm{~m}$ & $300 \ddagger b$ & & No equivalent value \\
\hline Ills 1 & 0 & $300 \ddagger b$ & photographs & Concatenation required \\
\hline Ills 1 & $\mathrm{p}$ & $300 \ddagger b$ & illuminations & Concatenation required \\
\hline Ills 2 & <blank> & & & No equivalent value \\
\hline IIIs 2 & $\mathrm{a}$ & $300 \ddagger b$ & illustrations & Concatenation required \\
\hline Ills 2 & $\mathrm{~b}$ & $300 \ddagger b$ & maps & Concatenation required \\
\hline IIIs 2 & $c$ & $300 \ddagger b$ & portraits & Concatenation required \\
\hline Ills 2 & $d$ & $300 \ddagger b$ & charts & Concatenation required \\
\hline IIls 2 & $\mathrm{e}$ & $300 \ddagger b$ & plans & Concatenation required \\
\hline Ills 2 & $f$ & $300 \ddagger b$ & plates & Proposed RDA value; Concatenation required \\
\hline Ills 2 & g & $300 \ddagger b$ & music & Concatenation required \\
\hline Ills 2 & $\mathrm{~h}$ & $300 \ddagger b$ & facsimiles & Concatenation required \\
\hline IIIs 2 & $\mathrm{i}$ & $300 \ddagger b$ & coats of arms & Concatenation required \\
\hline Ills 2 & j & $300 \ddagger b$ & genealogical tables & Concatenation required \\
\hline IIIs 2 & k & $300 \ddagger b$ & forms & Concatenation required \\
\hline IIIs 2 & 1 & $300 \ddagger b$ & samples & Concatenation required \\
\hline IIls 2 & $\mathrm{~m}$ & $300 \ddagger b$ & & No equivalent value \\
\hline IIls 2 & 0 & $300 \ddagger b$ & photographs & Concatenation required \\
\hline Ills 2 & $\mathrm{p}$ & $300 \ddagger b$ & illuminations & Concatenation required \\
\hline
\end{tabular}




\section{Conversion Table for the Fixed Field}

\begin{tabular}{|c|c|c|c|c|}
\hline \multicolumn{2}{|c|}{ Fixed Field Value } & \multicolumn{2}{|c|}{ Variable Field Value } & \multirow{2}{*}{$\begin{array}{l}\text { Notes } \\
\text { No equivalent value }\end{array}$} \\
\hline Ills 3 & $<$ blank> & & & \\
\hline Ills 3 & $\mathrm{a}$ & $300 \ddagger b$ & illustrations & Concatenation required \\
\hline Ills 3 & $\mathrm{~b}$ & $300 \ddagger b$ & maps & Concatenation required \\
\hline Ills 3 & c & $300 \ddagger b$ & portraits & Concatenation required \\
\hline Ills 3 & $d$ & $300 \neq b$ & charts & Concatenation required \\
\hline Ills 3 & $\mathrm{e}$ & $300 \ddagger b$ & plans & Concatenation required \\
\hline Ills 3 & $f$ & $300 \ddagger b$ & plates & Proposed RDA value; Concatenation required \\
\hline Ills 3 & g & $300 \neq b$ & music & Concatenation required \\
\hline Ills 3 & $\mathrm{~h}$ & $300 \ddagger b$ & facsimiles & Concatenation required \\
\hline Ills 3 & $\mathrm{i}$ & $300 \neq b$ & coats of arms & Concatenation required \\
\hline Ills 3 & j & $300 \ddagger b$ & genealogical tables & Concatenation required \\
\hline Ills 3 & $\mathrm{k}$ & $300 \mp b$ & forms & Concatenation required \\
\hline Ills 3 & I & $300 \mp b$ & samples & Concatenation required \\
\hline Ills 3 & $\mathrm{~m}$ & $300 \neq b$ & & No equivalent value \\
\hline Ills 3 & 0 & $300 \mp b$ & photographs & Concatenation required \\
\hline Ills 3 & $\mathrm{p}$ & $300 \neq b$ & illuminations & Concatenation required \\
\hline Ills 4 & $<$ blank $>$ & & & No equivalent value \\
\hline Ills 4 & $a$ & $300 \neq b$ & illustrations & Concatenation required \\
\hline Ills 4 & $b$ & $300 \mp b$ & maps & Concatenation required \\
\hline Ills 4 & c & $300 \mp b$ & portraits & Concatenation required \\
\hline Ills 4 & $d$ & $300 \mp b$ & charts & Concatenation required \\
\hline Ills 4 & $\mathrm{e}$ & $300 \mp b$ & plans & Concatenation required \\
\hline Ills 4 & $f$ & $300 \mp b$ & plates & Proposed RDA value; Concatenation required \\
\hline Ills 4 & g & $300 \neq b$ & music & Concatenation required \\
\hline Ills 4 & $\mathrm{~h}$ & $300 \mp b$ & facsimiles & Concatenation required \\
\hline Ills 4 & $\mathrm{i}$ & $300 \neq b$ & coats of arms & Concatenation required \\
\hline Ills 4 & j & $300 \mp b$ & genealogical tables & Concatenation required \\
\hline Ills 4 & $\mathrm{k}$ & $300 \mp b$ & forms & Concatenation required \\
\hline Ills 4 & I & $300 \neq b$ & samples & Concatenation required \\
\hline Ills 4 & $\mathrm{~m}$ & $300 \mp b$ & & No equivalent value \\
\hline Ills 4 & 0 & $300 \neq b$ & photographs & Concatenation required \\
\hline Ills 4 & $\mathrm{p}$ & $300 \neq b$ & illuminations & Concatenation required \\
\hline$\overline{l n d x}$ & 0 & & & No equivalent value \\
\hline $\operatorname{lndx}$ & 1 & $504 \neq a$ & Includes index & \\
\hline Lang & aar & $377 \mp 1$ & Afar & MARC Language Term \\
\hline Lang & abk & $377 \ddagger 1$ & Abkhaz & MARC Language Term \\
\hline Lang & ace & $377 \ddagger 1$ & Achinese & MARC Language Term \\
\hline Lang & ach & $377 \ddagger 1$ & Acoli & MARC Language Term \\
\hline Lang & ada & $377 \ddagger 1$ & Adangme & MARC Language Term \\
\hline Lang & ady & $377 \ddagger 1$ & Adygei & MARC Language Term \\
\hline Lang & afa & $377 \ddagger 1$ & Afroasiatic (Other) & MARC Language Term \\
\hline Lang & afh & $377 \ddagger 1$ & Afrihili (Artificial language) & MARC Language Term \\
\hline Lang & afr & $377 \ddagger 1$ & Afrikaans & MARC Language Term \\
\hline Lang & ain & $377 \ddagger 1$ & Ainu & MARC Language Term \\
\hline Lang & -ajm & $377 \ddagger 1$ & Aljamía & MARC Language Term \\
\hline Lang & aka & $377 \ddagger 1$ & Akan & MARC Language Term \\
\hline Lang & akk & $377 \ddagger 1$ & Akkadian & MARC Language Term \\
\hline Lang & alb & $377 \ddagger 1$ & Albanian & MARC Language Term \\
\hline
\end{tabular}


Conversion Table for the Fixed Field

\begin{tabular}{|c|c|c|c|c|}
\hline \multicolumn{2}{|c|}{ Fixed Field Value } & \multicolumn{2}{|c|}{ Variable Field Value } & \multirow{2}{*}{$\begin{array}{l}\text { Notes } \\
\text { MARC Language Term }\end{array}$} \\
\hline Lang & ale & $377 \ddagger 1$ & Aleut & \\
\hline Lang & alg & $377 \ddagger 1$ & Algonquian (Other) & MARC Language Term \\
\hline Lang & alt & $377 \ddagger 1$ & Altai & MARC Language Term \\
\hline Lang & amh & $377 \ddagger 1$ & Amharic & MARC Language Term \\
\hline Lang & ang & $377 \ddagger 1$ & English, Old (ca. 450-1100) & MARC Language Term \\
\hline Lang & anp & $377 \ddagger 1$ & Angika & MARC Language Term \\
\hline Lang & ара & $377 \ddagger 1$ & Apache languages & MARC Language Term \\
\hline Lang & ara & $377 \ddagger 1$ & Arabic & MARC Language Term \\
\hline Lang & arc & $377 \ddagger 1$ & Aramaic & MARC Language Term \\
\hline Lang & $\arg$ & $377 \ddagger 1$ & Aragonese & MARC Language Term \\
\hline Lang & arm & $377 \ddagger 1$ & Armenian & MARC Language Term \\
\hline Lang & arn & $377 \ddagger 1$ & Mapuche & MARC Language Term \\
\hline Lang & arp & $377 \ddagger 1$ & Arapaho & MARC Language Term \\
\hline Lang & art & $377 \ddagger 1$ & Artificial (Other) & MARC Language Term \\
\hline Lang & arw & $377 \ddagger 1$ & Arawak & MARC Language Term \\
\hline Lang & asm & $377 \ddagger 1$ & Assamese & MARC Language Term \\
\hline Lang & ast & $377 \ddagger 1$ & Bable & MARC Language Term \\
\hline Lang & ath & $377 \ddagger 1$ & Athapascan (Other) & MARC Language Term \\
\hline Lang & aus & $377 \ddagger 1$ & Australian languages & MARC Language Term \\
\hline Lang & ava & $377 \ddagger 1$ & Avaric & MARC Language Term \\
\hline Lang & ave & $377 \ddagger 1$ & Avestan & MARC Language Term \\
\hline Lang & awa & $377 \ddagger 1$ & Awadhi & MARC Language Term \\
\hline Lang & aym & $377 \ddagger 1$ & Aymara & MARC Language Term \\
\hline Lang & aze & $377 \ddagger 1$ & Azerbaijani & MARC Language Term \\
\hline Lang & bad & $377 \ddagger 1$ & Banda languages & MARC Language Term \\
\hline Lang & bai & $377 \ddagger 1$ & Bamileke languages & MARC Language Term \\
\hline Lang & bak & $377 \ddagger 1$ & Bashkir & MARC Language Term \\
\hline Lang & bal & $377 \ddagger 1$ & Baluchi & MARC Language Term \\
\hline Lang & bam & $377 \ddagger 1$ & Bambara & MARC Language Term \\
\hline Lang & ban & $377 \ddagger 1$ & Balinese & MARC Language Term \\
\hline Lang & baq & $377 \ddagger 1$ & Basque & MARC Language Term \\
\hline Lang & bas & $377 \ddagger 1$ & Basa & MARC Language Term \\
\hline Lang & bat & $377 \ddagger 1$ & Baltic (Other) & MARC Language Term \\
\hline Lang & bej & $377 \ddagger 1$ & Beja & MARC Language Term \\
\hline Lang & bel & $377 \ddagger 1$ & Belarusian & MARC Language Term \\
\hline Lang & bem & $377 \ddagger 1$ & Bemba & MARC Language Term \\
\hline Lang & ben & $377 \ddagger 1$ & Bengali & MARC Language Term \\
\hline Lang & ber & $377 \ddagger 1$ & Berber (Other) & MARC Language Term \\
\hline Lang & bho & $377 \ddagger 1$ & Bhojpuri & MARC Language Term \\
\hline Lang & bih & $377 \ddagger 1$ & Bihari (Other) & MARC Language Term \\
\hline Lang & bik & $377 \ddagger 1$ & Bikol & MARC Language Term \\
\hline Lang & bin & $377 \ddagger 1$ & Edo & MARC Language Term \\
\hline Lang & bis & $377 \ddagger 1$ & Bislama & MARC Language Term \\
\hline Lang & bla & $377 \ddagger 1$ & Siksika & MARC Language Term \\
\hline Lang & bnt & $377 \ddagger 1$ & Bantu (Other) & MARC Language Term \\
\hline Lang & bos & $377 \ddagger 1$ & Bosnian & MARC Language Term \\
\hline Lang & bra & $377 \ddagger 1$ & Braj & MARC Language Term \\
\hline Lang & bre & $377 \ddagger 1$ & Breton & MARC Language Term \\
\hline
\end{tabular}


Conversion Table for the Fixed Field

\begin{tabular}{|c|c|c|c|c|}
\hline \multicolumn{2}{|c|}{ Fixed Field Value } & \multicolumn{2}{|c|}{ Variable Field Value } & \multirow{2}{*}{$\begin{array}{l}\text { Notes } \\
\text { MARC Language Term }\end{array}$} \\
\hline Lang & btk & $377 \ddagger 1$ & Batak & \\
\hline Lang & bua & $377 \ddagger 1$ & Buriat & MARC Language Term \\
\hline Lang & bug & $377 \ddagger 1$ & Bugis & MARC Language Term \\
\hline Lang & bul & $377 \ddagger 1$ & Bulgarian & MARC Language Term \\
\hline Lang & bur & $377 \ddagger 1$ & Burmese & MARC Language Term \\
\hline Lang & byn & $377 \ddagger 1$ & Bilin & MARC Language Term \\
\hline Lang & cad & $377 \ddagger 1$ & Caddo & MARC Language Term \\
\hline Lang & cai & $377 \ddagger 1$ & Central American Indian (Other) & MARC Language Term \\
\hline Lang & -cam & $377 \ddagger 1$ & Khmer & MARC Language Term \\
\hline Lang & car & $377 \ddagger 1$ & Carib & MARC Language Term \\
\hline Lang & cat & $377 \ddagger 1$ & Catalan & MARC Language Term \\
\hline Lang & cau & $377 \ddagger 1$ & Caucasian (Other) & MARC Language Term \\
\hline Lang & ceb & $377 \ddagger 1$ & Cebuano & MARC Language Term \\
\hline Lang & cel & $377 \ddagger 1$ & Celtic (Other) & MARC Language Term \\
\hline Lang & cha & $377 \ddagger 1$ & Chamorro & MARC Language Term \\
\hline Lang & chb & $377 \ddagger 1$ & Chibcha & MARC Language Term \\
\hline Lang & che & $377 \ddagger 1$ & Chechen & MARC Language Term \\
\hline Lang & chg & $377 \ddagger 1$ & Chagatai & MARC Language Term \\
\hline Lang & chi & $377 \ddagger 1$ & Chinese & MARC Language Term \\
\hline Lang & chk & $377 \ddagger 1$ & Chuukese & MARC Language Term \\
\hline Lang & chm & $377 \ddagger 1$ & Mari & MARC Language Term \\
\hline Lang & chn & $377 \ddagger 1$ & Chinook jargon & MARC Language Term \\
\hline Lang & cho & $377 \ddagger 1$ & Choctaw & MARC Language Term \\
\hline Lang & chp & $377 \ddagger 1$ & Chipewyan & MARC Language Term \\
\hline Lang & $\operatorname{chr}$ & $377 \ddagger 1$ & Cherokee & MARC Language Term \\
\hline Lang & chu & $377 \ddagger 1$ & Church Slavic & MARC Language Term \\
\hline Lang & chv & $377 \ddagger 1$ & Chuvash & MARC Language Term \\
\hline Lang & chy & $377 \ddagger 1$ & Cheyenne & MARC Language Term \\
\hline Lang & $\mathrm{cmc}$ & $377 \ddagger 1$ & Chamic languages & MARC Language Term \\
\hline Lang & cop & $377 \ddagger 1$ & Coptic & MARC Language Term \\
\hline Lang & cor & $377 \ddagger 1$ & Cornish & MARC Language Term \\
\hline Lang & $\cos$ & $377 \ddagger 1$ & Corsican & MARC Language Term \\
\hline Lang & cpe & $377 \ddagger 1$ & Creoles and Pidgins, English-based (Other) & MARC Language Term \\
\hline Lang & $\mathrm{cpf}$ & $377 \ddagger 1$ & Creoles and Pidgins, French-based (Other) & MARC Language Term \\
\hline Lang & cpp & $377 \ddagger 1$ & Creoles and Pidgins, Portuguese-based & MARC Language Term \\
\hline Lang & cre & $377 \ddagger 1$ & Cree & MARC Language Term \\
\hline Lang & crh & $377 \ddagger 1$ & Crimean Tatar & MARC Language Term \\
\hline Lang & crp & $377 \ddagger 1$ & Creoles and Pidgins (Other) & MARC Language Term \\
\hline Lang & $c s b$ & $377 \ddagger 1$ & Kashubian & MARC Language Term \\
\hline Lang & cus & $377 \ddagger 1$ & Cushitic (Other) & MARC Language Term \\
\hline Lang & cze & $377 \ddagger 1$ & Czech & MARC Language Term \\
\hline Lang & dak & $377 \ddagger 1$ & Dakota & MARC Language Term \\
\hline Lang & dan & $377 \ddagger 1$ & Danish & MARC Language Term \\
\hline Lang & dar & $377 \ddagger 1$ & Dargwa & MARC Language Term \\
\hline Lang & day & $377 \ddagger 1$ & Dayak & MARC Language Term \\
\hline Lang & del & $377 \ddagger 1$ & Delaware & MARC Language Term \\
\hline Lang & den & $377 \ddagger 1$ & Slavey & MARC Language Term \\
\hline Lang & dgr & $377 \ddagger 1$ & Dogrib & MARC Language Term \\
\hline
\end{tabular}


Conversion Table for the Fixed Field

\begin{tabular}{|c|c|c|c|c|}
\hline \multicolumn{2}{|c|}{ Fixed Field Value } & \multicolumn{2}{|c|}{ Variable Field Value } & \multirow{2}{*}{$\begin{array}{l}\text { Notes } \\
\text { MARC Language Term }\end{array}$} \\
\hline Lang & $\operatorname{din}$ & $377 \ddagger 1$ & Dinka & \\
\hline Lang & div & $377 \ddagger 1$ & Divehi & MARC Language Term \\
\hline Lang & doi & $377 \ddagger 1$ & Dogri & MARC Language Term \\
\hline Lang & dra & $377 \ddagger 1$ & Dravidian (Other) & MARC Language Term \\
\hline Lang & $\mathrm{dsb}$ & $377 \ddagger 1$ & Lower Sorbian & MARC Language Term \\
\hline Lang & dua & $377 \ddagger 1$ & Duala & MARC Language Term \\
\hline Lang & dum & $377 \ddagger 1$ & Dutch, Middle (ca. 1050-1350) & MARC Language Term \\
\hline Lang & dut & $377 \ddagger 1$ & Dutch & MARC Language Term \\
\hline Lang & dyu & $377 \ddagger 1$ & Dyula & MARC Language Term \\
\hline Lang & dzo & $377 \ddagger 1$ & Dzongkha & MARC Language Term \\
\hline Lang & efi & $377 \ddagger 1$ & Efik & MARC Language Term \\
\hline Lang & egy & $377 \ddagger 1$ & Egyptian & MARC Language Term \\
\hline Lang & eka & $377 \ddagger 1$ & Ekajuk & MARC Language Term \\
\hline Lang & elx & $377 \ddagger 1$ & Elamite & MARC Language Term \\
\hline Lang & eng & $377 \ddagger 1$ & English & MARC Language Term \\
\hline Lang & enm & $377 \ddagger 1$ & English, Middle (1100-1500) & MARC Language Term \\
\hline Lang & epo & $377 \ddagger 1$ & Esperanto & MARC Language Term \\
\hline Lang & -esk & $377 \ddagger 1$ & Eskimo languages & MARC Language Term \\
\hline Lang & -esp & $377 \ddagger 1$ & Esperanto & MARC Language Term \\
\hline Lang & est & $377 \ddagger 1$ & Estonian & MARC Language Term \\
\hline Lang & -eth & $377 \ddagger 1$ & Ethiopic & MARC Language Term \\
\hline Lang & ewe & $377 \ddagger 1$ & Ewe & MARC Language Term \\
\hline Lang & ewo & $377 \ddagger 1$ & Ewondo & MARC Language Term \\
\hline Lang & fan & $377 \ddagger 1$ & Fang & MARC Language Term \\
\hline Lang & fao & $377 \ddagger 1$ & Faroese & MARC Language Term \\
\hline Lang & -far & $377 \ddagger 1$ & Faroese & MARC Language Term \\
\hline Lang & fat & $377 \ddagger 1$ & Fanti & MARC Language Term \\
\hline Lang & fij & $377 \ddagger 1$ & Fijian & MARC Language Term \\
\hline Lang & fil & $377 \ddagger 1$ & Filipino & MARC Language Term \\
\hline Lang & fin & $377 \ddagger 1$ & Finnish & MARC Language Term \\
\hline Lang & fiu & $377 \ddagger 1$ & Finno-Ugrian (Other) & MARC Language Term \\
\hline Lang & fon & $377 \ddagger 1$ & Fon & MARC Language Term \\
\hline Lang & fre & $377 \ddagger 1$ & French & MARC Language Term \\
\hline Lang & -fri & $377 \ddagger 1$ & Frisian & MARC Language Term \\
\hline Lang & frm & $377 \ddagger 1$ & French, Middle (ca. 1300-1600) & MARC Language Term \\
\hline Lang & fro & $377 \ddagger 1$ & French, Old (ca. 842-1300) & MARC Language Term \\
\hline Lang & frr & $377 \ddagger 1$ & North Frisian & MARC Language Term \\
\hline Lang & frs & $377 \ddagger 1$ & East Frisian & MARC Language Term \\
\hline Lang & fry & $377 \ddagger 1$ & Frisian & MARC Language Term \\
\hline Lang & ful & $377 \ddagger 1$ & Fula & MARC Language Term \\
\hline Lang & fur & $377 \ddagger 1$ & Friulian & MARC Language Term \\
\hline Lang & gaa & $377 \ddagger 1$ & Gã & MARC Language Term \\
\hline Lang & -gae & $377 \ddagger 1$ & Scottish Gaelix & MARC Language Term \\
\hline Lang & -gag & $377 \ddagger 1$ & Galician & MARC Language Term \\
\hline Lang & -gal & $377 \ddagger 1$ & Oromo & MARC Language Term \\
\hline Lang & gay & $377 \ddagger 1$ & Gayo & MARC Language Term \\
\hline Lang & gba & $377 \ddagger 1$ & Gbaya & MARC Language Term \\
\hline Lang & gem & $377 \ddagger 1$ & Germanic (Other) & MARC Language Term \\
\hline
\end{tabular}


Conversion Table for the Fixed Field

\begin{tabular}{|c|c|c|c|c|}
\hline \multicolumn{2}{|c|}{ Fixed Field Value } & \multicolumn{2}{|c|}{ Variable Field Value } & \multirow{2}{*}{$\begin{array}{l}\text { Notes } \\
\text { MARC Language Term }\end{array}$} \\
\hline Lang & geo & $377 \ddagger 1$ & Georgian & \\
\hline Lang & ger & $377 \ddagger 1$ & German & MARC Language Term \\
\hline Lang & gez & $377 \ddagger 1$ & Ethiopic & MARC Language Term \\
\hline Lang & gil & $377 \ddagger 1$ & Gilbertese & MARC Language Term \\
\hline Lang & gla & $377 \ddagger 1$ & Scottish Gaelic & MARC Language Term \\
\hline Lang & gle & $377 \ddagger 1$ & Irish & MARC Language Term \\
\hline Lang & glg & $377 \ddagger 1$ & Galician & MARC Language Term \\
\hline Lang & glv & $377 \ddagger 1$ & Manx & MARC Language Term \\
\hline Lang & gmh & $377 \ddagger 1$ & German, Middle High (ca. 1050-1500) & MARC Language Term \\
\hline Lang & goh & $377 \ddagger 1$ & German, Old High (ca. 750-1050) & MARC Language Term \\
\hline Lang & gon & $377 \ddagger 1$ & Gondi & MARC Language Term \\
\hline Lang & gor & $377 \ddagger 1$ & Gorontalo & MARC Language Term \\
\hline Lang & got & $377 \ddagger 1$ & Gothic & MARC Language Term \\
\hline Lang & grb & $377 \ddagger 1$ & Grebo & MARC Language Term \\
\hline Lang & grc & $377 \ddagger 1$ & Greek, Ancient (to 1453) & MARC Language Term \\
\hline Lang & gre & $377 \ddagger 1$ & Greek, Modern (1453-) & MARC Language Term \\
\hline Lang & grn & $377 \ddagger 1$ & Guarani & MARC Language Term \\
\hline Lang & gsw & $377 \ddagger 1$ & Swiss German & MARC Language Term \\
\hline Lang & -gua & $377 \ddagger 1$ & Guarani & MARC Language Term \\
\hline Lang & guj & $377 \ddagger 1$ & Gujarati & MARC Language Term \\
\hline Lang & gwi & $377 \ddagger 1$ & Gwich'in & MARC Language Term \\
\hline Lang & hai & $377 \ddagger 1$ & Haida & MARC Language Term \\
\hline Lang & hat & $377 \ddagger 1$ & Haitian French Creole & MARC Language Term \\
\hline Lang & hau & $377 \ddagger 1$ & Hausa & MARC Language Term \\
\hline Lang & haw & $377 \ddagger 1$ & Hawaiian & MARC Language Term \\
\hline Lang & heb & $377 \ddagger 1$ & Hebrew & MARC Language Term \\
\hline Lang & her & $377 \ddagger 1$ & Herero & MARC Language Term \\
\hline Lang & hil & $377 \ddagger 1$ & Hiligaynon & MARC Language Term \\
\hline Lang & him & $377 \ddagger 1$ & Western Pahari languages & MARC Language Term \\
\hline Lang & hin & $377 \ddagger 1$ & Hindi & MARC Language Term \\
\hline Lang & hit & $377 \ddagger 1$ & Hittite & MARC Language Term \\
\hline Lang & $\mathrm{hmn}$ & $377 \ddagger 1$ & Hmong & MARC Language Term \\
\hline Lang & hmo & $377 \ddagger 1$ & Hiri Motu & MARC Language Term \\
\hline Lang & hrv & $377 \ddagger 1$ & Croatian & MARC Language Term \\
\hline Lang & hsb & $377 \ddagger 1$ & Upper Sorbian & MARC Language Term \\
\hline Lang & hun & $377 \ddagger 1$ & Hungarian & MARC Language Term \\
\hline Lang & hup & $377 \ddagger 1$ & Hupa & MARC Language Term \\
\hline Lang & iba & $377 \ddagger 1$ & Iban & MARC Language Term \\
\hline Lang & ibo & $377 \ddagger 1$ & Igbo & MARC Language Term \\
\hline Lang & ice & $377 \neq 1$ & Icelandic & MARC Language Term \\
\hline Lang & ido & $377 \ddagger 1$ & Ido & MARC Language Term \\
\hline Lang & iii & $377 \ddagger 1$ & Sichuan Yi & MARC Language Term \\
\hline Lang & ijo & $377 \ddagger 1$ & ljo & MARC Language Term \\
\hline Lang & iku & $377 \ddagger 1$ & Inuktitut & MARC Language Term \\
\hline Lang & ile & $377 \ddagger 1$ & Interlingue & MARC Language Term \\
\hline Lang & ilo & $377 \ddagger 1$ & lloko & MARC Language Term \\
\hline Lang & ina & $377 \ddagger 1$ & Interlingua (International Auxiliary Language & MARC Language Term \\
\hline Lang & inc & $377 \ddagger 1$ & Indic (Other) & MARC Language Term \\
\hline
\end{tabular}


Conversion Table for the Fixed Field

\begin{tabular}{|c|c|c|c|c|}
\hline \multicolumn{2}{|c|}{ Fixed Field Value } & \multicolumn{2}{|c|}{ Variable Field Value } & \multirow{2}{*}{$\begin{array}{l}\text { Notes } \\
\text { MARC Language Term }\end{array}$} \\
\hline Lang & ind & $377 \ddagger 1$ & Indonesian & \\
\hline Lang & ine & $377 \ddagger 1$ & Indo-European (Other) & MARC Language Term \\
\hline Lang & inh & $377 \ddagger 1$ & Ingush & MARC Language Term \\
\hline Lang & -int & $377 \ddagger 1$ & Interlingua (International Auxiliary Language & MARC Language Term \\
\hline Lang & ipk & $377 \ddagger 1$ & Inupiaq & MARC Language Term \\
\hline Lang & ira & $377 \ddagger 1$ & Iranian (Other) & MARC Language Term \\
\hline Lang & -iri & $377 \ddagger 1$ & Irish & MARC Language Term \\
\hline Lang & iro & $377 \ddagger 1$ & Iroquoian (Other) & MARC Language Term \\
\hline Lang & ita & $377 \ddagger 1$ & Italian & MARC Language Term \\
\hline Lang & jav & $377 \ddagger 1$ & Javanese & MARC Language Term \\
\hline Lang & jbo & $377 \ddagger 1$ & Lojban (Artificial language) & MARC Language Term \\
\hline Lang & jpn & $377 \ddagger 1$ & Japanese & MARC Language Term \\
\hline Lang & jpr & $377 \ddagger 1$ & Judeo-Persian & MARC Language Term \\
\hline Lang & jrb & $377 \ddagger 1$ & Judeo-Arabic & MARC Language Term \\
\hline Lang & kaa & $377 \ddagger 1$ & Kara-Kalpak & MARC Language Term \\
\hline Lang & $\mathrm{kab}$ & $377 \ddagger 1$ & Kabyle & MARC Language Term \\
\hline Lang & kac & $377 \ddagger 1$ & Kachin & MARC Language Term \\
\hline Lang & kal & $377 \ddagger 1$ & Kalâtdlisut & MARC Language Term \\
\hline Lang & kam & $377 \ddagger 1$ & Kamba & MARC Language Term \\
\hline Lang & kan & $377 \ddagger 1$ & Kannada & MARC Language Term \\
\hline Lang & kar & $377 \ddagger 1$ & Karen languages & MARC Language Term \\
\hline Lang & kas & $377 \ddagger 1$ & Kashmiri & MARC Language Term \\
\hline Lang & kau & $377 \ddagger 1$ & Kanuri & MARC Language Term \\
\hline Lang & kaw & $377 \ddagger 1$ & Kawi & MARC Language Term \\
\hline Lang & kaz & $377 \ddagger 1$ & Kazakh & MARC Language Term \\
\hline Lang & $\mathrm{kbd}$ & $377 \ddagger 1$ & Kabardian & MARC Language Term \\
\hline Lang & kha & $377 \ddagger 1$ & Khasi & MARC Language Term \\
\hline Lang & khi & $377 \ddagger 1$ & Khoisan (Other) & MARC Language Term \\
\hline Lang & $\mathrm{khm}$ & $377 \ddagger 1$ & Khmer & MARC Language Term \\
\hline Lang & kho & $377 \ddagger 1$ & Khotanese & MARC Language Term \\
\hline Lang & kik & $377 \ddagger 1$ & Kikuyu & MARC Language Term \\
\hline Lang & kin & $377 \ddagger 1$ & Kinyarwanda & MARC Language Term \\
\hline Lang & kir & $377 \ddagger 1$ & Kyrgyz & MARC Language Term \\
\hline Lang & $\mathrm{kmb}$ & $377 \ddagger 1$ & Kimbundu & MARC Language Term \\
\hline Lang & kok & $377 \ddagger 1$ & Konkani & MARC Language Term \\
\hline Lang & kom & $377 \ddagger 1$ & Komi & MARC Language Term \\
\hline Lang & kon & $377 \ddagger 1$ & Kongo & MARC Language Term \\
\hline Lang & kor & $377 \ddagger 1$ & Korean & MARC Language Term \\
\hline Lang & kos & $377 \ddagger 1$ & Kosraean & MARC Language Term \\
\hline Lang & kpe & $377 \ddagger 1$ & Kpelle & MARC Language Term \\
\hline Lang & $\mathrm{krc}$ & $377 \ddagger 1$ & Karachay-Balkar & MARC Language Term \\
\hline Lang & $\mathrm{krl}$ & $377 \ddagger 1$ & Karelian & MARC Language Term \\
\hline Lang & kro & $377 \ddagger 1$ & Kru (Other) & MARC Language Term \\
\hline Lang & kru & $377 \ddagger 1$ & Kurukh & MARC Language Term \\
\hline Lang & kua & $377 \ddagger 1$ & Kuanyama & MARC Language Term \\
\hline Lang & kum & $377 \ddagger 1$ & Kumyk & MARC Language Term \\
\hline Lang & kur & $377 \ddagger 1$ & Kurdish & MARC Language Term \\
\hline Lang & -kus & $377 \ddagger 1$ & Kusaie & MARC Language Term \\
\hline
\end{tabular}


Conversion Table for the Fixed Field

\begin{tabular}{|c|c|c|c|c|}
\hline \multicolumn{2}{|c|}{ Fixed Field Value } & \multicolumn{2}{|c|}{ Variable Field Value } & \multirow{2}{*}{$\begin{array}{l}\text { Notes } \\
\text { MARC Language Term }\end{array}$} \\
\hline Lang & kut & $377 \ddagger 1$ & Kootenai & \\
\hline Lang & lad & $377 \ddagger 1$ & Ladino & MARC Language Term \\
\hline Lang & lah & $377 \ddagger 1$ & Lahnd? & MARC Language Term \\
\hline Lang & lam & $377 \ddagger 1$ & Lamba (Zambia and Congo) & MARC Language Term \\
\hline Lang & $-\operatorname{lan}$ & $377 \ddagger 1$ & Occitan (post 1500) & MARC Language Term \\
\hline Lang & lao & $377 \ddagger 1$ & Lao & MARC Language Term \\
\hline Lang & -lap & $377 \ddagger 1$ & Sami & MARC Language Term \\
\hline Lang & lat & $377 \ddagger 1$ & Latin & MARC Language Term \\
\hline Lang & lav & $377 \ddagger 1$ & Latvian & MARC Language Term \\
\hline Lang & lez & $377 \ddagger 1$ & Lezgian & MARC Language Term \\
\hline Lang & $\lim$ & $377 \ddagger 1$ & Limburgish & MARC Language Term \\
\hline Lang & $\operatorname{lin}$ & $377 \ddagger 1$ & Lingala & MARC Language Term \\
\hline Lang & lit & $377 \ddagger 1$ & Lithuanian & MARC Language Term \\
\hline Lang & lol & $377 \ddagger 1$ & Mongo-Nkundu & MARC Language Term \\
\hline Lang & loz & $377 \ddagger 1$ & Lozi & MARC Language Term \\
\hline Lang & Itz & $377 \ddagger 1$ & Luxembourgish & MARC Language Term \\
\hline Lang & lua & $377 \ddagger 1$ & Luba-Lulua & MARC Language Term \\
\hline Lang & lub & $377 \ddagger 1$ & Luba-Katanga & MARC Language Term \\
\hline Lang & lug & $377 \ddagger 1$ & Ganda & MARC Language Term \\
\hline Lang & lui & $377 \ddagger 1$ & Luiseño & MARC Language Term \\
\hline Lang & lun & $377 \ddagger 1$ & Lunda & MARC Language Term \\
\hline Lang & luo & $377 \ddagger 1$ & Luo (Kenya and Tanzania) & MARC Language Term \\
\hline Lang & lus & $377 \ddagger 1$ & Lushai & MARC Language Term \\
\hline Lang & mac & $377 \ddagger 1$ & Macedonian & MARC Language Term \\
\hline Lang & mad & $377 \ddagger 1$ & Madurese & MARC Language Term \\
\hline Lang & mag & $377 \ddagger 1$ & Magahi & MARC Language Term \\
\hline Lang & mah & $377 \ddagger 1$ & Marshallese & MARC Language Term \\
\hline Lang & mai & $377 \ddagger 1$ & Maithili & MARC Language Term \\
\hline Lang & mak & $377 \ddagger 1$ & Makasar & MARC Language Term \\
\hline Lang & mal & $377 \ddagger 1$ & Malayalam & MARC Language Term \\
\hline Lang & man & $377 \ddagger 1$ & Mandingo & MARC Language Term \\
\hline Lang & mao & $377 \ddagger 1$ & Maori & MARC Language Term \\
\hline Lang & map & $377 \ddagger 1$ & Austronesian (Other) & MARC Language Term \\
\hline Lang & mar & $377 \ddagger 1$ & Marathi & MARC Language Term \\
\hline Lang & mas & $377 \ddagger 1$ & Maasai & MARC Language Term \\
\hline Lang & $-\max$ & $377 \ddagger 1$ & Manx & MARC Language Term \\
\hline Lang & may & $377 \ddagger 1$ & Malay & MARC Language Term \\
\hline Lang & mdf & $377 \ddagger 1$ & Moksha & MARC Language Term \\
\hline Lang & $\mathrm{mdr}$ & $377 \ddagger 1$ & Mandar & MARC Language Term \\
\hline Lang & men & $377 \ddagger 1$ & Mende & MARC Language Term \\
\hline Lang & mga & $377 \ddagger 1$ & Irish, Middle (ca. 1100-1550) & MARC Language Term \\
\hline Lang & mic & $377 \ddagger 1$ & Micmac & MARC Language Term \\
\hline Lang & $\min$ & $377 \ddagger 1$ & Minangkabau & MARC Language Term \\
\hline Lang & mis & $377 \ddagger 1$ & Miscellaneous languages & MARC Language Term \\
\hline Lang & $\mathrm{mkh}$ & $377 \ddagger 1$ & Mon-Khmer (Other) & MARC Language Term \\
\hline Lang & - mla & $377 \ddagger 1$ & Malagasy & MARC Language Term \\
\hline Lang & $\mathrm{mlg}$ & $377 \ddagger 1$ & Malagasy & MARC Language Term \\
\hline Lang & $\mathrm{mlt}$ & $377 \ddagger 1$ & Maltese & MARC Language Term \\
\hline
\end{tabular}


Conversion Table for the Fixed Field

\begin{tabular}{|c|c|c|c|c|}
\hline \multicolumn{2}{|c|}{ Fixed Field Value } & \multicolumn{2}{|c|}{ Variable Field Value } & \multirow{2}{*}{$\begin{array}{l}\text { Notes } \\
\text { MARC Language Term }\end{array}$} \\
\hline Lang & mnc & $377 \ddagger 1$ & Manchu & \\
\hline Lang & $\mathrm{mni}$ & $377 \ddagger 1$ & Manipuri & MARC Language Term \\
\hline Lang & mno & $377 \ddagger 1$ & Manobo languages & MARC Language Term \\
\hline Lang & moh & $377 \ddagger 1$ & Mohawk & MARC Language Term \\
\hline Lang & $-\mathrm{mol}$ & $377 \ddagger 1$ & Moldavian & MARC Language Term \\
\hline Lang & mon & $377 \ddagger 1$ & Mongolian & MARC Language Term \\
\hline Lang & mos & $377 \ddagger 1$ & Mooré & MARC Language Term \\
\hline Lang & mul & $377 \ddagger 1$ & Multiple languages & MARC Language Term \\
\hline Lang & mun & $377 \ddagger 1$ & Munda (Other) & MARC Language Term \\
\hline Lang & mus & $377 \ddagger 1$ & Creek & MARC Language Term \\
\hline Lang & $\mathrm{mwl}$ & $377 \ddagger 1$ & Mirandese & MARC Language Term \\
\hline Lang & mwr & $377 \ddagger 1$ & Marwari & MARC Language Term \\
\hline Lang & myn & $377 \ddagger 1$ & Mayan languages & MARC Language Term \\
\hline Lang & myv & $377 \ddagger 1$ & Erzya & MARC Language Term \\
\hline Lang & nah & $377 \ddagger 1$ & Nahuatl & MARC Language Term \\
\hline Lang & nai & $377 \ddagger 1$ & North American Indian (Other) & MARC Language Term \\
\hline Lang & nap & $377 \ddagger 1$ & Neapolitan Italian & MARC Language Term \\
\hline Lang & nau & $377 \ddagger 1$ & Nauru & MARC Language Term \\
\hline Lang & nav & $377 \ddagger 1$ & Navajo & MARC Language Term \\
\hline Lang & $\mathrm{nbl}$ & $377 \ddagger 1$ & Ndebele (South Africa) & MARC Language Term \\
\hline Lang & nde & $377 \ddagger 1$ & Ndebele (Zimbabwe) & MARC Language Term \\
\hline Lang & ndo & $377 \ddagger 1$ & Ndonga & MARC Language Term \\
\hline Lang & nds & $377 \ddagger 1$ & Low German & MARC Language Term \\
\hline Lang & nep & $377 \ddagger 1$ & Nepali & MARC Language Term \\
\hline Lang & new & $377 \ddagger 1$ & Newari & MARC Language Term \\
\hline Lang & nia & $377 \ddagger 1$ & Nias & MARC Language Term \\
\hline Lang & nic & $377 \ddagger 1$ & Niger-Kordofanian (Other) & MARC Language Term \\
\hline Lang & niu & $377 \ddagger 1$ & Niuean & MARC Language Term \\
\hline Lang & nno & $377 \ddagger 1$ & Norwegian (Nynorsk) & MARC Language Term \\
\hline Lang & nob & $377 \ddagger 1$ & Norwegian (Bokmål) & MARC Language Term \\
\hline Lang & nog & $377 \ddagger 1$ & Nogai & MARC Language Term \\
\hline Lang & non & $377 \ddagger 1$ & Old Norse & MARC Language Term \\
\hline Lang & nor & $377 \ddagger 1$ & Norwegian & MARC Language Term \\
\hline Lang & nqo & $377 \ddagger 1$ & N'Ko & MARC Language Term \\
\hline Lang & nso & $377 \ddagger 1$ & Northern Sotho & MARC Language Term \\
\hline Lang & nub & $377 \ddagger 1$ & Nubian languages & MARC Language Term \\
\hline Lang & nwc & $377 \ddagger 1$ & Newari, Old & MARC Language Term \\
\hline Lang & nya & $377 \ddagger 1$ & Nyanja & MARC Language Term \\
\hline Lang & nym & $377 \ddagger 1$ & Nyamwezi & MARC Language Term \\
\hline Lang & nyn & $377 \neq 1$ & Nyankole & MARC Language Term \\
\hline Lang & nyo & $377 \ddagger 1$ & Nyoro & MARC Language Term \\
\hline Lang & nzi & $377 \neq 1$ & Nzima & MARC Language Term \\
\hline Lang & oci & $377 \ddagger 1$ & Occitan (post 1500) & MARC Language Term \\
\hline Lang & oji & $377 \ddagger 1$ & Ojibwa & MARC Language Term \\
\hline Lang & ori & $377 \ddagger 1$ & Oriya & MARC Language Term \\
\hline Lang & orm & $377 \ddagger 1$ & Oromo & MARC Language Term \\
\hline Lang & osa & $377 \ddagger 1$ & Osage & MARC Language Term \\
\hline Lang & oss & $377 \ddagger 1$ & Ossetic & MARC Language Term \\
\hline
\end{tabular}


Conversion Table for the Fixed Field

\begin{tabular}{|c|c|c|c|c|}
\hline \multicolumn{2}{|c|}{ Fixed Field Value } & \multicolumn{2}{|c|}{ Variable Field Value } & \multirow{2}{*}{$\begin{array}{l}\text { Notes } \\
\text { MARC Language Term }\end{array}$} \\
\hline Lang & ota & $377 \ddagger 1$ & Turkish, Ottoman & \\
\hline Lang & oto & $377 \ddagger 1$ & Otomian languages & MARC Language Term \\
\hline Lang & paa & $377 \ddagger 1$ & Papuan (Other) & MARC Language Term \\
\hline Lang & pag & $377 \ddagger 1$ & Pangasinan & MARC Language Term \\
\hline Lang & pal & $377 \ddagger 1$ & Pahlavi & MARC Language Term \\
\hline Lang & pam & $377 \ddagger 1$ & Pampanga & MARC Language Term \\
\hline Lang & pan & $377 \ddagger 1$ & Panjabi & MARC Language Term \\
\hline Lang & pap & $377 \ddagger 1$ & Papiamento & MARC Language Term \\
\hline Lang & pau & $377 \ddagger 1$ & Palauan & MARC Language Term \\
\hline Lang & peo & $377 \ddagger 1$ & Old Persian (ca. $600-400$ B.C.) & MARC Language Term \\
\hline Lang & per & $377 \ddagger 1$ & Persian & MARC Language Term \\
\hline Lang & phi & $377 \ddagger 1$ & Philippine (Other) & MARC Language Term \\
\hline Lang & phn & $377 \ddagger 1$ & Phoenician & MARC Language Term \\
\hline Lang & pli & $377 \ddagger 1$ & Pali & MARC Language Term \\
\hline Lang & pol & $377 \ddagger 1$ & Polish & MARC Language Term \\
\hline Lang & pon & $377 \ddagger 1$ & Pohnpeian & MARC Language Term \\
\hline Lang & por & $377 \ddagger 1$ & Portuguese & MARC Language Term \\
\hline Lang & pra & $377 \ddagger 1$ & Prakrit languages & MARC Language Term \\
\hline Lang & pro & $377 \ddagger 1$ & Provençal (to 1500) & MARC Language Term \\
\hline Lang & pus & $377 \ddagger 1$ & Pushto & MARC Language Term \\
\hline Lang & que & $377 \ddagger 1$ & Quechua & MARC Language Term \\
\hline Lang & raj & $377 \ddagger 1$ & Rajasthani & MARC Language Term \\
\hline Lang & rap & $377 \ddagger 1$ & Rapanui & MARC Language Term \\
\hline Lang & rar & $377 \ddagger 1$ & Rarotongan & MARC Language Term \\
\hline Lang & roa & $377 \ddagger 1$ & Romance (Other) & MARC Language Term \\
\hline Lang & roh & $377 \ddagger 1$ & Raeto-Romance & MARC Language Term \\
\hline Lang & rom & $377 \ddagger 1$ & Romani & MARC Language Term \\
\hline Lang & rum & $377 \ddagger 1$ & Romanian & MARC Language Term \\
\hline Lang & run & $377 \ddagger 1$ & Rundi & MARC Language Term \\
\hline Lang & rup & $377 \ddagger 1$ & Aromanian & MARC Language Term \\
\hline Lang & rus & $377 \ddagger 1$ & Russian & MARC Language Term \\
\hline Lang & sad & $377 \ddagger 1$ & Sandawe & MARC Language Term \\
\hline Lang & sag & $377 \ddagger 1$ & Sango (Ubangi Creole) & MARC Language Term \\
\hline Lang & sah & $377 \ddagger 1$ & Yakut & MARC Language Term \\
\hline Lang & sai & $377 \ddagger 1$ & South American Indian (Other) & MARC Language Term \\
\hline Lang & sal & $377 \ddagger 1$ & Salishan languages & MARC Language Term \\
\hline Lang & sam & $377 \ddagger 1$ & Samaritan Aramaic & MARC Language Term \\
\hline Lang & san & $377 \ddagger 1$ & Sanskrit & MARC Language Term \\
\hline Lang & -sao & $377 \ddagger 1$ & Samoan & MARC Language Term \\
\hline Lang & sas & $377 \ddagger 1$ & Sasak & MARC Language Term \\
\hline Lang & sat & $377 \ddagger 1$ & Santali & MARC Language Term \\
\hline Lang & $-\mathrm{scc}$ & $377 \ddagger 1$ & Serbian & MARC Language Term \\
\hline Lang & scn & $377 \ddagger 1$ & Sicilian Italian & MARC Language Term \\
\hline Lang & sco & $377 \ddagger 1$ & Scots & MARC Language Term \\
\hline Lang & $-\mathrm{scr}$ & $377 \ddagger 1$ & Croatian & MARC Language Term \\
\hline Lang & sel & $377 \ddagger 1$ & Selkup & MARC Language Term \\
\hline Lang & sem & $377 \ddagger 1$ & Semitic (Other) & MARC Language Term \\
\hline Lang & sga & $377 \ddagger 1$ & Irish, Old (to 1100) & MARC Language Term \\
\hline
\end{tabular}


Conversion Table for the Fixed Field

\begin{tabular}{|c|c|c|c|c|}
\hline \multicolumn{2}{|c|}{ Fixed Field Value } & \multicolumn{2}{|c|}{ Variable Field Value } & \multirow{2}{*}{$\begin{array}{l}\text { Notes } \\
\text { MARC Language Term }\end{array}$} \\
\hline Lang & sgn & $377 \ddagger 1$ & Sign languages & \\
\hline Lang & shn & $377 \ddagger 1$ & Shan & MARC Language Term \\
\hline Lang & -sho & $377 \ddagger 1$ & Shona & MARC Language Term \\
\hline Lang & sid & $377 \ddagger 1$ & Sidamo & MARC Language Term \\
\hline Lang & $\sin$ & $377 \ddagger 1$ & Sinhalese & MARC Language Term \\
\hline Lang & sio & $377 \ddagger 1$ & Siouan (Other) & MARC Language Term \\
\hline Lang & sit & $377 \ddagger 1$ & Sino-Tibetan (Other) & MARC Language Term \\
\hline Lang & sla & $377 \ddagger 1$ & Slavic (Other) & MARC Language Term \\
\hline Lang & slo & $377 \ddagger 1$ & Slovak & MARC Language Term \\
\hline Lang & slv & $377 \ddagger 1$ & Slovenian & MARC Language Term \\
\hline Lang & sma & $377 \ddagger 1$ & Southern Sami & MARC Language Term \\
\hline Lang & sme & $377 \ddagger 1$ & Northern Sami & MARC Language Term \\
\hline Lang & smi & $377 \ddagger 1$ & Sami & MARC Language Term \\
\hline Lang & smj & $377 \ddagger 1$ & Lule Sami & MARC Language Term \\
\hline Lang & smn & $377 \ddagger 1$ & Inari Sami & MARC Language Term \\
\hline Lang & smo & $377 \ddagger 1$ & Samoan & MARC Language Term \\
\hline Lang & sms & $377 \ddagger 1$ & Skolt Sami & MARC Language Term \\
\hline Lang & sna & $377 \ddagger 1$ & Shona & MARC Language Term \\
\hline Lang & snd & $377 \ddagger 1$ & Sindhi & MARC Language Term \\
\hline Lang & - snh & $377 \ddagger 1$ & Sinhalese & MARC Language Term \\
\hline Lang & snk & $377 \ddagger 1$ & Soninke & MARC Language Term \\
\hline Lang & sog & $377 \ddagger 1$ & Sogdian & MARC Language Term \\
\hline Lang & som & $377 \ddagger 1$ & Somali & MARC Language Term \\
\hline Lang & son & $377 \ddagger 1$ & Songhai & MARC Language Term \\
\hline Lang & sot & $377 \ddagger 1$ & Sotho & MARC Language Term \\
\hline Lang & spa & $377 \ddagger 1$ & Spanish & MARC Language Term \\
\hline Lang & srd & $377 \ddagger 1$ & Sardinian & MARC Language Term \\
\hline Lang & srn & $377 \ddagger 1$ & Sranan & MARC Language Term \\
\hline Lang & srp & $377 \ddagger 1$ & Serbian & MARC Language Term \\
\hline Lang & srr & $377 \ddagger 1$ & Serer & MARC Language Term \\
\hline Lang & ssa & $377 \ddagger 1$ & Nilo-Saharan (Other) & MARC Language Term \\
\hline Lang & -sso & $377 \ddagger 1$ & Sotho & MARC Language Term \\
\hline Lang & ssw & $377 \ddagger 1$ & Swazi & MARC Language Term \\
\hline Lang & suk & $377 \ddagger 1$ & Sukuma & MARC Language Term \\
\hline Lang & sun & $377 \ddagger 1$ & Sundanese & MARC Language Term \\
\hline Lang & sus & $377 \ddagger 1$ & Susu & MARC Language Term \\
\hline Lang & sux & $377 \ddagger 1$ & Sumerian & MARC Language Term \\
\hline Lang & swa & $377 \ddagger 1$ & Swahili & MARC Language Term \\
\hline Lang & swe & $377 \ddagger 1$ & Swedish & MARC Language Term \\
\hline Lang & $-s w z$ & $377 \ddagger 1$ & Swazi & MARC Language Term \\
\hline Lang & syc & $377 \ddagger 1$ & Syriac & MARC Language Term \\
\hline Lang & syr & $377 \ddagger 1$ & Syriac, Modern & MARC Language Term \\
\hline Lang & -tag & $377 \ddagger 1$ & Tagalog & MARC Language Term \\
\hline Lang & tah & $377 \ddagger 1$ & Tahitian & MARC Language Term \\
\hline Lang & tai & $377 \ddagger 1$ & Tai (Other) & MARC Language Term \\
\hline Lang & -taj & $377 \ddagger 1$ & Tajik & MARC Language Term \\
\hline Lang & tam & $377 \ddagger 1$ & Tamil & MARC Language Term \\
\hline Lang & $-\operatorname{tar}$ & $377 \ddagger 1$ & Tatar & MARC Language Term \\
\hline
\end{tabular}


Conversion Table for the Fixed Field

\begin{tabular}{|c|c|c|c|c|}
\hline \multicolumn{2}{|c|}{ Fixed Field Value } & \multicolumn{2}{|c|}{ Variable Field Value } & \multirow{2}{*}{$\begin{array}{l}\text { Notes } \\
\text { MARC Language Term }\end{array}$} \\
\hline Lang & tat & $377 \ddagger 1$ & Tatar & \\
\hline Lang & tel & $377 \ddagger 1$ & Telugu & MARC Language Term \\
\hline Lang & tem & $377 \ddagger 1$ & Temne & MARC Language Term \\
\hline Lang & ter & $377 \ddagger 1$ & Terena & MARC Language Term \\
\hline Lang & tet & $377 \ddagger 1$ & Tetum & MARC Language Term \\
\hline Lang & $\operatorname{tgk}$ & $377 \ddagger 1$ & Tajik & MARC Language Term \\
\hline Lang & $\operatorname{tgl}$ & $377 \ddagger 1$ & Tagalog & MARC Language Term \\
\hline Lang & tha & $377 \ddagger 1$ & Thai & MARC Language Term \\
\hline Lang & tib & $377 \ddagger 1$ & Tibetan & MARC Language Term \\
\hline Lang & tig & $377 \ddagger 1$ & Tigré & MARC Language Term \\
\hline Lang & tir & $377 \ddagger 1$ & Tigrinya & MARC Language Term \\
\hline Lang & tiv & $377 \ddagger 1$ & Tiv & MARC Language Term \\
\hline Lang & tkl & $377 \ddagger 1$ & Tokelauan & MARC Language Term \\
\hline Lang & th & $377 \ddagger 1$ & Klingon (Artificial language) & MARC Language Term \\
\hline Lang & $\mathrm{tli}$ & $377 \ddagger 1$ & Tlingit & MARC Language Term \\
\hline Lang & $\mathrm{tmh}$ & $377 \ddagger 1$ & Tamashek & MARC Language Term \\
\hline Lang & tog & $377 \ddagger 1$ & Tonga (Nyasa) & MARC Language Term \\
\hline Lang & ton & $377 \ddagger 1$ & Tongan & MARC Language Term \\
\hline Lang & tpi & $377 \ddagger 1$ & Tok Pisin & MARC Language Term \\
\hline Lang & -tru & $377 \ddagger 1$ & Truk & MARC Language Term \\
\hline Lang & tsi & $377 \ddagger 1$ & Tsimshian & MARC Language Term \\
\hline Lang & tsn & $377 \ddagger 1$ & Tswana & MARC Language Term \\
\hline Lang & tso & $377 \ddagger 1$ & Tsonga & MARC Language Term \\
\hline Lang & $-\mathrm{tsw}$ & $377 \ddagger 1$ & Tswana & MARC Language Term \\
\hline Lang & tuk & $377 \ddagger 1$ & Turkmen & MARC Language Term \\
\hline Lang & tum & $377 \ddagger 1$ & Tumbuka & MARC Language Term \\
\hline Lang & tup & $377 \ddagger 1$ & Tupi languages & MARC Language Term \\
\hline Lang & tur & $377 \ddagger 1$ & Turkish & MARC Language Term \\
\hline Lang & tut & $377 \ddagger 1$ & Altaic (Other) & MARC Language Term \\
\hline Lang & $\mathrm{tvl}$ & $377 \ddagger 1$ & Tuvaluan & MARC Language Term \\
\hline Lang & twi & $377 \ddagger 1$ & Twi & MARC Language Term \\
\hline Lang & tyv & $377 \ddagger 1$ & Tuvinian & MARC Language Term \\
\hline Lang & udm & $377 \ddagger 1$ & Udmurt & MARC Language Term \\
\hline Lang & uga & $377 \ddagger 1$ & Ugaritic & MARC Language Term \\
\hline Lang & uig & $377 \ddagger 1$ & Uighur & MARC Language Term \\
\hline Lang & ukr & $377 \ddagger 1$ & Ukrainian & MARC Language Term \\
\hline Lang & umb & $377 \ddagger 1$ & Umbundu & MARC Language Term \\
\hline Lang & und & $377 \ddagger 1$ & Undetermined & MARC Language Term \\
\hline Lang & urd & $377 \ddagger 1$ & Urdu & MARC Language Term \\
\hline Lang & uzb & $377 \ddagger 1$ & Uzbek & MARC Language Term \\
\hline Lang & vai & $377 \ddagger 1$ & Vai & MARC Language Term \\
\hline Lang & ven & $377 \ddagger 1$ & Venda & MARC Language Term \\
\hline Lang & vie & $377 \ddagger 1$ & Vietnamese & MARC Language Term \\
\hline Lang & vol & $377 \ddagger 1$ & Volapük & MARC Language Term \\
\hline Lang & vot & $377 \ddagger 1$ & Votic & MARC Language Term \\
\hline Lang & wak & $377 \ddagger 1$ & Wakashan languages & MARC Language Term \\
\hline Lang & wal & $377 \ddagger 1$ & Wolayta & MARC Language Term \\
\hline Lang & war & $377 \ddagger 1$ & Waray & MARC Language Term \\
\hline
\end{tabular}


Conversion Table for the Fixed Field

\begin{tabular}{|c|c|c|c|c|}
\hline \multicolumn{2}{|c|}{ Fixed Field Value } & \multicolumn{2}{|c|}{ Variable Field Value } & \multirow{2}{*}{$\begin{array}{l}\text { Notes } \\
\text { MARC Language Term }\end{array}$} \\
\hline Lang & was & $377 \ddagger 1$ & Washoe & \\
\hline Lang & wel & $377 \ddagger 1$ & Welsh & MARC Language Term \\
\hline Lang & wen & $377 \ddagger 1$ & Sorbian (Other) & MARC Language Term \\
\hline Lang & wln & $377 \ddagger 1$ & Walloon & MARC Language Term \\
\hline Lang & wol & $377 \ddagger 1$ & Wolof & MARC Language Term \\
\hline Lang & xal & $377 \ddagger 1$ & Oirat & MARC Language Term \\
\hline Lang & xho & $377 \ddagger 1$ & Xhosa & MARC Language Term \\
\hline Lang & yao & $377 \ddagger 1$ & Yao (Africa) & MARC Language Term \\
\hline Lang & yap & $377 \ddagger 1$ & Yapese & MARC Language Term \\
\hline Lang & yid & $377 \ddagger 1$ & Yiddish & MARC Language Term \\
\hline Lang & yor & $377 \ddagger 1$ & Yoruba & MARC Language Term \\
\hline Lang & ypk & $377 \ddagger 1$ & Yupik languages & MARC Language Term \\
\hline Lang & zap & $377 \ddagger 1$ & Zapotec & MARC Language Term \\
\hline Lang & $\mathrm{zbl}$ & $377 \ddagger 1$ & Blissymbolics & MARC Language Term \\
\hline Lang & zen & $377 \ddagger 1$ & Zenaga & MARC Language Term \\
\hline Lang & zha & $377 \ddagger 1$ & Zhuang & MARC Language Term \\
\hline Lang & znd & $377 \ddagger 1$ & Zande languages & MARC Language Term \\
\hline Lang & zul & $377 \ddagger 1$ & Zulu & MARC Language Term \\
\hline Lang & zun & $377 \ddagger 1$ & Zuni & MARC Language Term \\
\hline Lang & $z x x$ & $377 \ddagger 1$ & No linguistic content & MARC Language Term \\
\hline Lang & zza & $377 \ddagger 1$ & Zaza & MARC Language Term \\
\hline LitF & 0 & & & No equivalent value \\
\hline LitF & 1 & $655 \neq a$ & Fiction & Genre Term (LCGFT) \\
\hline LitF & $d$ & $655 \neq a$ & Drama & Genre Term (LCGFT) \\
\hline LitF & $\mathrm{e}$ & $655 \ddagger a$ & Essays & Genre Term (LCGFT) \\
\hline LitF & $f$ & $655 \neq a$ & Novels & Genre Term (LCGFT) \\
\hline LitF & $\mathrm{h}$ & $655 \neq a$ & Humor & Genre Term (LCGFT) \\
\hline LitF & $\mathrm{i}$ & $655 \neq a$ & Personal correspondence & Genre Term (LCGFT) \\
\hline LitF & j & $655 \ddagger a$ & Short stories & Genre Term (LCGFT) \\
\hline LitF & $\mathrm{m}$ & & & No equivalent value \\
\hline LitF & $\mathrm{p}$ & $655 \neq a$ & Poetry & Genre Term (LCGFT) \\
\hline LitF & s & $655 \neq a$ & Speeches & Genre Term (LCGFT) \\
\hline LitF & $\mathrm{u}$ & & & No equivalent value \\
\hline LTxt & <blank> & & & No equivalent value \\
\hline LTxt & $\mathrm{a}$ & $655 \neq a$ & Autobiographies & Genre Term (LCGFT); Merged value \\
\hline LTxt & $\mathrm{b}$ & $655 \ddagger a$ & Biographies & Genre Term (LCGFT) \\
\hline LTxt & c & $655 \neq a$ & Conference papers and proceedings & Genre Term (LCGFT) \\
\hline LTxt & $d$ & $655 \ddagger a$ & Drama & Genre Term (LCGFT) \\
\hline LTxt & $\mathrm{e}$ & $655 \neq a$ & Essays & Genre Term (LCGFT) \\
\hline LTxt & $f$ & $655 \neq a$ & Fiction & Genre Term (LCGFT) \\
\hline LTxt & g & $655 \neq a$ & Radio field reports & Genre Term (LCGFT) \\
\hline LTxt & $\mathrm{h}$ & & & No equivalent value \\
\hline LTxt & $\mathrm{i}$ & $655 \neq a$ & Programmed instructional materials & Genre Term (LCGFT); Merged value \\
\hline LTxt & j & $655 \neq a$ & Programmed instructional materials & Genre Term (LCGFT); Merged value \\
\hline LTxt & $\mathrm{k}$ & $655 \neq a$ & Humor & Genre Term (LCGFT) \\
\hline LTxt & 1 & $655 \ddagger a$ & Speeches & Genre Term (LCGFT) \\
\hline LTxt & $\mathrm{m}$ & $655 \neq a$ & Autobiographies & Genre Term (LCGFT); Merged value \\
\hline LTxt & $\mathrm{n}$ & & & No equivalent value \\
\hline
\end{tabular}


Conversion Table for the Fixed Field

\begin{tabular}{|c|c|c|c|c|}
\hline \multicolumn{2}{|c|}{ Fixed Field Value } & \multicolumn{2}{|c|}{ Variable Field Value } & \multirow{2}{*}{$\begin{array}{l}\text { Notes } \\
\text { Genre Term (LCGFT) }\end{array}$} \\
\hline LTxt & 0 & $655 \neq a$ & Folk tales & \\
\hline LTxt & $\mathrm{p}$ & $655 \neq a$ & Poetry & Genre Term (LCGFT) \\
\hline LTxt & r & $655 \neq a$ & Radio rehearsals & Genre Term (LCGFT) \\
\hline LTxt & $\mathrm{s}$ & 655 ‡a & Sound recordings & Genre Term (LCGFT) \\
\hline LTxt & $\mathrm{t}$ & $655 \neq a$ & Interviews & Genre Term (LCGFT) \\
\hline LTxt & $z$ & & & No equivalent value \\
\hline Mrec & $<$ blank $>$ & & & Administrative metadata \\
\hline Mrec & $d$ & & & Administrative metadata \\
\hline Mrec & 0 & & & Administrative metadata \\
\hline Mrec & r & & & Administrative metadata \\
\hline Mrec & $\mathrm{s}$ & & & Administrative metadata \\
\hline Mrec & u & & & Administrative metadata \\
\hline Mrec & $x$ & & & Administrative metadata \\
\hline OCLC & & & & Administrative metadata \\
\hline Orig & $<$ blank> & & & No equivalent element \\
\hline Orig & a & & & No equivalent element \\
\hline Orig & $b$ & & & No equivalent element \\
\hline Orig & c & & & No equivalent element \\
\hline Orig & $d$ & & & No equivalent element \\
\hline Orig & e & & & No equivalent element \\
\hline Orig & $f$ & & & No equivalent element \\
\hline Orig & 0 & & & No equivalent element \\
\hline Orig & $q$ & & & No equivalent element \\
\hline Orig & $s$ & & & No equivalent element \\
\hline Part & <blank> & & & No equivalent element \\
\hline Part & $d$ & & & No equivalent element \\
\hline Part & $\mathrm{e}$ & & & No equivalent element \\
\hline Part & $f$ & & & No equivalent element \\
\hline Part & $\mathrm{n}$ & & & No equivalent element \\
\hline Part & $\mathrm{u}$ & & & No equivalent element \\
\hline Proj & aa & $255 \neq b$ & Aitoff & Proposed RDA value \\
\hline Proj & $a b$ & $255 \neq b$ & Gnomic & Proposed RDA value \\
\hline Proj & $\mathrm{ac}$ & $255 \ddagger b$ & Lambert's azimuthal equal area & Proposed RDA value \\
\hline Proj & ad & $255 \ddagger b$ & Orthographic & Proposed RDA value \\
\hline Proj & ae & $255 \neq b$ & Azimuthal equidistant & Proposed RDA value \\
\hline Proj & af & $255 \ddagger b$ & Stereographic & Proposed RDA value \\
\hline Proj & ag & $255 \ddagger b$ & General vertical near-sided & Proposed RDA value \\
\hline Proj & am & $255 \ddagger b$ & Modified stereographic for Alaska & Proposed RDA value \\
\hline Proj & an & $255 \neq b$ & Chamberlin trimetric & Proposed RDA value \\
\hline Proj & ap & $255 \ddagger b$ & Polar stereographic & Proposed RDA value \\
\hline Proj & $\mathrm{au}$ & $255 \neq b$ & Azimuthal, specific type unknown & Proposed RDA value \\
\hline Proj & $a z$ & $255 \ddagger b$ & Azimuthal, other & Proposed RDA value \\
\hline Proj & ba & $255 \ddagger b$ & Gall & Proposed RDA value \\
\hline Proj & $\mathrm{bb}$ & $255 \ddagger b$ & Goode's homolographic & Proposed RDA value \\
\hline Proj & $\mathrm{bc}$ & $255 \neq b$ & Lambert's cylindrical equal area & Proposed RDA value \\
\hline Proj & bd & $255 \ddagger b$ & Mercator & Proposed RDA value \\
\hline Proj & be & $255 \neq b$ & Miller & Proposed RDA value \\
\hline Proj & bf & $255 \neq b$ & Mollweide & Proposed RDA value \\
\hline
\end{tabular}


Conversion Table for the Fixed Field

\begin{tabular}{|c|c|c|c|c|}
\hline \multicolumn{2}{|c|}{ Fixed Field Value } & \multicolumn{2}{|c|}{ Variable Field Value } & \multirow{2}{*}{$\begin{array}{l}\text { Notes } \\
\text { Proposed RDA value }\end{array}$} \\
\hline Proj & bg & $255 \neq b$ & Sinusoidal & \\
\hline Proj & bh & $255 \neq b$ & Traverse Mercator & Proposed RDA value \\
\hline Proj & bi & $255 \neq b$ & Gauss-Kruger & Proposed RDA value \\
\hline Proj & bj & $255 \ddagger b$ & Equirectangular & Proposed RDA value \\
\hline Proj & bk & $255 \neq b$ & Krovak & Proposed RDA value \\
\hline Proj & bl & $255 \ddagger b$ & Cassini-Soldner & Proposed RDA value \\
\hline Proj & bo & $255 \neq b$ & Oblique Mercator & Proposed RDA value \\
\hline Proj & $\mathrm{br}$ & $255 \neq b$ & Robinson & Proposed RDA value \\
\hline Proj & bs & $255 \ddagger b$ & Space oblique Mercator & Proposed RDA value \\
\hline Proj & bu & $255 \neq b$ & Cylindrical, specific type unknown & Proposed RDA value \\
\hline Proj & bz & $255 \neq b$ & Cylindrical, other & Proposed RDA value \\
\hline Proj & $\mathrm{ca}$ & $255 \neq b$ & Alber's equal area & Proposed RDA value \\
\hline Proj & $\mathrm{cb}$ & $255 \neq b$ & Bonne & Proposed RDA value \\
\hline Proj & $\mathrm{cc}$ & $255 \neq b$ & Lambert's conformal conic & Proposed RDA value \\
\hline Proj & ce & $255 \neq b$ & Equidistant conic & Proposed RDA value \\
\hline Proj & $\mathrm{cp}$ & $255 \ddagger b$ & Polyconic & Proposed RDA value \\
\hline Proj & $\mathrm{cu}$ & $255 \neq b$ & Conic, specific type unknown & Proposed RDA value \\
\hline Proj & $\mathrm{Cz}$ & $255 \ddagger b$ & Conic, other & Proposed RDA value \\
\hline Proj & da & $255 \neq b$ & Armadillo & Proposed RDA value \\
\hline Proj & $\mathrm{db}$ & $255 \neq b$ & Butterfly & Proposed RDA value \\
\hline Proj & dc & $255 \neq b$ & Eckert & Proposed RDA value \\
\hline Proj & $d d$ & $255 \neq b$ & Goode's homolosine & Proposed RDA value \\
\hline Proj & de & $255 \neq b$ & Miller's bipolar oblique conformal conic & Proposed RDA value \\
\hline Proj & df & $255 \neq b$ & Van Der Grinten & Proposed RDA value \\
\hline Proj & dg & $255 \neq b$ & Dymaxion & Proposed RDA value \\
\hline Proj & $\mathrm{dh}$ & $255 \neq b$ & Cordiform & Proposed RDA value \\
\hline Proj & dl & $255 \neq b$ & Lambert conformal & Proposed RDA value \\
\hline Proj & $\mathrm{zz}$ & & & No equivalent value \\
\hline Rec stat & $\mathrm{a}$ & & & Administrative metadata \\
\hline Rec stat & c & & & Administrative metadata \\
\hline Rec stat & $\mathrm{n}$ & & & Administrative metadata \\
\hline Rec stat & $\mathrm{p}$ & & & Administrative metadata \\
\hline Regl & $\cdot$ & & & No equivalent value \\
\hline Regl & $\mathrm{n}$ & $515 \neq a$ & Normalized irregular & Proposed RDA value \\
\hline Regl & r & $515 \neq a$ & Regular & Proposed RDA value \\
\hline Regl & $\mathrm{u}$ & & & No equivalent value \\
\hline Regl & $x$ & $515 \neq a$ & Completely irregular & Proposed RDA value \\
\hline Relf & $<$ blank $>$ & & & No equivalent element \\
\hline Relf & $\mathrm{a}$ & & & No equivalent element \\
\hline Relf & $\mathrm{b}$ & & & No equivalent element \\
\hline Relf & c & & & No equivalent element \\
\hline Relf & $d$ & & & No equivalent element \\
\hline Relf & $\mathrm{e}$ & & & No equivalent element \\
\hline Relf & $f$ & & & No equivalent element \\
\hline Relf & $\mathrm{g}$ & & & No equivalent element \\
\hline Relf & $\mathrm{i}$ & & & No equivalent element \\
\hline Relf & j & & & No equivalent element \\
\hline Relf & $\mathrm{k}$ & & & No equivalent element \\
\hline
\end{tabular}


Conversion Table for the Fixed Field

\begin{tabular}{|c|c|c|c|c|}
\hline \multicolumn{2}{|c|}{ Fixed Field Value } & \multicolumn{2}{|c|}{ Variable Field Value } & \multirow{2}{*}{$\begin{array}{l}\text { Notes } \\
\text { No equivalent element }\end{array}$} \\
\hline Relf & $\mathrm{m}$ & & & \\
\hline$\underline{\text { Relf }}$ & $\mathrm{z}$ & & & No equivalent element \\
\hline Replaced & <yyymmc & ddhhmmss.f> & & Administrative metadata \\
\hline $\mathrm{SpFm}$ & $<$ blank> & & & No equivalent value \\
\hline $\mathrm{SpFm}$ & $\mathrm{e}$ & $340 \neq d$ & manuscript & \\
\hline SpFm & j & $300 \neq f$ & postcard & \\
\hline $\mathrm{SpFm}$ & $\mathrm{k}$ & $300 \neq f$ & calendar & Proposed RDA value \\
\hline $\mathrm{SpFm}$ & I & $300 \neq f$ & jigsaw puzzle & \\
\hline $\mathrm{SpFm}$ & $\mathrm{n}$ & $300 \ddagger f$ & game & \\
\hline $\mathrm{SpFm}$ & 0 & $300 \neq f$ & wall map & Proposed RDA value \\
\hline $\mathrm{SpFm}$ & $\mathrm{p}$ & $300 \neq f$ & playing cards & \\
\hline $\mathrm{SpFm}$ & $r$ & $300 \neq f$ & volume (loose-leaf) & \\
\hline $\mathrm{SpFm}$ & $z$ & & & No equivalent value \\
\hline Srce & <blank> & & & Administrative metadata \\
\hline Srce & c & & & Administrative metadata \\
\hline Srce & $\mathrm{d}$ & & & Administrative metadata \\
\hline$\overline{\text { SrTp }}$ & $<$ blank $>$ & & & No equivalent element \\
\hline SrTp & $d$ & & & No equivalent element \\
\hline SrTp & I & & & No equivalent element \\
\hline SrTp & $\mathrm{m}$ & & & No equivalent element \\
\hline SrTp & $\mathrm{n}$ & & & No equivalent element \\
\hline SrTp & $\mathrm{p}$ & & & No equivalent element \\
\hline SrTp & w & & & No equivalent element \\
\hline$\overline{S / L}$ & 0 & & & Administrative metadata \\
\hline$S / L$ & 1 & & & Administrative metadata \\
\hline $\mathrm{S} / \mathrm{L}$ & 2 & & & Administrative metadata \\
\hline Tech & $\mathrm{a}$ & $655 \neq a$ & $\begin{array}{l}\text { Animated films / Animated television } \\
\text { programs }\end{array}$ & Genre Term (LCGFT); Split values \\
\hline Tech & c & $655 \neq a$ & $\begin{array}{l}\text { Live-action/animation films / Live- } \\
\text { action/animation television programs }\end{array}$ & Genre Term (LCGFT); Split values \\
\hline Tech & I & & & No equivalent value \\
\hline Tech & $\mathrm{n}$ & & & No equivalent value \\
\hline Tech & $\mathrm{u}$ & & & No equivalent value \\
\hline Tech & $\mathrm{z}$ & & & No equivalent value \\
\hline Time & 000 & & & No equivalent value \\
\hline Time & $<\mathrm{mmm}>$ & $306 \neq a$ & & Mathematical operations required \\
\hline Time & --- & & & No equivalent value \\
\hline Time & $\mathrm{nnn}$ & & & No equivalent value \\
\hline Tmat & $a$ & & & No equivalent value \\
\hline Tmat & $\mathrm{b}$ & & & No equivalent value \\
\hline Tmat & c & & & No equivalent value \\
\hline Tmat & $d$ & $300 \neq f$ & diorama & \\
\hline Tmat & $f$ & $338 \neq a$ & filmstrip & Do not migrate \\
\hline Tmat & $\mathrm{g}$ & $300 \neq f$ & game & \\
\hline Tmat & $\mathrm{i}$ & $300 \ddagger f$ & picture & \\
\hline Tmat & $\mathrm{k}$ & & & No equivalent value \\
\hline Tmat & I & $300 \ddagger f$ & technical drawing & \\
\hline Tmat & $\mathrm{m}$ & & & No equivalent value \\
\hline
\end{tabular}


Conversion Table for the Fixed Field

\begin{tabular}{|c|c|c|c|c|}
\hline \multicolumn{2}{|c|}{ Fixed Field Value } & \multicolumn{2}{|c|}{ Variable Field Value } & \multirow[t]{2}{*}{ Notes } \\
\hline Tmat & $\mathrm{n}$ & $300 \neq f$ & chart & \\
\hline Tmat & 0 & $300 \neq f$ & flash card & \\
\hline Tmat & $\mathrm{p}$ & $338 \neq a$ & microscope slide & Do not migrate \\
\hline Tmat & $q$ & $300 \neq f$ & model & \\
\hline Tmat & $r$ & & & No equivalent value \\
\hline Tmat & s & $338 \neq a$ & slide & Do not migrate \\
\hline Tmat & $\mathrm{t}$ & $338 \neq a$ & overhead transparency & Do not migrate \\
\hline Tmat & v & & & No equivalent value \\
\hline Tmat & w & $300 \neq f$ & toy & \\
\hline Tmat & $z$ & & & No equivalent value \\
\hline $\operatorname{TrAr}$ & \multicolumn{2}{|l|}{$<$ blank $>$} & & No equivalent element \\
\hline $\operatorname{TrAr}$ & \multicolumn{2}{|l|}{$\mathrm{a}$} & & No equivalent element \\
\hline $\operatorname{TrAr}$ & \multicolumn{2}{|l|}{$\mathrm{b}$} & & No equivalent element \\
\hline $\operatorname{TrAr}$ & \multicolumn{2}{|l|}{ c } & & No equivalent element \\
\hline $\operatorname{TrAr}$ & \multicolumn{2}{|l|}{$\mathrm{n}$} & & No equivalent element \\
\hline $\operatorname{TrAr}$ & \multicolumn{2}{|l|}{$\mathrm{u}$} & & No equivalent element \\
\hline Type & $a$ & $336 \neq a$ & text & \\
\hline Type & c & $336 \neq a$ & notated music & \\
\hline Type & $d$ & $336 \neq a$ & notated music & Merged value \\
\hline Type & \multicolumn{2}{|l|}{$\mathrm{e}$} & & No equivalent value \\
\hline Type & \multicolumn{2}{|l|}{$f$} & & \multirow[t]{2}{*}{ No equivalent value } \\
\hline Type & g & $337 \neq a$ & projected & \\
\hline Type & $\mathrm{i}$ & $336 \neq a$ & sounds/spoken word & Split values \\
\hline Type & j & $336 \neq a$ & performed music & \\
\hline Type & $\mathrm{k}$ & $336 \neq a$ & still image & \\
\hline Type & \multicolumn{2}{|c|}{ and... $337 \ddagger a$} & unmediated & \\
\hline Type & $\mathrm{m}$ & $336 \neq a$ & computer dataset/computer program & Split values \\
\hline Type & 0 & & & No equivalent value \\
\hline Type & $\mathrm{p}$ & & & No equivalent value \\
\hline Type & r & $336 \neq a$ & three-dimensional form & \\
\hline Type & $\mathrm{t}$ & $336 \neq a$ & text & Merged value \\
\hline
\end{tabular}

